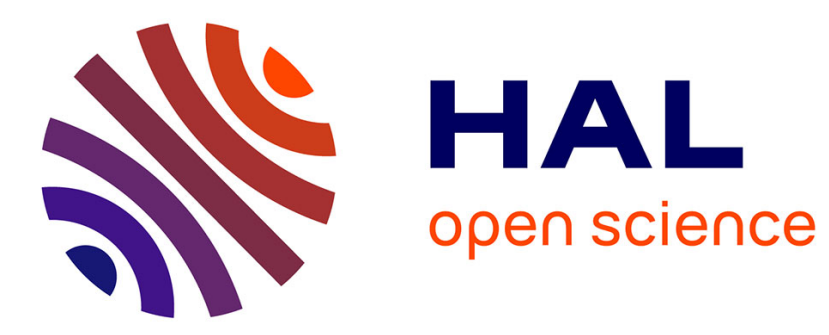

\title{
PHOTOIONISATION OF ATOMS AND IONS : AN EXTENDED BIBLIOGRAPHY FOR 1986-1990
}

\author{
M. Le Dourneuf
}

\section{To cite this version:}

M. Le Dourneuf. PHOTOIONISATION OF ATOMS AND IONS: AN EXTENDED BIBLIOGRAPHY FOR 1986-1990. Journal de Physique IV Proceedings, 1991, 01 (C1), pp.C1-227-C1-285. 10.1051/jp4:1991125 . jpa-00249763

\section{HAL Id: jpa-00249763 https://hal.science/jpa-00249763}

Submitted on 1 Jan 1991

HAL is a multi-disciplinary open access archive for the deposit and dissemination of scientific research documents, whether they are published or not. The documents may come from teaching and research institutions in France or abroad, or from public or private research centers.
L'archive ouverte pluridisciplinaire HAL, est destinée au dépôt et à la diffusion de documents scientifiques de niveau recherche, publiés ou non, émanant des établissements d'enseignement et de recherche français ou étrangers, des laboratoires publics ou privés. 


\title{
PHOTOIONISATION OF ATOMS AND IONS : AN EXTENDED BIBLIOGRAPHY FOR 1986-1990
}

\author{
M. LE DOURNEUF \\ Laboratoire de Théorie des Processus Atomiques et Moléculaires (UPR 261 \\ du CNRS), Departement "Atomes et Molécules an Astrophysique", \\ observatolre de Paris, F-92195 Meudon, France
}

\begin{abstract}
RESUME : Une liste de plus de 900 publications parues en 1986-90 sur les transitions lié-libre induites par impact photonique dans les systèmes atomiques est présentée. Ce travail étend la bibliographie 1970-87 de Butler et al (1988) sur la photoionisation des atomes et des ions dans deux directions. D'une part, elle élargit le domaine d'investigation systematique à l'ionisation simple et multiple, directe et résonnante, impliquant des couches externes ou internes, induite par un seul ou un grand nombre de photons, en présence ou non de champs statiques externes. D'autre part, elle affine l'indexation des références obtenues qui sont classées simultanément par espèce, type de processus et de travail.
\end{abstract}

\begin{abstract}
A list of over 900 references published during the period 1986-90 on bound-free transitions induced by photon-impact in atomic systems is presented. This work extends the 1970-87 bibliography of Butler et al (1988) on the photoionisation of atoms and ions in two directions. On one hand, it enlarges the domain of systematic investigation to simple and multiple ionisation, direct and resonant, implying outer and inner shells, induced by a single or a large number of photons, with or without the presence of external static fields. On the other hand, it refines the indexation of the selected references by species, type of process and of work.
\end{abstract}

\section{PRESENTATION OF THE BIBLIOGRAPHY}

The study of photon interactions with atoms and molecules is a rapidly expanding field of research, due to the development of versatile experimental tools (synchrotron radiation, lasers) and powerful computational techniques ( $R$-matrix methods, Many Body Perturbation Theory, Random Phase Approximation with Exchange, Local Density Functional Approximation, ...), which allow to explore species and processes of increasing complexity.

It becomes timely to gather the outcome of such studies in order to make them available to related research fields, such as the modelling of radiative transfer in plasmas. The International Opacity Project on the ab initio calculation of the stellar envelope opacities (Seaton, 1988 and many contributions of the present issue) is such an integrated program, which requires the collaboration of a large number of researchers to produce radiative data, to evaluate them and introduce them in adequate equations of state in order to model the evolution of the stellar envelopes. This astrophysical project has largely promoted the activity of the International Atomic Data Workshops (Daresbury 1985, Oxford 1987, Meudon 1989), in the framework of which the present series of bibliographies on the photoionisation of atoms and ions has been undertaken (Le Dourneuf \& Zeippen 1986; Butler et al 1988, later on refered as BLZ).

This third bibliography aims at providing an extended selection of the data published during the $1986-90$ period for all types of bound-free transitions induced by one or more photons in atomic systems. Because of an increasing activity in several areas (autoionizing states, Auger spectra, multiple ionization, multiphoton processes, photoionisation in the presence of static fields), an attempt has therefore been made to fill some gaps in our previous list for 1986-87. Note however that the references of 1986 and 1987 which were already included in our previous bibliography (BLZ) have been discarded from the present list, so that the reader should systematically refer to BLZ to get a complete list for these two years. The compilation is based on a systematic investigation of the Physical Abstract Section on "Photon interaction with atoms", in parallel to that of the many journals more rapidly available at the Observatory of 
Paris (as mentioned in section 2). In addition, the analysis of the references has been deepened. The list of references (section 3) is still presented in alphabetical order with the full titles. But, concise comments on three topics are added. The first one concerns the type of publication, qualified as a review (R), mainly experimental (E), theoretical (T), or a mixture of both $(\operatorname{Exp} \&$ theory $=M)$. The second one qualifies the process under study by the association of up to three keys : OU for transitions involving outer shells, IN for those involving inner shells (inner valence shells being sometimes qualified by the associaion of both keys), AI when emphasis is put on autoionizing states and resonant processes, MI when multiple ionization is investigated, SF for photoionization in the presence of static fields, FP for processes induced by a few photons, MP for processes involving many photons. The third comment concerns the species for which specific data is given. The index based on this analysis and presented in section 4 can therefore be very detailed : it is presented by species gathered in isoelectronic or isonuclear sequences, and for each species according to the keys classifying the processes and the type od data contained in each reference. The bibliography is stored in a database created using the R:BASE system $V$ by MICRORIM, which facilitates regular updates and allows various modes of selection and presentation to be made.

This bibliography cannot be guaranteed as exhaustive or bugless, but every effort has been made to include a maximum of relevant references and to check them. In particular, efforts have been made to include as many references published in 1990 as possible before the printing of the Proceedings. Because of the difficulties specific to compilations and classifications, the present work should be considered as complementary to similar efforts made, for example, in the International Bulletin on Atomic and Molecular Data For Fusion edited by J.J. Smith for the International Atomic Energy Agency and in the Multiphoton Bibliography edited by S.J. Smith, J.H. Eberly and J.W. Gallagher for NIST.

\section{References}

K. Butler, M. Le Dourneuf and C.J. Zeippen (1988) refered as BLZ in the text. Second Atomic Data Workshop : Assessment of data for photo-ionization and photo-excitation and for electron impact excitation of atomic ions. (Oxford, August 1987) Proceedings ed. by W. Eissner \& A.E. Kingston (Daresbury, 1988) p. 20-82. "Photoionisation of atoms and ions: 1970-1987. A bibliography".

M. Le Dourneuf and C.J. Zeippen (1986). First Atomic Data Workshop : Low energy collision theory techniques for atomic excitation and radiative data. (Daresbury, March 1985) Proceedings ed. by W.B. Eissner (Daresbury report DL/SCI/R24 (Theory), 1986) p. 134-148. "Photoionisation of atoms and ions : a bibliography".

M.J. Seaton (1988), in "Electronic and Atomic Collisions", Invited Lectures of the XVth ICPEAC (Brighton 1987) ed. By H.B. Gilbody, W.R. Newell, F.H. Read and A.C.H. Smith (North Holland, 1988) p. 41-56. "Atomic physics and stellar opacities"

J.J. Smith, ed. International Bulletin on Atomic and Molecular Data for Fusion, published periodically by the International Atomic Energy Agency, Vienna.

S.J. Smith, J.H. Eberly and J.W. Gallagher. ed. Multiphoton bibliography: 1987-88. NBS-LP92, Suppl. 6, edited by the Universities of Colorado and Rochester (june 1990) and previous issues.

\section{Acknowledgements}

The author wishes to thank her two secretaries, Marie-Laure Rousseau and Karine Le Moine, for their contributions to the storage of the bibliography in the local database and her colleague, Claude Zeippen, for his advices and encouragements to finalize the presentation of the bibliography.

\section{KEYS TO JOURNAL ABBREVIATIONS}

The 909 references which are analyzed in the following bibliography are extracted from about 80 different journals or conference proceedings. The abbreviations of their titles used in the following list of papers are given below, together with a label $\left({ }^{*}\right)$ to indicate those for which the author had easy local access. While most of the 750 corresponding references have been checked individually, the indexing of the remaining ones, which could not be accessed directly, is based exclusively on Physical Abstracts, and may be less accurate. 


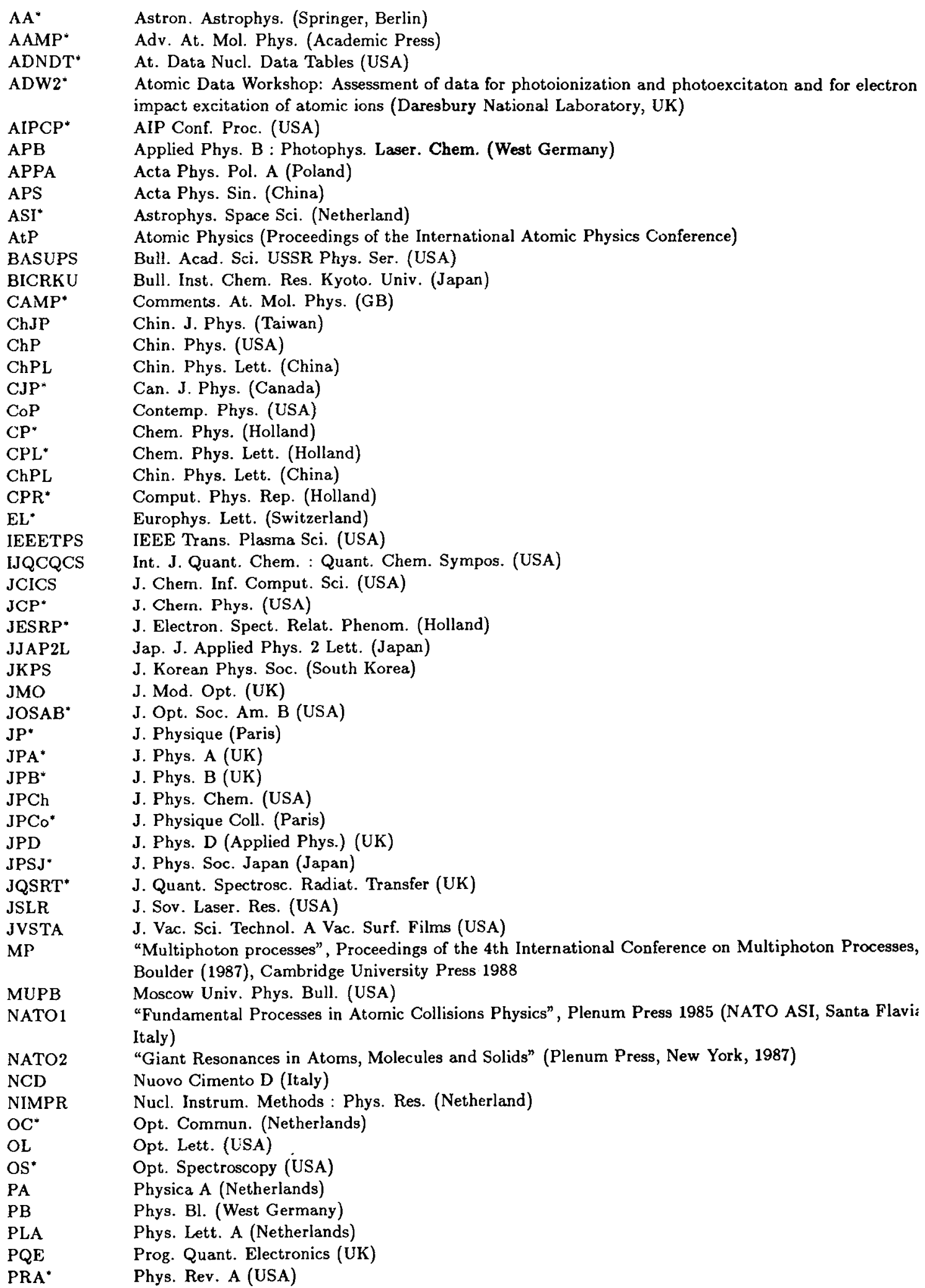




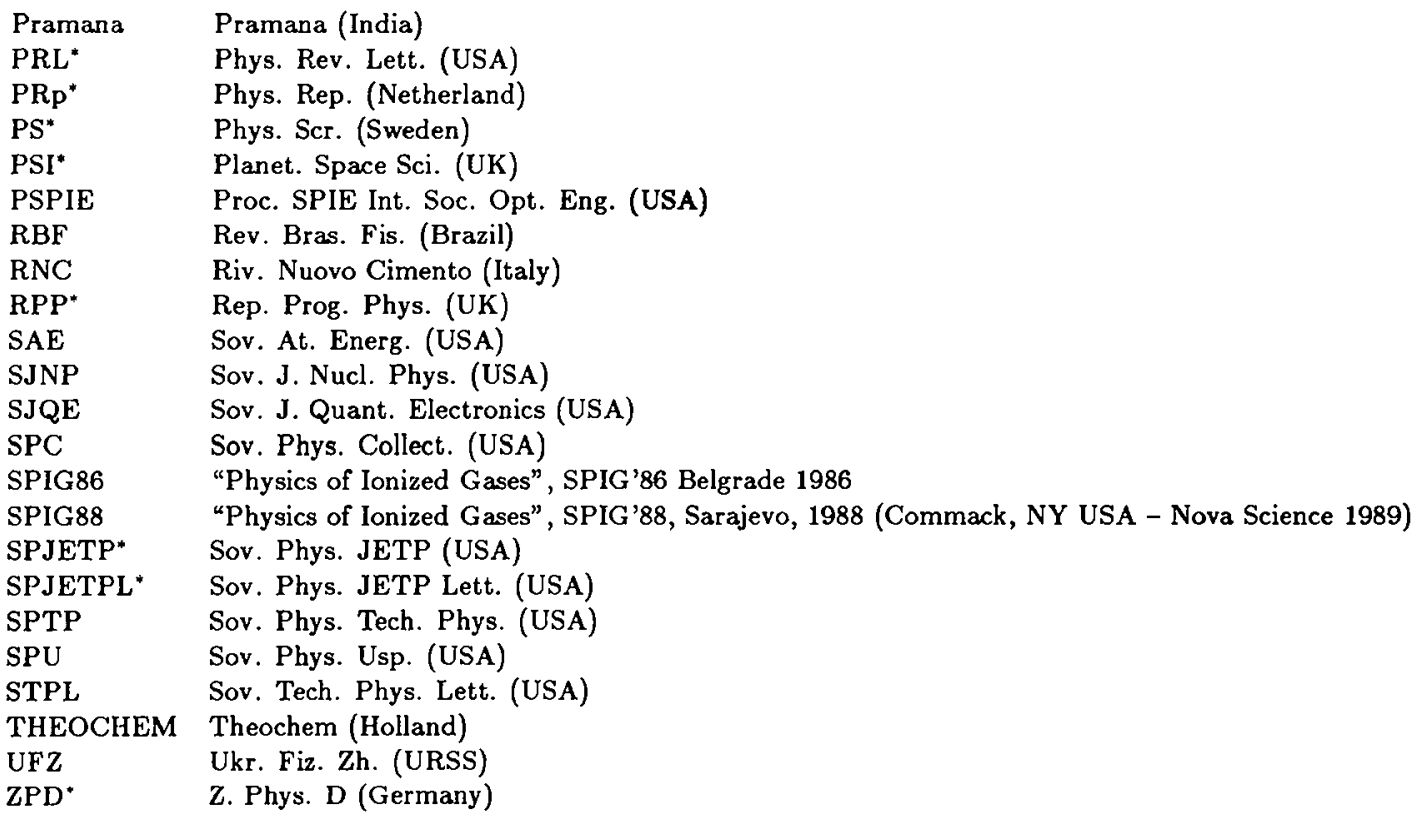

\section{BIBLIOGRAPHY}

In the following list, ordered alphabetically and chronologically, the full references are followed by a few com. ments, indicating the major philosophy of the publication (Theory, Experiment, Review), up to three keys caracterizing the processes under study (OU an/or IN specifying processes involving mainly outer and/or inner shells -inner valence shells being sometimes indexed by both-, AI, MI, SF, FP. MP indicating special emphasis respectively on autoionizing states, multiple ionization, presence of external static fields, processes involving a few or many -large or unspecified-photons) and the list of species on which special data is given (General means that no specific species is involved or could be identified because the author of the bibliography has no easy access to the reference). In some cases this indexation is obvious from the title, in other cases it gives additional information, and in all cases it indicates the keys which have been used for the index in section 4 , so that the reader can easily take care of any omission or mistake in the analysis of the references.

Note that, for years 1986 and 1987, the present bibliography gives only the references which were not included in BLZ. The reader should therefore refers to this previous bibliography to get a complete bibliography for these twc years.

[1] M.Y. Adam, J. Hellner, G. Dujardin, A. Svensson, P. Martin and F. Combet Farnoux. Single and double photoionisation of atomic silver between 43.5 and 15.5nm. JPB, 22:2141-50, 1989. Exp \& theory - Keys : or MI - Species : Ag.

[2] N.V. Afanaseva and V.O. Kozlov. Absorption cross section of atomic zinc beyond the frst ionization boundary. OS, 63:8-11, 1988. Exp \& theory - Keys : DU - Species : Zn.

[3] P. Agostini, A. Antonetti, P. Breger, M. Crance, A. Migus, H.G. Muller and G. Petite. Resonant multiphoton ionisation of xenon with high-intensity femtosecond pulses. JPB, 22:1971-7, 1989. Exp \& theory - Keys : MP Species: Xe.

[4] P. Agostini, P. Breger, A. L'Huillier, H.G. Muller, G. Petite, A. Antonetti, and A. Migus. Giant Stark shifts in multiphoton ionization. PRL, 63:2208-11, 1989. Exp \& theory - Keys : MP - Species : Xe.

[5] P. Agostini, P. Breger, A. L'Huillier, H.G. Muller, G. Petite, A. Antonetti, and A. Migus. Resonant multiphoton ionization with intense femtosecond pulses. APPA, A78:17-31, 1990. Exp. review - Keys : MP - Species: Xe. 
[6] P. Agostini and G. Petite. Photoelectric effect under strong irradiation. CoP, 29:57-77, 1988. Exp. review Keys : MP - General.

[7] H. Aksela and S. Aksela. Recent developments in Auger spectroscopy of free atoms. JPCo, 48-C9:565-77, 1987. Th. review - Keys : IN - Species : $\mathrm{Ne}, \mathrm{Ar}, \mathrm{Ni}, \mathrm{Kr}, \mathrm{Pd}, \mathrm{Ag}, \mathrm{Cd}$.

[8] H. Aksela, S. Aksela, R. Lakanen, J. Tulkki and T. Aberg. Initial- and final-state correlation in the valence-shell Auger spectrum of Rb. PRA, 42:5193-5200, 1990. Exp \& theory - Key; : IN - Species : Rb.

[9] H. Aksela, S. Aksela, H. Pulkkinen and A. Yagishita. Shake processez in the Auger decay of resonantly excited $3 d^{9} 4 s^{2} 4 p^{6} n p$ states of Kr. PRA, 40:6275-80, 1989. Exp \& theory - Keys : IN - Species : Kr.

[10] H. Aksela, S. Aksela, H. Pulkkinen, A. Kivimaki and O.P. Sairanen. Shake processes in Auger decay of resonantly excited states of rare gases. PS, 41:425-8, 1990. Exp \& theory - Keys : IN - Species : Ne, Ar, Kr, Xe.

[11] H. Aksela, S. Aksela, J. Tulkki, T. Åberg, G.M. Bancroft and K.H. Tan. Auger emission from the resonantly excited $1 s^{1} 2 s^{2} 2 p^{6} 3 p$ state of Ne. PRA, 39:3401, 1989. Exp \& theory - Keys : IN - Species : Ne.

[12] H. Aksela, R. Lakanen, S. Aksela and O. Sairanen. Resonance Auger spectra of free Rb atoms. PRA, 38:3395-9, 1988. Exp \& theory - Keys : IN - Species : Rb.

[13] G. Alber and P. Zoller. Near-threshold excitation of Rydberg series by strong laser fields. PRA, 37:377-89, 1987. Theory - Keys : MP - General.

[14] G. Alber and P. Zoller. Laser excited electronic wave packets in Rydberg atoms. PB, 45:477-8, 1989. Theory Keys : MP - Species : Rb.

[15] V.N. Aleinikov, V.G. Eremin, G.L. Klimchitskaya, I.N. Polushkin, Y.V. Rostovtsev and V.V. Yazenkov. Structure of quasi-energy spectrum of hydrogen in a microwave field ; theory and experiment. SPJETP, 67:908-14, 1988. Exp \& theory - Keys : MP - Species : H.

[16] V.N. Aleinikov and G.L. Klimchitskaya. Intensity ratio of the first satellites of the alpha line of the Balmer series of hydrogen in microwave fields. OS, 64:172-4, 1988. Theory - Keys : MP - Species : H.

17] A. Alijah, J. Hinze and J.T. Broad. Photoionisation of hydrogen in a strong magnetic field. JPB, 23:45-60, 1990. Theory - Keys : SF - Species : H.

[18] D.T. Alimov, A. Belkovskii, M.I. Gaisak, O.I. Zatsarinnii, V.I. Lendyel, V.K. Medvedeva, D.M. Petrina and I.M. Shuba. Two-photon ionization of calcium atom. UFZ, 33:658-63, 1988. Exp \& theory - Keys : FP - Species : Ca.

[19] G.C. Allen, E.J. Baerends, P. Vernooijs, J.M. Dyke, A.M. Ellis, M. Feher and A. Morris. High temperature photoelectron spectroscopy : a study of U, UO and $\mathrm{UO}_{2}$. JCP, 89:5363-72, 1988. Exp-Keys : OU - Species : U.

[20] L. Allen and N. Melikechi. Real intermediate states and the probability of two-photon absorption. SJQE, 18:716-18, 1988. Theory - Keys : FP - Species : Na.

[21] Z. Altun. Photoionization of atomic magnesium including double-electron resonances. PRA, 40:4968-76, 1989. Theory - Keys : OU AI - Species : Mg.

[22] Z. Altun, M. Kutzner and H.P. Kelly. Photoionization of the 4d subshell of xenon. PRA, 37:4671-8, 1988. Theory - Keys : IN - Species : Xe.

[23] G. Alvarez and H.J. Silverstone. Photoionization cross-section: exact expansion over resonances and natural line shape. PRA, 42:3690-7, 1989. Theory - Keys : OU - General.

[24] M.Y. Amusia. Many-electron effects in the absorption and emission of VUV radiation by atoms and ions. PS, T31:43-55, 1990. Th. review - Keys : OU - Species : Ar, Mn, I- $, \mathrm{Xe}, \mathrm{Cs}, \mathrm{La}, \mathrm{Eu}$.

[25] M.Y. Amusia and N.B. Avdonina. Intershell interaction in excited atom and ion photoionization. ZPD, 14:191-4, 1989. Theory-Keys : OU - Species : Cs, $\mathrm{La}^{2+}$.

[26] M.Y. Amusia, L.V. Chernysheva, V.K. Ivanov and V.A. Kupchenko. Photoionization of lanthanum and its ions in the region of the "giant" resonance. ZPD, 14:215-17, 1989. Theory-Keys : OU -Species: $\mathrm{La}^{3+}, \mathrm{La}^{2+}, \mathrm{La}^{+}$, La. 
[27] M.Y. Amusia, L.V. Chernysheva, G.F. Gribakin and K.L. Tsemekhman. Single and double photoionisation in Xe and Ba above the $4 \mathrm{~d}$ threshold. JPB, 23:393-402, 1990. Theory - Keys : OU IN MI - Species: Xe, Ba.

[28] M.Y. Amusia, V.K. Dolmatov and V.M. Romanenkc. Exchange electron correlation effects in outer-level photoionisation of half-filled subshell atoms (Mn). JFB, 21:L̈1-4, 1988. Theory - Keys : oU - Species : Mn.

[29] M.Y. Amusia, G.F. Gribakin, V.K. Ivanov and L.V. Chernysheva. Many-electron correlation in negative-ion photodetachment. JPB, 23:385-91, 1990. Theory-Keys : OU - Species : $\mathrm{Na}^{-}, \mathrm{Si}^{-}, \mathrm{Ag}^{-}, \mathrm{I}^{-}$.

[30] M.Y. Amusia and V.K. Ivanov. Intershell interaction in atoms. SPU, 30:449-74, 1988. Th. review - Keys : 0U - General.

[31] M.Y. Amusia, V.K. Ivanov and V.A. Kupchenko. The photoionization of atomic Eu in the vicinity of its giant resonance. ZPD, 14:219-21, 1989. Theory-Keys : OU -- Species : Eu.

[32] M.Y. Amusia, N.M. Kabachnik, I.S. Li and I.P. Sazhina. Influence of many-electron correlations on the angular distribution and spin polarization of Auger clectrons in atoms of krypton and xenon. SPTP, 33:957-9, 1989. Theory-Keys : IN - Species : Kr, Xe.

[33] M.Y. Amusia, A.N. Kolesnikova and L.S. Li. Radiative semi-Auger satellites in X-ray spectra. SPTP, 33:268-73, 1988. Theory - Keys : IN - Species : Ne, Ar.

[34] M.Y. Amusia and M.L. Shmatov. Photoionization of inner atomic shells by thermal radiation. STPL, 14:546-7, 1989. Theory - Keys : IN - Species : Fe, Cu.

[35] G.C. Angel and J.A.R. Samson. Total photoionization cross sections of atomic oxygen from threshold to 44.3 A. PRA, 38:5578-85, 1988. Exp-Keys : DU - Species : O.

[36] H.S. Antunes-Neto, L. Davidivich, P.W. Milonni, F. Trombetta, S. Basile and G. Ferrante. Comment on 'Multiphoton-ionization transition amplitudes and the Keldysh approximation' and reply. PRA, 41:4095-6, 1990. Theory - Keys : MP - General.

[37] M. Ardehali, P.H. Mahowald and I. Lindau. The energy dependence of the 4d partial photoionization cross section in metallic $\mathrm{Ag}$ and in $\mathrm{Ag}$ atoms on $\mathrm{Si}$ at the Cooper minimum and near the 4d threshold. JESRP, 48:353-62, 1989. Exp-Keys : OU - Species : Ag.

[38] E. Arimondo, C.E. Burkhardt, F. Giammanco, L.J. Qin and A. Vellante. Multi-photon laser ionization of sodium in the 540-600 $\mathrm{nm}$ range. OC, 71:52-8, 1989. Exp - Keys : MP - Species : Na.

[39] G.B. Armen. Angle dependence of post-collision interaction Auger line shapes. PRA, 37:995-8, 1988. Theory Keys : IN - Species : Xe.

[40] G.B. Armen, B.I. Craig, F.P. Larkins and J.A. Richards. Photoionization cross-section calculations for atomic lithium. JESRP, 51:183-96, 1990. Theory - Keys : IN - Species : Li.

[41] G.B. Armen, J. Tulkki, T. Åberg and B. Crasemann. Quantum theory of post collision interaction in inner-shell photoionization. JPCo, 48-C9:479-82, 1987. Th. review - Keys : IN - Species : Xe.

[42] U. Arp, G. Materlik, M. Richter and B. Sonntag. $Z$-dependent differences between experimental and theoretical 2p-core-hole widths of atomic rare earths. JPB, 23:L811-6, 1990. Exp-Keys : IN AI - Species : Xe, Ce, Sm, Gd, Er, Yb.

[43] G.P. Arrighini, C. Guidotti and N. Durante. Photoionization by a variational approach : a simple application to the hydrogen atom. CPL, 141:361-5, 1987. Theory - Keys : OU - Species : H.

[44] G.P. Arrighini, C. Guidotti and N. Durante. Photodetachment cross section of analytically tractable systems : the extended asymptotic model revisited. THEOCHEM, 43:369-74, 1988. Theory - Keys : OU - Species : $\mathrm{H}^{-}$.

[45] G.P. Arrighini, C. Guidotti and N. Durante. A statistical quasi-classical approach to photoionization. CPL, 159:56-60, 1989. Theory-Keys : OU - Species : H.

[46] G.P. Arrighini, C. Guidotti and U.T. Lamanna. Two-photon detachment cross section of atoms in the extended asymptotic model. NCD, 12D:1079-92, 1990. Theory - Keys : FP - Species : $\mathrm{H}^{-}$.

[47] N. Asida, D. Albeck and M. Rosenbluh. Absolute photoionisation cross section of the $11^{3} \mathrm{D}$ state of ${ }^{4} \mathrm{He}$. JPB, 20:L121-5, 1986. Exp - Keys : OU - Species : He. 
[48] G. Aspromallis and C.A. Nicolaides. Relativistic and Coulomb autoionisation in low-lying excited states of fluorine. JPB, 19:L13-18, 1986. Theory-Keys : AI - Species : F.

[49] J. Auerhammer, H. Genz, A. Kumar and A. Richter. Two-electron-one-photon transition in aluminium following double-K-shell ionization. PRA, 38:688-93, 1988. Exp-Keys : IN MI-Species : Al.

[50] N.B. Avdonina and M.Y. Amusia. Photoionization of excited states of the cesium isoelectronic series [and longrange Coulomb potential influence]. OS, 66:578-81, 1989. Theory-Keys : ou - Species : Cs seq (Cs, $\mathrm{Ba}^{+}$, $\left.\mathrm{La}^{2+}\right)$.

[51] M. Aymar. Eigenchannel $R$-matrix calculation of the $J=1$ odd-parity spectrum of barium. JPB, 23:2697-716, 1990. Theory - Keys : OU - Species : Ba.

[52] M. Aymar and J.M. Lecomte. $R$-matrix calculation of photoionisation from Rydberg states in strontium. JPB, 22:223-45, 1989. Theory - Keys : oU - Species : Sr.

[53] M. Aymar, E. Luc-Koenig and S. Watanabe. $R$-matrix calculation of eigenchannel multichannel quantum defect parameters for strontium. JPB, 20:4325-45, 1987. Theory - Keys : OU - Species : Sr.

[54] A.P. Babichev, E.B. Gel'man, I.S. Grigor'ev, O.V. Latyshev, A.F. Semerok, V.A. Firsov and A.V. Chankin. Isotopically selective photoionization of calcium atoms. SJQE, 18:553-5, 1988. Exp-Keys : OU - Species : Ca.

[55] H. Bachau. Correlations in the doubly excited states of $\mathrm{O}^{6+}\left(3 \ln l^{\prime}\right)$ with $n=3,4$. JPB, 21:3547-58, 1988. Theory - Keys : AI - Species : $\mathrm{O}^{6+}$.

[56] H. Bachau and P. Lambropoulos. Theory of single and double ionization of helium through two-photon excitation of the ${ }^{1} S_{0}^{e}(1)$ doubly excited resonance. ZPD, 11:37-44, 1988. Theory-Keys : FP MI - Species : He.

[57] J. Bae, S. Kim, J. Choi, S.D. Oh and Y.S. Kim. Survey of shape resonances for photoionizing transitions. JKPS, 22:129-35, 1989. Review-Keys : ou -Species: Fe, Xe, Cs, Rn.

[58] Y.K. Bae and J.R. Peterson. Near-threshold measurements of $\mathrm{K}^{-}$two-electron photoionization cross sections. PRA, 37:3254-8, 1988. Exp-Keys : MI - Species: $\mathrm{K}^{-}$.

[59] V.G. Bagrov, G.F. Kopytov, V.B. Tlyachev and N.I. Fedosov. Polarization of relativistic photoelectrons. SPJETP, 67:1085-8, 1988. Theory - Keys : OU IN - General.

[60] S. Baier, M. Martins, B.R. Muller and P. Zimmermann. Lifetime measurements and Stark mixing of autoionizing Cu I-states. ZPD, 10:445-9, 1988. Exp - Keys : AI - Species : Cu.

[61] S. Baier, M. Martins, B.R. Muller, M. Schulze and P. Zimmermann. Investigation of autoionizing levels in the $\mathrm{Ag}$ I spectrum populated by a combination of discharge and laser excitation. JPB, 23:3095-104, 1990. ExpKeys : AI FP - Species: Ag.

[62] M.A. Baig, 1. Ahmed and J.P. Connerade. Autoionisation resonances in the 5s-subshell excitation spectrum of indium. JPB, 21:35-46, 1988. Exp-Keys : OU AI - Species : In.

[63] M.A. Baig, J.P. Connerade and M. Rafi. A new high-resolution study of the 3d-subshell excitation spectrum of chromium. JPB, 20:L741-6, 1987. Exp - Keys : OU - Species : Cr.

[64] M.A. Baig, A. Rashid, I. Ahmad, M. Rafi, J.P. Connerade and J. Hormes. High-resolution photoabsorption study of the 3d spectrum of chromium. JPB, 23:3489-3509, 1990. Exp-Keys : OU - Species : Cr.

65] P.N. Bajaj, K.G. Manohar, B.M. Suri, K. Dasgupta, R. Talukdar, P.K. Chakraborti and P.R.K. Rao. Two colour multiphoton ionization spectroscopy of uranium from a metastable state. APB, B47:55-9, 1988. Exp-Keys : FP - Species : U.

[66] S.J. Bajic, R.N. Compton, X. Tang, A. L'Huillier and P. Lambropoulos. Multiphoton ionization photoelectron spectroscopy of xenon : experiment and theory. AIPCP, 191:466-8, 1989. Exp \& theory - Keys : FP - Species : Xe.

[67] V.V. Balashov, A.N. Grum-Grzhimailo and V. Zhadamba. Direct and resonance ionization of atomic sodium in crossed laser and synchrotron radiation beams : theory. OS, 65:315-19, 1988. Theory - Keys : FP OU - Species : $\mathrm{Na}$. 
[68] P. Baltzer and L. Karlsson. High-resolution extreme-ultraviolet spectrum of He between 35 and 55 eV. PRA, 38:2322-31, 1988. Exp - Keys : OU - Species : He.

[69] K.L. Baluja and C.J. Zeippen. The photoionisation of the $\mathrm{O}^{+}\left({ }^{4} \mathrm{~S}^{\circ}\right)$ ground state. JPB, 21:2441-54, 1988. Theory - Keys : OU - Species : $\mathrm{O}^{+}$.

[70] D.J. Bamford, W.K. Bischel, A.P. Hickman and M.J. Dyer. Measurement of two-photon absorption cross sections in atomic $O$ at $226 \mathrm{~nm}$ : single frequency versus multimode lasers. PSPIE, 912:139-44, 1989. Exp - Keys : FP - Species : O.

[71] I.M. Band, M.B. Trzhaskovskaya, D.A. Werner and D.G. Yakovlev. $K$-shell photoionization cross sections : calculations and simple fitting formulae. AA, 237:267-9, 1990. Theory - Keys : IN - Species : All $1 \leq Z \leq 26$.

[72] G. Baravian, J. Jolly, P. Persuy and G. Sultan. Resonant four-photon ionization of H atoms at $364.7 \mathrm{~nm}$. CPL, 159:361-5, 1989. Exp - Keys : FP - Species : H.

[73] J.N. Bardsley, A. Szoke and M.J. Comella. Multiphoton ionisation from a short-range potential by short-pulse lasers. JPB, 21:3899-916, 1988. Theory - Keys : MP - General.

[74] K. Bartschat and B.M. McLaughlin. Near-threshold photoionization of atomic barium from the (6s6p) ${ }^{1} \mathrm{P}_{1}^{\circ}$ sate. JPB, 23:L439-41, 1990. Theory - Keys : OU - Species : Ba.

[75] V. Bartzis. Intensity-dependent, two-photon Jaynes-Cummings model. PA, 166:347-60, 1990. Theory - Keys : MP - General.

[76] M.C. Baruch, T.F. Gallagher and D.J. Larson. Photodetachment in a microwave field. PRL, 65:1336-9, 1990. Exp - Keys : MP - Species : $\mathrm{Cl}^{-}$.

[77] M. Bashkansky, P.H. Nucksbaum and D.W. Schumacher. Asymmetries in above-threshold ionization. PRL, 60:2458-61, 1988. Exp-Keys : MP - Species : He, Kr, Xe.

[78] S. Basile, G. Ferrante and F. Trombetta. Multiphoton multichannel ionisation of hydrogen with elliptically polarised light. JPB, 21:L377-82, 1988. Theory - Keys : MP - Species : H.

[79] S. Basile, G. Ferrante and F. Trombetta. High-intensity multiphoton ionization of atoms and radiation properties. Chaotic field effects. NCD, 12D:303-22, 1990. Theory - Keys : MP - Species : H.

[80] S. Basile, F. Trombetta, G. Ferrante, R. Burlon and C. Leone. Multiphoton ionisation of hydrogen by a strong multimode field. PRA, 37:1050-2, 1988. Theory - Keys : MP - Species : H.

[81] S. Basile, F. Trombetta and G. Ferrante. Twofold symmetric angular distributions in multiphoton ionization with elliptically polarized light. PRL, 61:2435-7, 1988. Theory - Keys : MP - Species : H.

[82] S. Basile, F. Trombetta and G. Ferrante. Multiphoton ionization and collisions in strong laser fields. SJQE, 18:733-7, 1988. Theory - Keys : MP - General.

[83] D. Baudrauk. Atomic and molecular processes with short intenses laser pulses. NATO ASI Series B, vol. 171, 1987. Review - Keys : MP - General.

[84] D.R. Beck and C.A. Nicolaides. Theory of Auger energies in free atoms : application to the alkaline earths. PRA, 33:3885-90, 1986. Theory - Keys : IN AI - Species : Be, Mg, Ca.

[85] U. Becker. The $4 \mathrm{~d} \rightarrow 4 \mathrm{ff}$ giant resonances from barium through the rare-earths. NATO2, p. 473-82, 1987. Exp. review - Keys : IN - Species : Ba, Re.

[86] U. Becker. Synchrotron radiation experiments on atoms and molecules. AIPCP, 205:162-75, 1990. Exp. review - Keys : OU IN AI - Species : Li, , Ar Ne, Yb.

[87] U. Becker, H.G. Kerkhoff, M. Kupsch, B. Langer and R. Wehlitz. Photoionization of xenon with soft X-rays. JPCo, 48-C9:497-500, 1987. Exp-Keys : IN - Species : Xe.

[88] U. Becker, B. Langer, H.G. Kerkhoff, M. Kupsch, D. Szostak, R. Wehlitz, P.A. Heimann, S.H. Liu, D.W. Lindle, T.A. Ferrett and D.A. Shirley. Observation of many new argon valence satellites near threshold. PRL, 60:1490-3, 1988. Exp - Keys : oU - Species : Ar.

[89] U. Becker and D.A. Shirley. Threshold behaviour and resonances in the photoionisation of atoms and molecules. PS, T31:56-66, 1990. Exp. review - Keys : OU AI - Species : Li, Be, Ne, Ar, Kr. 
[90] U. Becker, D. Szostak, H.G. Kerkhoff, M. Kupsch, B. Langer, R. Wehlitz, A. Yagishita and T. Hayaishi. Subshell photoionization of Xe between 40 and 1000 eV. PRA, 39:3902-11, 1989. Exp - Keys : OU IN - Species : Xe.

[91] U. Becker, D. Szostak, M. Kupsch, H.G. Kerkhoff, B. Langer and R. Wehlitz. Decay of the Xe $4 d \rightarrow$ np excitations : resonant shake-off versus shake-up and spectator transitions. JPB, 22:749-62, 1989. Exp - Keys : IN - Species : Xe.

[92] U. Becker, R. Wehlitz, O. Hemmers, B. Langer and A. Menzel. Observation of participator Auger decay following valence photoionization with excitation. PRL, 63:1054-7, 1989. Exp - Keys : OU IN - Species : Ne, Ar.

[93] W. Becker, L.E. Li and J.K. McIver. Polarization effects in two-colour photodetachment of negative ions. JPB, 23:L753-9, 1990. Theory - Keys : FP - Species : $\mathrm{Cl}^{-}$.

[94] W. Becker, S. Long and J.K. McIver. Higher-harmonic production in a model atom with short-range potential. PRA, 41:4112-15, 1990. Theory - Keys : MP - General.

[95] W. Becker, S. Long and J.K. McIver. Short-range potential model for multiphoton detachment of the $\mathrm{H}^{-}$ion. PRA, 42:4416-19, 1990. Theory - Keys : MP - Species : $\mathrm{H}^{-}$.

[96] W. Becker, R.R. Schlicher, M.O. Scully and K. Wodkiewicz. Role of final state effects in above threshold ionization. JOSAB, 4:743-51, 1987. Th. review - Keys : MP - General.

[97] P. Beiersdorger, M. Bitter, S. Von Goeler and K.W. Hill. Experimental study of the x-ray transitions in the heliumlike isoelectronic sequence. PRA, 40:150, 1989. Exp - Keys : IN - Species : He seq with $16 \leq Z \leq 36$ $\left(\mathrm{S}^{1+}, \mathrm{Ar}^{16+}, \mathrm{K}^{17+}, \mathrm{Sc}^{19+}, \mathrm{Ti}^{20+}, \mathrm{V}^{21+}, \mathrm{Cr}^{22+}, \mathrm{Fe}^{24+}, \mathrm{Kr}^{34+}\right)$.

[98] G.I. Bekov, A.T. Tursunov, G. Khasanov and N.B. Eshkobilov. Laser photoionization spectroscopy of highly excited states of gold atoms. OS, 62:163-6, 1987. Exp-Keys : FP OU - Species : Au.

[99] K.L. Bell, K.A. Berrington, P.G. Burke, A. Hibbert and A.E. Kingston. Photoionisation of the $2 \mathrm{p}^{\mathbf{3} 3 \mathrm{~s}^{3}, 5} \mathrm{~S}^{\circ}$, $2 p^{3} 3 p^{3,5}$ p states of atomic oxygen. JPB, 23:2259S-65, 1990. Theory - Keys : 0U - Species: 0.

[100] K.L. Bell, P.G. Burke, A. Hibbert and A.E. Kingston. Photoionisation of the $2 \mathrm{p}^{4}{ }^{3} \mathrm{P},{ }^{1} \mathrm{D},{ }^{1} \mathrm{~S}$ states of atomic oxygen. JPB, 22:3197-204, 1989. Theory - Keys : OU - Species : O.

[101] A.L. Belov and V.P. Krainov. Classical autoionization of two-electron atoms. SPJETP, 65:257-61, 1987. Theory - Keys : AI - Species : He seq.

[102] E.A.J.M. Bente and W. Hogervorst. The $5 \mathrm{dnf} J=4$ and 5 autoionising Rydberg series in barium : experiment and MQDT analysis. JPB, 22:2679-704, 1989. Exp \& theory - Keys : AI - Species : Ba.

[103] E.A.J.M. Bente and W. Hogervorst. The $5 \mathrm{~d} n \mathrm{~d}$ and $5 \mathrm{dng} J=4,3$ and 2 and $5 \mathrm{dns} J=2$ series in barium : linewidths and linestrengths. ZPD, 14:119-28, 1989. Exp-Keys : AI - Species : Ba.

[104] E.A.J.M. Bente and W. Hogervost. Low autoionization rates in the (5dnl) $J=4,5$ doubly excited series in Ba $\mathrm{I}$. PRA, 36:4018-4, 1987. Exp-Keys : AI - Species : Ba.

[105] E.A.J.M. Bente and Hogervorst W. $6 \mathrm{pnf}[J=2,3,4]$ and $6 \mathrm{p} n \mathrm{~h}[J=4,5,6]$ autoionising series of Ba. JPB, 23:1403-21, 1990. Exp - Keys : AI - Species : Ba.

[106] J. Bentley and D.M. Chipman. Partial widths of resonances by analytic continuation from real eigenvalues. CPL, 167:246-51, 1990. Theory - Keys : AI - General.

[107] J. Bentley and D.M. Chipman. Calculation of partial widths for autoionization of the ${ }^{1} \mathrm{P}$ (3s3p) resonance state of helium. JCP, 93:1785-90, 1990. Theory-Keys : AI - Species : He.

[108] H. Bergeron, A. Valance and T.J. Morgan. Calculation of highly excited autoionizing energy states of atomic sodium. ZPD, 14:293-9, 1989. Theory - Keys : AI - Species : Na.

[109] K.A. Berrington, P.G. Burke, K. Butler, M.J. Seaton, P.J. Storey, K.T. Taylor, and Y. Yu. Atomic data for opacity calculations : II. Computational methods. JPB, 20:6379-97, 1987. Theory - Keys : OU - General.

[110] I.J. Bersons. Quasiclassical approach to multiphoton ionization of hydrogen atoms. MP, p. 82-7, 1988. Theory - Keys : FP - Species : H.

[111] I.J. Bersons. WKB theory of multiphoton processes in atoms. JOSAB, 7:617-21, 1990. Theory - Keys : MP General. 
[112] C.P. Bhalla and K.R. Karim. Lifetimes of Be-like levels of argon and titanium. NIMPR, B40-41:369-71, 198: Exp-Keys : AI - Species : $\mathrm{Ar}^{14+}, \mathrm{Ti}^{18+}$.

[113] C.P. Bhalla, K.R. Karim and $M$ Wilson. Autoionization rates for selected double-Rydberg states of barium PRA, 41:1715-17, 1990. Theory - Keys : A - Species : Ba.

[114] S. Bivona, R. Burlon, C. Leone and G. Ferrantc. Effect of a multimode laser field on multichannel multiphoto ionization of hydrogen. JMO, 36:205-14, 1988. Theory - Keys : MP - Species : H.

[115] S. Bivona, R. Burlon, C. Leone and G. Ferrante. Asymmetric angular distributions in two-frequency multiphoto ionization of hydrogen atoms. NCD, 11D:1751-61, 1989. Theory-Keys : MP - Species : H.

[116] J.M. Bizau, D. Cubaynes, P. Gérard, F.J. Wuilleumier and J.L. Picqué. Photoionization cross section mea surement in $5 \mathrm{p}$ and $5 \mathrm{~s}$ subshells of excited barium atoms between $40 \mathrm{eV}$ and $140 \mathrm{eV}$ photon energy. JPCo 48-C9:491-6, 1987. Exp - Keys : IN - Species : Ba.

[117] J.M. Bizau, D. Cubaynes, P. Gérard and F.J. Wuilleumier. Photoionization of atomic barium : Ba atoms in th ground state. PRA, 40:3002-25, 1989. Exp-Keys : OU - Species : Ba.

[118] P.R. Blazewicz, X. Tang, R.N. Compton and J.A.D. Stockdale. Photoelectron angular distributions from reso nantly enhanced multiphoton ionization of xenon via the $6 s[3 / 2]_{1}^{\circ}$ and $6 s[1 / 2]_{1}^{\circ}$ states : experiment and theory JOSAB, 4:770-4, 1987. Exp \& theory - Keys : MP - Species : Xe.

[119] C. Blondel, R.J. Champeau, M. Crance, A. Crubellier, C. Delsart and D. Marinescu. Measurement of the threephoton detachment cross sections of the negative ions of iodine, bromine and fluorine at the wavelength $1.064:$ $\mu \mathrm{m}$. JPB, 22:1335-51, 1989. Exp-Keys : FP-Species : $\mathrm{F}^{-}, \mathrm{Br}^{-}, \mathrm{I}^{-}$.

[120] C. Blondel, M. Crance, C. Delsart, A. Giraud and R. Trainham. Angular distribution in multiphoton detachmen! of $\mathrm{Br}^{-}$. JPB, 23:L685-90, 1990. Exp \& theory - Keys : MP - Species : $\mathrm{Br}^{-}$.

[121] C. Blondel and R. Traiham. Measurement of the four-photon detachement cross-section of $\mathrm{Cl}^{-}$at $1.064 \mu \mathrm{m}$ JOSAB, 6:1774-6, 1989. Exp-Keys:FP-Species : $\mathrm{Cl}^{-}$.

[122] L.A. Bloomfield. Threshold shifts in strong radiation fields; the connection between DC and AC effects. PRL 63:1578-81, 1989. Theory - Keys : SF MP - General.

[123] L.A. Bloomfield. Photodetachment-threshold shifts in two-frequency radiation fields. JOSAB, 7:472-80, 1990 Theory - Keys : MP - General.

[124] $R$. Blumel and U. Smilansky. Ionization of hydrogen Rydberg atoms in strong monochromatic and bichromatic microwave field. JOSAB, 7:664-79, 1990. Theory - Keys : MP - Species : H.

[125] E.G. Bogomol'nyi. Oscillation of the photoabsorption near the ionization threshold for hydrogen-like atoms in electric and magnetic fields. SPJETPL, 47:526-7, 1988. Theory-Keys : SF - Species : H seq.

[126| E.G. Bogomol'nyi. Photoabsorption by atoms in external fields near the ionization threshold. SPJETP, 69:27583, 1989. Theory - Keys : SF - Species : H.

[127] J.J. Bolick and M.S. Banna. Experimental xenon 4d photoionisation cross-sections in the 75-140 eV photor energy range. PRA, 40:2756-7, 1989. Exp-Keys : IN - Species : Xe.

[128] I.I. Bondar, M.I. Dudich, V.V. Saran and L.L. Shimon. Nonlinear ionization of ytterbium atoms. OS, 64:284-6 1988. Exp-Keys : MP MI - Species : Yb.

[129] N. Böwering, M. Salzmann, R. Muller, H.W. Klausing and U. Heinzmann. Photoelectron spin polarizatior approaching unity : photoionisation of Tl. PS, 41:429-32, 1990. Exp-Keys : OU -Species : Tl.

[130] K. Boyer, H. Jara, T.S. Luk, I.A. McIntyre, A. McPherson, R. Rosman, J.C. Solem, C.K. Rhodes and A. Szoke Discussion of the role of many-electron motions in multiphoton ionization and excitation. MP, p. 58-69, 1988 Th. review - Keys : MP MI - Species : Ar, Kr, Xe.

[131] M. Brewczyk and M. Gajda. Mechanism of the multiple ionisation of atoms by strong laser pulses. JPB 21:L383-9, 1988. Theory - Keys : MP MI - General.

[132] L.R. Brewer, F. Buchinger, M. Ligare and D.E. Kelleher. Resonance enhanced multiphoton ionization of atomic hydrogen. PRA, 39:3912-23, 1989. Exp-Keys : MP - Species : H. 
[133] R. Bruzzese, A. Sasso and S. Solimeno. Multiphoton excitation and ionization of atoms and molecules. RNC, 12:1-105, 1989. Review - Keys : MP - General.

[134] P.H. Bucksbaum, M. Bashkansky and D.W. Schumacher. Above-threshold ionization in helium. PRA, 37:361518, 1988. Exp - Keys : MP - Species : He.

[135] P.H. Bucksbaum, R.R. Freeman, M. Bashkansky and T.J. Mcllrath. Role of the ponderomotive potential in above-threshold ionization. JOSAB, 4:760-4, 1987. Review - Keys : MP - Species : Kr, Xe.

[136] P.H. Bucksbaum. Recent progress in above-threshold ionization. AIPCP, 205:499-504, 1990. Theory - Keys : MP - Specjes : Kr, Xe.

[137] P.H. Bucksbaum, L.D. van Woerkom, R.R. Freeman and D.W. Schumacher. Non resonant above-threshold ionization by circularly polarized subpicosecond pulses. PRA, 41:4119-22, 1990. Exp - Keys : MP - Species : Xe.

[138] R. Buffa and S. Cavalieri. Laser-induced autoionization with pulsed excitation. PRA, 42:5481-5, 1990. Theory - Keys : AI MP - General.

[139] R. Buffa and J.K. Spong. An approach to the study of strong-field laser-induced autoionization. NCD, 12D:37789, 1990. Theory - Keys : AI MP - General.

[140] V.V. Bulgakov, V.T. Gavrilyuk, A.P. Lashko, N.V. Stril'chuk, A.I. Feoktistov, Y.E. Frantsev and V.B. Kharlanov. The KLL group Auger electron spectrum of ${ }^{169} \mathrm{Tm}$. BASUPS, 49:23-5, 1986. Exp - Keys : IN - Species : Tm.

[141] P.G. Burke and K.A. Berrington. Resonances in $\mathrm{H}^{-}$converging to the $n$th threshold. JPB, 22:2759-68, 1989 . Theory - Keys : AI - Species : $\mathrm{H}^{-}$.

[142] S.M. Burkov, N.A. Letyaev, S.I. Strakhova and T.M. Zajac. Photon and electron ionisation of helium to the $n=2$ state of $\mathrm{He}^{+}$. JPB, 21:1195-208, 1988. Theory - Keys : OU - Species : He.

[143] S.M. Burkov, N.A. Letyaev and S.I. Strakhova. Photodetachement of the $\mathrm{H}^{-}$ion. PLA, 150:31-5, 1990. Theory - Keys : OU - Species : $\mathrm{H}^{-}$.

[144] S.M. Burkov, N.A. Letyaev and S.I. Strakhova. Resonant photoionization of helium-like ions in the region between the second and third thresholds. Helium-like lithium. MUPB, 44:5-9, 1989. Theory - Keys : 0U Species : $\mathrm{Li}^{+}$.

[145] R. Burlon, S. Basile, F. Trombetta and G. Ferrante. Analytical evaluation of integrals occuring in bound-free transitions. PRA, 37:390-9, 1988. Theory - Keys : MP - Species : H.

[146] K. Burnett. Two-electron bound-free transitions in an intense laser field. JPB, 21:3083-92, 1988. Theory Keys : MP MI - Species : He seq.

[147] K. Burnett. Two-electron excitation and ionization in an intense laser field. JMO, 36:925-40, 1989. Theory Keys : MP MI AI - General.

[148] K. Butler and C.J. Zeippen. Photoionisation of the ${ }^{3} \mathrm{P}^{\mathrm{e}},{ }^{1} \mathrm{D}^{\mathrm{e}}$ and ${ }^{1} \mathrm{~S}^{\mathrm{e}}$ states of neutral oxygen. AA, 234:569-75, 1990. Theory - Keys : OU - Species : O.

[149] V. Buzek. $N$-level atom interacting with single-mode radiation field : an exactly solvable model with multiphoton transitions and intensity-dependent coupling. JMO, 37:1033-53, 1990. Theory - Keys : MP - General.

[150] M. Bylicki. Feshbach-projection calculations of inelastic resonances in $\mathrm{He}^{-}$. PRA, 41:2386-90, 1990. Theory Keys : AI - Species : $\mathrm{He}^{-}$.

[151] C.D. Caldwell, M.O. Krause and J. Jimenez-Mier. Photoionization of gallium at $3 d-4 p$ and 4s-np ( $n=5,6)$ resonances. PRA, 37:2408-14, 1988. Exp-Keys : OU AI - Species : Ga.

[152] C.D. Caldwell and M.O. Krause. Photoionisation of neon between the 2p fine structure limits. JPB, 23:2233-9, 1990. Exp - Keys : OU - Species : Ne.

[153] P. Camus, M. Kompitsas, S. Cohen, C. Nicolaides, M. Aymar, M. Crance and P. Pillet. Multiphoton single and double ionisation of strontium in the range 532-541 nm. JPB, 22:445-58, 1989. Exp \& theory - Keys : MP MI Species : Sr. 
[154] P. Camus, P. Pillet and Boulmer J. Spectroscopy of doubly excited $9 \mathrm{~d} n^{\prime} \mathrm{d}$ states of neutral barium. AtP, 11:213-14, 1986. Exp-Keys : AI - Species : Ba.

[155] A.M. Cantu and W.H. Parkinson. Absorption spectrum of atomic chlorine from $950 \AA$ to $600 \AA$. PS, 37:336-41, 1988. Exp-Keys : OU - Species : Cl.

[156] S. Canuto, J. Geertsen, F. Muller-Plathe and G.F. Srusena. Coupled cluster polarisation propagator study of the photodetachment cross section of $\mathrm{Li}^{-}$. JPB, 21:3:91-7, 1988. Theory - Keys: OU - Species : $\mathrm{Li}^{-}$.

[157] T.A. Carlson, D.R. Millins, C.E. Beall, B.W. Yates, J.W. Taylor, D.W. Lindle, B.P. Pullen and F.A. Grimm. Unusual degree of angular anisotropy in the resonant Auger spectrum of Kr. PRL, 60:1382-5, 1988. Exp Keys : IN - Species : Kr.

[158] T.A. Carlson, D.R. Millins, C.E. Beall, B.W. Yates, J.W. Taylor, D.W. Lindel, and F.A. Grimm. Angular distribution of ejected electrons in resonant Auger processes of Ar, Kr and Xe. PRA, 39:1170-85, 1989. ExpKeys : IN - Species : Ar, Kr, Xe.

[159] H.J. Carmichael, S. Singh, R. Vyas and P.R. Rice. Photoelectron waiting times and atomic state reduction in resonance fluorescence. PRA, 39:1200-18, 1989. Theory - Keys : OU - General.

[160] V. Carravetta. Theoretical analysis of lineshape in core-electron shake-up spectra: Ne 2s-3s satellite. JPB, 21:1777-90, 1988. Theory - Keys : IN - Species : Ne.

[161] V. Carravetta, H. Agren, H.J.A. Jensen, P. Jorgensen and J. Olsen. Accurate photodetachment cross sections for $\mathrm{Li}^{-}$. JPB, 22:2133-40, 1989. Theory - Keys : OU - Species : $\mathrm{Li}^{-}$.

[162 B. Carre, P. d'Oliveira, M. Ferray, P. Fournier, F. Gounand, D. Cubaynes, J.M. Bizau and F.J. Wuilleumier. Photoionization of highly excited atomic sodium involving core-excited resonances. ZPD, 15:117-32, 1990. Exp - Keys : OU AI - Species: Na.

[163] B. Carre, P. d'Oliveira, P.R. Fournier, F. Gounand and M. Aymar. $6 \mathrm{p}_{1 / 2,3 / 2} n \mathrm{p} ; J=0,1,2$ autoionizing series in atomic barium. PRA, 42:6545-59, 1990. Exp \& theory-Keys : A - Species : Ba.

[164] N. Chandra. Angular momentum transfer formalism for spin polarized atomic photoelectrons. PRA, 42:4050-7, 1990. Theory - Keys : OU - Species : Yb.

[165] T.N. Chang. Photoionization of quasi-two-electron atoms dominated by the doubly excited autoionization states. PRA, 37:4090-6, 1988. Theory - Keys : OU - Species : Mg.

[166] T.N. Chang and X. Tang. Photoionization of magnesium from the excited 3snd ${ }^{1} \mathrm{D}$ states to the doubly excited 3 pnl ${ }^{1} \mathrm{~F}$ autoionization states. PRA, 38:1258-62, 1988. Theory-Keys : OU AI - Species : Mg.

[167] M. Chantepie, B. Cheron, J.L. Cojan, J. Landais and B. Laniepce. The Zn I 4p ${ }^{2}$ autoionising level widths. JPB, 21:1379-84, 1988. Exp - Keys : AI - Species : Zn.

[168] D. Charalambidis, R. Brenn and K.J. Koulen. Transition rates of $1 \mathrm{~s} 2 \mathrm{~s} 2 \mathrm{p}{ }^{4} \mathrm{P}_{J}^{\circ}$ states of Li-like ions $(Z=8,7,6)$. PRA, 40:2359-64, 1989. Exp-Keys : IN - Species : $\mathrm{C}^{3+}, \mathrm{N}^{4+}, \mathrm{O}^{5+}$.

[169] D. Charalambidis, B.H. Feng and C. Fotakis. Angular distributions of photoelectrons in resonantly enhanced multiphoton ionization via the $7 \mathrm{~s}[1 \mathrm{1} / 2]_{1}^{\circ}$ and $8 s[1 \quad 1 / 2]_{1}^{\circ}$ states of Xe. ZPD, 14:223-7, 1989. Exp - Keys : MP Species : Xe.

[170] D. Charalambidis, K.J. Koulen and R. Brenn. Radiative and autoionization rates of the metastable $2^{\mathbf{4}} \mathrm{P}_{j}^{\circ}$ states in lithiumlike neon. ZPD, 14:17-23, 1989. Exp-Keys : AI - Species : $\mathrm{Ne}^{7+}$.

[171] B. Chen. A new derivation of Keldysh's formula. ChPL, 7:219-21, 1990. Theory - Keys : MP - General.

[172] B. Chen, F.H.M. Faisal, S. Jetzke, H.O. Lutz and P. Scanzano. Above-threshold electron ejection spectra. PRA, 36:4091-4, 1987. Theory - Keys : MP - General.

[173] B.Z, Chen. Angular distribution of above-threshold ionization of $\mathrm{H}$ atom. APS, 39:40-5, 1990. Theory - Keys : MP - Species : H.

[174] C.E. Chen and D.S. Elliott. Measurements of optical phase variation using interfering multiphoton processes. PRL, 65:1737-40, 1990. Exp - Keys : MP - Species : Ar. 
[175] M.H. Chen. $L$-subshell state-to-state photoionization cross sections for the carbon isoelectronic sequence. PRA, 35:4586-91, 1987. Theory - Keys : oU - Species : C seq with $6 \leq Z \leq 54$ (Xe ${ }^{48+}$ with more details).

[176] M.H. Chen. Relativity : X-ray and Auger transitions of highly charged ions. NIMPR, B43:366-71, 1989. Theory - Keys : IN -- General.

[177] M.H. Chen. Effect of relativity and configuration interaction on $L$-shell Auger and radiative decay of the doubly excited $3 l 3 l^{\prime}$ states of sodiumlike ions. PRA, 40:2365-72, 1989. Theory - Keys : IN - Species : Na seq with $18 \leq Z \leq 26\left(\mathrm{Ar}^{7+}, \mathrm{Ti}^{11+}, \mathrm{Fe}^{15+}\right)$.

[178] M.H. Chen. Effective $L$-shell fluorescence yields for sodiumlike and neonlike autoionizing states. PRA, 40:275861, 1989. Theory - Keys : AI - Species : Ne seq, Na seq.

[179] M.H. Chen and B. Crasemann. Auger and radiative decay rates of is vacancy states in the boron isoelectronic sequence : effects of relativity and configuration interaction. PRA, 35:4577-85, 1987. Theory - Keys : IN Species : B seq with $6 \leq Z \leq 54\left(\mathrm{C}^{+}, \mathrm{N}^{2+}, \mathrm{O}^{3+}, \mathrm{F}^{4+}, \mathrm{Ne}^{5+}, \mathrm{Mg}^{7+}, \mathrm{Si}^{9+}, \mathrm{S}^{1+}, \mathrm{Ar}^{13+}, \mathrm{Ca}^{15+}, \mathrm{Ti}^{17+}, \mathrm{Fe}^{21+}\right.$, $\left.\mathrm{Zn}^{25+}, \mathrm{Kr}^{31+}, \mathrm{Mo}^{37+}, \mathrm{Ag}^{42+}, \mathrm{Xe}^{49+}\right)$.

[180] M.H. Chen and B. Crasemann. $K$-shell Auger and radiative transitions in the boron isoelectronic sequence. ADNDT, 38:381, 1988. Theory - Keys : IN - Species : B seq with $6 \leq Z \leq 26\left(\mathrm{C}^{+}, \mathrm{N}^{2+}, \mathrm{O}^{3+}, \mathrm{F}^{4+}, \mathrm{Ne}^{5+}\right.$, $\left.\mathrm{Na}^{6+}, \mathrm{Mg}^{7+}, \mathrm{Al}^{8+}, \mathrm{Si}^{9+}, \mathrm{P}^{10+}, \mathrm{S}^{1+}, \mathrm{Cl}^{12+}, \mathrm{Ar}^{13+}, \mathrm{K}^{14+}, \mathrm{Ca}^{15+}, \mathrm{Sc}^{16+}, \mathrm{Ti}^{17+}, \mathrm{V}^{18+}, \mathrm{Cr}^{19+}, \mathrm{Mn}^{20+}, \mathrm{Fe}^{21+}\right)$.

[181] M.H. Chen, R.J. Fortner, C.J. Hailey and R.E. Stewart. Observation of two-electron-jump, core changing Xray transitions in fluorinelike and oxygenlike lanthanum. PRL, 61:1186-9, 1988. Exp \& theory - Keys : IN Species : $\mathrm{La}^{48+}, \mathrm{La}^{49+}$.

[182] M.H. Chen, W. Weber and W. Mehlhorn. Very strong correlation effects in Auger spectra through the collapse of $d$ orbitals : the 2p-3p4s Auger spectrum of free Ca atoms. JESRP, 49:77-85, 1989. Theory - Keys : IN Species: Ca.

[183] Z. Chen and C.D. Lin. Systematics of autoionization widths of doubly excited states of atoms. PRA, 40:6712-15, 1989. Theory - Keys : AI - Species : He seq $\left(\mathrm{H}^{-}, \mathrm{He}, \mathrm{C}^{4+}, \mathrm{N}^{5+}, \mathrm{O}^{6+}\right)$.

[181] P. Cheng and H.P. Kelly. Photoionization cross sections of the Ar atom for production of singly and doubly charged ions near the 2p threshold. PRA, 39:6232-40, 1989. Theory-Keys : IN MI - Species : Ar.

[185] N.A. Cherepkov and V.V. Kuznetsov. Optical activity of polarised atoms. JPB, 22:L405-9, 1989. Theory Keys : ou - General.

[186] B. Cheron, J.L. Cojan, J. Landais and M. Aymar. Autoionisation widths of the $\mathrm{Hg}\left(6 \mathrm{p}^{2}\right){ }^{3} \mathrm{P}_{0,1}$ levels. JPB, 22:2129-32, 1989. Exp \& theory - Keys : AI - Species: $\mathrm{Hg}$.

[187] S.I. Chu. Multiphoton processes in intense laser fields. ChJP, 24:1-18, 1986. Review - Keys : MP - General.

[188] S.I. Chu and R.Y. Yin. Classical and quantal nonperturbative treatments of multiphoton and above-threshold ionization. JOSAB, 4:720-5, 1987. Th. review - Keys : MP - General.

[189] K.T. Chung. Saddle-point variation method for autoionizing states of atomic systems. ChJP, 25:215-21, 1987. Th. review - Keys : AI - General.

[190] K.T. Chung. On the 1s2s2p2p P-states of B II. ChJP, 27:507-19, 1989. Theory - Keys : AI - Species : B .

[191] K.T. Chung. Identification of high resolution Auger spectra of boron and beryllium. JPB, 23:2929-43, 1990. Theory - Keys : IN - Species : Be, B.

[192] K.T. Chung. Identification of 1 s core-excited four-electron resonances in high resolution spectra of carbon. PS, 42:530-6, 1990. Theory - Keys : A I - Species : $C^{2+}$.

[193] F. Ciralli, S. Basile, G. Ferrante and F. Trombetta. Role of the initial-state configuration in multiphoton ionization of helium. JPB, 21:L651-7, 1988. Theory - Keys : MP - Species : He.

[194] S. Cohen, H.C. Bryant, C.J. Harvey, J.E. Stewart, K.B. Butterfield, D.A. Clark, J.B. Donahue, D.W. MacArthur, G. Comtet and W.W. Smith. Response of the ${ }^{1} \mathrm{P}^{\circ}$ resonance near $n=3$ in the $\mathrm{H}^{-}$continuum to external electric fields. PRA, 36:4728-36, 1987. Exp-Keys : SF - Species : $\mathrm{H}^{-}$. 
[195] R. Colle and S. Simonucci. The continuum orbital problem in the calculation of Auger decay rates applicatio to Ne. NCD, 11D:1587-602, 1989. Theory - Keys : IN - Species : Ne.

[196] L.A. Collins and A.L. Merts. Model calculation for an atom interacting with an intense time dependent electri field. PRA, 37:2415-31, 1988. Theory-Keys : MP - General.

[197] L.A. Collins and A.L. Merts. Atoms in strong, oscillating electric fields : momentum-space solutions of th time-dependent, three-dimensional Schrödinger equation. JOSAB, 7:647-58, 1990. Theory - Keys : MP General.

[198] Combet-Farnoux F. Giant resonances in heavy elements : open-shell effects, multiplet structure and spin-orbi interaction. NATO2, p. 153-70, 1987. Th. review - Keys : OU - Species : Ag, Au.

[199] F. Combet-Farnoux. Photoionisation of multiply-charged ions in the VUV and X-ray energy ranges. JPCc 49-C7:3-15, 1988. Th. review - Keys : OU IN - Species : $\mathrm{Xe}, \mathrm{Cs}^{+}, \mathrm{Ba}^{2+}, \mathrm{La}^{3+}, \mathrm{Ca}$ ions, Fe ions.

[200] F. Combet-Farnoux. Double excitation sattelites of iodine : theoretical study. JPCo, this issue, 1990 . Theor: - Keys : IN - Species : I.

[201] J.P. Connerade. The VUV is more exciting. CAMP, 17:199-217, 1986. Exp. review - Keys : OU - Species : X€ $\mathrm{Gd}, \mathrm{Tl}, \mathrm{Pb}, \mathrm{Th}$.

[202] J.P. Connerade. Controlled collapse and the profiles of giant resonances. NATO2, p. 3-23, 1987. Exp. review Keys : OU - General.

[203] J.P. Connerade. The theory of Faraday rotation in an autoionising resonance. JPB, 21:L551-5, 1988. Theory Keys : AI - General.

[204] J.P. Connerade, M.A. Baig and M. Sweeney. Centrifugal barrier effects in the 3p spectrum of calcium. JPB 20:L771-5, 1987. Exp - Keys : IN - Species : Ca.

[205] J.P. Connerade and A.M. Lane. $q$ reversals in resonant multiphoton ionisation spectroscopy. JPB, 20:L363-7 1987. Exp - Keys : MP - General.

[206] J.P. Connerade and A.M. Lane. Interacting resonances in atomic spectroscopy. RPP, 51:1439-78, 1988. Reviev - Keys : ou - General.

[207] W.E. Cooke. Closed-form expression for two-photon core excitation of Rydberg states. PRA, 33:3529-30, 1986 Theory - Keys : FP - General.

[208] J.W. Cooper. Near-threshold $K$-shell absorption cross section of argon : relaxation and correlation effects PRA, 38:3417-24, 1988. Theory - Keys : IN - Species : Ar.

[209] J.W. Cooper. Angular distributions of electrons in resonant Auger spectra. PRA, 39:3714-16, 1989. Theory Keys : IN - Species : Ar.

[210] J.W. Cooper. Multipole corrections to the angular distribution of photoelectrons at low energies. PRA, 42:6942 45, 1990. Theory - Keys : ou - General.

[211] J.W. Cooper, C.W. Clark, C.L. Cromer, T.B. Lucatorto, B.F. Sonntag, E.T. Kennedy and J.T. Costello. Marke differences in the $3 p$ photoabsorption between the $\mathrm{Cr}$ and $\mathrm{Mn}^{+}$isoelectronic pair : reasons for the uniqu structure observed in Cr. PRA, 39:6074-7, 1989. Exp \& theory - Keys : IN - Species : Cr, $\mathrm{Mn}^{+}, \mathrm{Mn}$.

[212] D. Cordes and P.L. Altick. Application of the multichannel configuration-interaction theory to the characteristic of the $(3,3 b)^{1}$ S state of helium. PRA, 41:5213-16, 1990. Theory - Keys : A I - Species : He.

[213] P.B. Corkum, N.H. Burnett and F. Brunel. Above-threshold ionization in the long-wavelength limit. PRI 62:1259-62, 1989. Theory-Keys : MP - Species : H, Xe.

[214] R.D. Cowan and M. Wilson. Assignment of resonances in the $K^{+}$photoionisation cross section. JPB, 21:L201-4 1988. Theory - Keys : AI - Species : $\mathrm{K}^{+}$.

[215] R.D. Cowan and M. Wilson. Identification of new autoionisation structures in $\mathrm{Zn}^{+}$. JPB, 21:L275-9, 198\& Theory - Keys : $\mathrm{AI}$ - Species : $\mathrm{Zn}^{+}$.

[216] M. Crance. Multiphoton ionisation towards multiple continua : the role of space charge. JPB, 19:L267-71, 1986 Theory - Keys : MP MI - General. 
[217] M. Crance. The influence of space charge on the angular distribution of electrons ejected after multiphoton ionisation. JPB, 19:L671-6, 1986. Theory - Keys : MP - General.

[218] M. Crance. Multiphoton detachment from negative ions of halogens. JPB, 20:6553-62, 1987. Theory - Keys : MP - Species : $\mathrm{F}^{-}, \mathrm{Cl}^{-}, \mathrm{Br}^{-}, \mathrm{I}^{-}$.

[219] M. Crance. Multiphoton stripping of atoms. PRp, 144:117, 1987. Review - Keys : MP - General.

[220] M. Crance. Electron energy spectrum after multiphoton ionisation. JPB, 21:1987-95, 1988. Theory - Keys : MP - General.

[221] M. Crance. Multiphoton ionisation of hydrogen. A non-perturbative calculation of strong-field effects. JPB, 21:2697-708, 1988. Theory - Keys : MP - Species : H.

[222] M. Crance. Multiphoton detachment from negative ions of halogens, angular distributions and excess photon absorption. JPB, 21:3559-69, 1988. Theory - Keys : MP -Species : $\mathrm{F}^{-}, \mathrm{Cl}^{-}, \mathrm{Br}^{-}, \mathrm{I}^{-}$.

[223] M. Crance. Above-threshold two-photon detachment from $\mathrm{H}^{-}$: a way to measure ${ }^{1} \mathrm{~S}$ and ${ }^{1} \mathrm{D}$ resonances. JPB, 21:L557-60, 1988. Theory - Keys : MP - Species : $\mathbf{H}^{-}$.

[224] M. Crance. Role of the space charge in above-threshold ionization. SJQE, 18:718-30, 1988. Theory - Keys : MP - General.

225] M. Crance. Multiphoton detachment from negative atomic ions. CAMP, 24:93-108, 1990. Review - Keys : FP - Species : $\mathbf{F}^{-}$.

[226] M. Crance. Multiphoton detachment from $\mathrm{H}^{-}$. JPB, 23:L285-90, 1990. Theory - Keys : MP - Species : $\mathrm{H}^{-}$.

[227] M. Crance. Near-threshold two-color photodetachment of $\mathrm{H}^{-}$. JPB, 23:L677-84, 1990. Theory - Keys : FP Species : $\mathbf{H}^{-}$.

[228] B. Crasemann. Atomic inner-shell threshold excitation phenomena. JPCo, 48-C9:389-400, 1987. Exp. reviewKeys : IN - General.

[229] B. Crasemann. Photoionization phenomena near threshold. CAMP, 22:163-72, 1989. Th. review - Keys : OU IN - General.

[230] O.H. Crawford. Oscillations in photodetachment cross sections for negative ions in magnetic fields. PRA, 37:2432-40, 1988. Theory - Keys : SF - General.

[231] J.D. Cresser. Theory of electron detection and photon-photoelectron correlations in two-photon ionization. JOSAB, 6:1492-503, 1989. Theory - Keys : FP - General.

[232] D.S. Crothers and D.J. Lennon. Semiclassical $(\gamma, 2 \mathrm{e})$ absolute cross sections for $\mathrm{He}^{-}\left({ }^{4} \mathrm{P}_{5 / 2}^{\circ}\right)$ at threshold. JPB, 21:409-12, 1988. Theory - Keys : MI - Species : $\mathrm{He}^{-}$.

[233] D. Cubaynes, J.M. Bizau, F.J. Wuilleumier, D.L. Ederer, J.L. Picqué, B. Carre, M. Ferray and F. Gounand. The main phases of development in photoemission studies on laser excited atoms using synchrotron radiation. JPCo, 48-C9:453-72, 1987. Exp. review - Keys : FP OU - Species : Na, Ba.

[234] D. Cubaynes, J.M. Bizau, T.J. Morgan, F.J. Wuilleumier, M. Ferray, F. Gounand, P. d'Oliveira and P.R. Fournier. Inner shell excitation in highly excited sodium atoms from the combined use of synchrotron radiation and two laser beams. JPCo, 48-C9:513-21, 1987. Exp-Keys : FP IN - Species : Na.

[235] D. Cubaynes, J.M. Bizau, F.J. Wuilleumier, B. Carre and F. Gounand. Inner-shell photoionization in an excited atom : many-electron effects and partial cross sections in $2 \mathrm{p}^{6} 3 \mathrm{p}^{2} \mathrm{P}_{3 / 2}$ sodium atoms. PRL, 63:2360-3, 1989 . Exp - Keys : IN - Species : Na.

[236] D. Cubaynes, J.M. Bizau, B. Carre and F.J. Wuilleumier. Photoionization of laser excited atoms by synchrotron radiation. AIPCP, 205:224-232, 1990. Exp-Keys : OU - Species : Li, Na.

[237] L.E. Cuellar, R.N. Compton, H.S. Carman Jr and C.S. Feigerle. Photoelectron angular distributions for ns $(n=8-12)$ subshells of cesium : relativistic effects. PRL, 65:163-6, 1990. Exp - Keys : OU - Species : Cs.

[238] C.J. Dai, S.M. Jaffe and T.F. Gallagher. Ba $5 \mathrm{~d}_{5 / 2} n \mathrm{~d}_{J}, J=4$ states and their interaction with the $5 \mathrm{~d}_{3 / 2} \epsilon \mathrm{d}_{5 / 2}$, $J=4$ continuum. JOSAB, 6:1486-91, 1989. Exp-Keys : AI - Species : Ba. 
[239] C.J. Dai, G.W. Schinn and T.F. Gallagher. $\mathrm{Mg} 3$ pnd $(J=3)$ autoionization spectra using isolated-core excitation. PRA, 42:223-35, 1990. Exp-Keys : AI - Species : Mg.

[240] K. Dalwoo. Laser intensity and polarisation effects on the multiphoton ionization of magnesium atoms. JKPS, 23:191-8, 1990. Exp - Keys : MP - Species : Mg.

[241] S. Danzan and S.I. Strakhova. Spectroscopy of autoionizing states of the strontium atom in photoprocesses. JPB, 23:3545-56, 1990. Theory - Keys : AI - Species : Sr.

[242] B.F. Davis and K.T. Chung. Spin-induced autoionization and radiative transition rates for the (1s2p2p) ${ }^{4} P_{J}$ states in lithiumlike ions. PRA, 37:111-118, 1988. Theory-Keys : AI - Species : Li seq with $3 \leq Z \leq 10$ (Li, $\mathrm{Be}^{+}, \mathrm{B}^{2+}, \mathrm{C}^{3+}, \mathrm{N}^{4+}, \mathrm{O}^{5+}, \mathrm{F}^{6+}, \mathrm{Ne}^{7+}$ ).

[243] B.F. Davis and K.T. Chung. Spin-induced autoionization and radiative transition rates for the (1s2s2p) ${ }^{4} P_{J}^{\circ}$ states in lithiumlike ions. PRA, 39:3942-55, 1989. Theory - Keys : AI - Species : Li seq with $3 \leq Z \leq 18$ (Li, $\left.\mathrm{Be}^{+}, \mathrm{B}^{2+}, \mathrm{C}^{3+}, \mathrm{N}^{4+}, \mathrm{O}^{5+}, \mathrm{F}^{6+}, \mathrm{Ne}^{7+}, \mathrm{Na}^{8+}, \mathrm{Mg}^{9+}, \mathrm{Al}^{10+}, \mathrm{Si}^{11+}, \mathrm{P}^{12+}, \mathrm{S}^{13+}, \mathrm{Cl}^{14+}, \mathrm{Ar}^{15+}\right)$.

[244] B.F. Davis and K.T. Chung. Energy and autoionization width of the $1 \mathrm{~s} 3 \mathrm{~s} 3 \mathrm{p}{ }^{4} \mathrm{P}^{\circ}$ and $1 \mathrm{~s} 3 \mathrm{p} 3 \mathrm{p}{ }^{4} \mathrm{P}^{\mathrm{e}}$ states in lithiumlike ions. PRA, 41:5844-55, 1990. Theory - Keys : AI - Species : Li seq with $2 \leq Z \leq 6\left(\mathrm{He}^{-}, \mathrm{Li}, \mathrm{Be}^{+}\right.$, $\left.\mathrm{B}^{2+}, \mathrm{C}^{3+}\right)$.

[245] B.F. Davis and K.T. Chung. Radiative decay of triply excited $2 \mathrm{p}^{2} \mathrm{np}{ }^{4,2} \mathrm{~S}^{\circ}$ states to the $1 \mathrm{~s} 2 \mathrm{pmp}{ }^{4,2} \mathrm{P}$ states in lithiumlike ions. PRA, 42:5121-31, 1990. Theory-Keys : AI - Species : Li seq with $3 \leq Z \leq 10\left(\mathrm{Li}, \mathrm{Be}^{+}, \mathrm{B}^{2+}\right.$, $\left.\mathrm{C}^{3+}, \mathrm{N}^{4+}, \mathrm{O}^{5+}, \mathrm{F}^{6+}, \mathrm{Ne}^{7+}\right)$.

[246] F.X. de Araujo and D. Petrini. 2s photoionisation of atomic Na : 3s3p shake-up process. JPB, 21:L117-9, 1988. Theory - Keys : IN - Species : Na.

[247] F.X. de Araujo and D. Petrini. 2s photoionisation of atomic sodium : 3s $\rightarrow$ 4s, 4p, 4d shake-up processes. JPB, 22:L33-4, 1989. Theory-Keys : IN - Species : Na.

[248] R.J. de Graff, J.M. Bente, W. Hogervorst and A. Wannstrom. Observation of hyperfine structure and isotope shift in autoionizing states. PRA, 37:4532-5, 1988. Exp - Keys : AI - Species : Ba.

[249] R.J. de Graff, W. Ubachs, W. Hogervorst and M. Abutaleb. Narrow $6 \mathrm{p}_{1 / 2,3 / 2}$ p $J=1$ autoionizing Rydberg series in barium and their interaction with $6 p_{3 / 2}$ nf states. PRA, 42:5473-80, 1990. Exp - Keys : AI - Species : Ba.

[250] P. Decleva, G. de Alti, G. Fronzoni and A. Lisini. Theoretical study of the sattelite structure in the photoelectron spectra of neon and argon. JPB, 23:3777-84, 1990. Theory - Keys : IN - Species : Ne, Ar.

[251] P. Decleva and A. Lisini. Theoretical study of the photoelectron spectrum of cesium. NATO1, p. 657-62, 1986. Theory - Keys : oU - Species : Cs.

[252] J.L. Dehmer, S.T. Pratt and P.M. Dehmer. Three-photon excitation of autoionizing states of atomic krypton between the ${ }^{2} \mathrm{P}_{3 / 2}^{\circ}$ and ${ }^{2} \mathrm{P}_{1 / 2}^{\circ}$ fine-structure thresholds. PRA, 36:4494-7, 1987. Exp - Keys : AI FP - Species $\mathrm{Kr}$.

[253] A. Dellafiore and F. Matera. Dynamic response of the Thomas-Fermi atom. PRA, 41:4958-65, 1990. Theory Keys : OU - General.

[254] N.B. Delone and M.V. Fedorov. Above threshold ionization. PQE, 13:267-98, 1989. Review - Keys : MP General.

[255] N.B. Delone and M.V. Fedorov. New effects in the multiphoton ionization of atoms. SPU, 32:500-20, 1989 Exp. review - Keys : MP - General.

[256] P.C. Deshmukh, G. Nasreen and S.T. Manson. Photoionization of $\mathrm{Al}^{+}$ions : a relativistic random-phaseapproximation study. PRA, 38:504-5, 1988. Theory-Keys : ou - Species : $\mathrm{Al}^{+}$.

[257] R.D. Deslattes, E.G. Kessler, Y.K. Kim and P. Indelicato. Systematics of X-ray transition energies for high-Z atoms. JPCo, 48-C9:591-5, 1987. Review - Keys : IN - Species : All $40 \leq Z \leq 99$.

[258] M. Deutsch. the K $\alpha$ satellite structure of sodium. JPB, 20:L681-4, 1987. Theory - Keys : IN - Species : Na. 
[259] P.L. DeVries. Calculation of harmonic generation during the multiphoton ionization of the hydrogen atom. JOSAB, 7:517-20, 1990. Theory - Keys : MP - Species : H.

[260] D. Ding, M. Jin, H. Liu and K.T. Lu. Angular distribution of photoelectrons in nonresonant multiphoton ionization of atomic lead. ChPL, 6:126-9, 1989. Exp-Keys : MP - Species : Pb.

[261] R. Ding, W.G. Kaenders, J.P. Marengos, N. Schen, J.P. Connerade and M.H.R. Hutchinson. Laser spectroscopy of atomic inner shells. JPB, 22:L251-6, 1989. Exp - Keys : IN - Species : Hg.

[262] S.N. Dixit, Levin D.A. and B.V. McKoy. Resonant enhanced multiphoton ionization studies in atomic oxygen. PRA, 37:4220-8, 1988. Theory - Keys : FP - Species : O.

[263] J.P. Doering, M.A. Coplan, J.W. Cooper and J.H. Moore. Electron correlation in an Auger process. PRA, 41:535-8, 1990. Exp - Keys : IN MI - Species : Ar.

[264] V.K. Dolmatov. Marked differences in the $4 s$ photoionization between the $\mathrm{Cr}\left(4 \mathrm{~s}^{7} \mathrm{~S}_{3}\right)$ and $\mathrm{Cr}^{*}\left(4 \mathrm{~s}{ }^{5} \mathrm{~S}_{2}\right)$ atoms. JPB, 23:L625-28, 1990. Theory - Keys : OU - Species : Cs.

[265] V.K. Dolmatov and T.T. Riskiev. Photoionization of the $4 \mathrm{~s}$ level in the chromium atom in the 7-35 eV region of photon energies. OS, 64:705-6, 1988. Theory-Keys : ov - Species : Cr.

[266] Q. Dong, Y. Kuang and R.H. Pratt. Photoionization of atoms and ions. NIMPR, A280:189-94, 1989. Theory - Keys : DU - Species : Ne ions, Ar ions, Fe ions, U ions.

[267] M. Dorr and R.M. Potvliege. Resonances that originate from shadow poles of the scattering matrix in multiphoton processes. PRA, 41:1472-6, 1990. Theory - Keys : MP - General.

[268] M. Dorr, R.M. Potvliege and R. Shakeshaft. Multiphoton processes in an intense laser field. III. Resonance ionization of hydrogen by subpicosecond pulses. PRA, 41:558-61, 1990. Theory - Keys : MP - Species : H.

[269] M. Dorr, R.M. Potvliege, D. Proulx and R. Shakeshaft. Multiphoton detachment of $\mathrm{H}^{-}$and the applicability of the Keldysh approximation. PRA, 42:4138-50, 1990. Theory - Keys : MP - Species : $\mathrm{H}^{-}$.

[270] M. Dorr, R.M. Potvliege and R. Shakeshaft. Atomic hydrogen irradiated by a strong laser field : Sturmian basis calculation of rates for high-order multiphoton ionization, Raman scattering and harmonic generation. JOSAB, 7:433-48, 1990. Theory - Keys : MP - Species : H.

[271] M. Dorr and R. Shakeshaft. Resonance enhancement of above-threshold peaks in multiphoton ionization. PRA, 38:543-6, 1988. Theory - Keys : MP - General.

[272] M. Dorr and R. Shakeshaft. Two-color photodetachment of a model one-dimensional ion. PRA, 40:459-62, 1989. Theory - Keys : FP - Species : $\mathrm{Cl}^{-}$.

[273] S.W. Downey and R.S. Hozack. Saturation of three-photon ionization of atomic hydrogen and deuterium at 243 nm. OL, 14:15-17, 1988. Exp-Keys : MP - Species : H.

[274] R.J. Drachman, A.K. Bhatia and A.A. Shabazz. Two-photon transitions in hydrogen: A test of pseudostate summation. PRA, 42:6333-5, 1990. Theory - Keys : FP - Species : H.

[275] E.G. Drukarev, M.I. Strikman and M.B. Trzhaskovskaya. Ionization of outer electron shells accompanying internal conversion. SJNP, 48:423, 1989. Theory-Keys : IN MI - Species : Tm.

[276] P.D. Drummond. Multiphoton-ionization potentials with dynamical renormalization. PRA, 39:2718, 1989. Theory - Keys : MP - General.

[277] M.L. Du and J.B. Delos. Photodetachment of $\mathrm{H}^{-}$in an electric field. PRA, 38:5609-16, 1988. Theory - Keys : $\mathrm{SF}-$ Species : $\mathrm{H}^{-}$.

(278) M.L. Du. Photodetachment spectra of $\mathrm{H}^{-}$in parallel electric and magnetic fields. PRA, 40:1330-39, 1989. Theory-Keys : SF - Species : $\mathbf{H}^{-}$.

[279] M.L. Du. Oscillations of electron flux in photodetachment of $\mathrm{H}^{-}$in an electric field. PRA, 40:4983-7, 1989. Theory - Keys : SF - Species : $\mathrm{H}^{-}$.

[280] M.L. Du and J.B. Delos. Effects of an electric field on the photodetachment of $\mathrm{H}^{-}$. PLA, 134A:476-9, 1989. Theory - Keys : $\mathrm{SF}-$ Species : $\mathrm{H}^{-}$. 
[281] C. Dzionk, W. Fiedler, C. Kortenkamp, M.V. Lucke and P. Zimmermann. Single and double photoionization of lanthanide elements in the region of the 5p excitation. JPCo, 48-C9:501-4, 1987. Exp - Keys : IN MI Species : Ce, Tb, Yb.

[282] C. Dzionk, W. Fiedler, M. von Lucke and P. Zimmermann. Photoion spectroscopy in the 4d giant resonances of the lanthanides. PRL, 62:878-80, 1989. Exp-Keys : IN - Species : La, Ce, Pr, Nd, Gd, Tb, Dy.

[283] C. Dzionk, W. Fielder, M.V. Lucke and P. Zimmermann. Photoion yield spectra of the lanthanides in the region of the 5p excitation : the elements Gd, Tb, Dy, Ho, Er, Tm and Yb. PRA, 41:3572-4, 1990. Exp - Keys : IN Species: Gd, Tb, Dy, Ho, Er, Tm, Yb.

[284] C. Dzionk, W. Fielder, M. Lucke and P. Zimmerman. Photoion yield spectra of singly and doubly charged lanthanides in the region of the 5p excitation : the elements $\mathrm{La}, \mathrm{Ce}, \mathrm{Pr}, \mathrm{Nd}, \mathrm{Sm}$ and Eu, PRA, 39:1780-2, 1989. Exp-Keys : IN - Species : $\mathrm{La}^{2+}, \mathrm{La}^{+}, \mathrm{Ce}^{2+}, \mathrm{Ce}^{+}, \mathrm{Pr}^{2+}, \mathrm{Pr}^{+}, \mathrm{Nd}^{2+}, \mathrm{Nd}^{+}, \mathrm{Sm}^{2+}, \mathrm{Sm}^{+}, \mathrm{Eu}^{2+}, \mathrm{Eu}^{+}$.

[285] W. Eberhardt, S. Bernstorff, H.W. Jochims, S.B. Whitfield and B. Crasemann. Photoelectron recapture through post-collision interaction. PRA, 38:3808-11, 1988. Exp \& theory - Keys : IN - Species : Ar.

[286] J.H. Eberly. Intensity dependence of strong-field photodetachment rate. JPB, 23:L619-24, 1990. Theory Keys : MP - Species : $\mathrm{H}^{-}$.

[287] J.H. Eberly, J. Javanainen and Q.C. Su. Bound-level 'multiplets' in photoelectron spectra of above-threshold ionization. SJQE, 18:738-40, 1988. Theory - Keys : MP - General.

[288] J.H. Eberly and J. Javanainen. Short-pulse above-threshold ionisation "multiplet" effects. PRL, 60:1346, 1988. Theory - Keys : MP - Species : Xe.

[289] J.H. Eberly, Q. Su, W.G. Greenwood and J. Javanainen. Response of single electron in atoms and negative ions to short pulses of intense radiation. APPA, A78:105-13, 1990. Theory - Keys : MP - Species : $\mathrm{H}^{-}$.

[290] J.H. Eberly, Q. Su and J Javanainan. High harmonic generation at atomic electrons in intense laser fields. BASUPS, 53:74-7, 1989. Theory - Keys : MP - General.

[291] M. Edwards and R. Shakeshaft. Multiphoton ionization by intense fields : corrections to lowest order perturbation theory, with an application to photoionization of H. ZPD, 8:51-6, 1988. Theory - Keys : MP - Species : H.

[292] U. Eichmann, J.L. Dexter, E.Y. Xu and T.F. Gallagher. Microwave ionization and excitation of Ba Rydberg atoms. ZPD, 11:187, 1989. Exp-Keys : MP - Species : Ba.

[293] A.Yu. Elizarov and N.A. Cherepkov. Two-photon polarization spectroscopy of autoionizing states. SPJETP, 69:695-9, 1989. Exp-Keys : AI FP - Species : Ba.

[294] B. Eriksson, S. Svensson, N. Martensson and U. Gelius. The high energy excited shake-up electron spectra of krypton. JPCo, 48-C9:531-4, 1987. Exp-Keys : IN - Species : Kr.

[295] B. Eriksson, S. Svensson, N. Martensson and U. Gelius. The 2p shake-up spectrum of argon : effects of intermediate coupling. JPB, 21:1371-8, 1988. Exp-Keys : IN - Species : Ar.

[296] I.I. Fabrikant. Rescattering of photodetached electrons in a Stark field. PRA, 40:2373-7, 1989. Theory - Keys : $\mathrm{SF}-$ Species : $\mathrm{H}^{-}$.

[297] I.I. Fabrikant. Spatial distribution of electrons photodetached in an electric field. JPB, 23:1139-47, 1990. Theory - Keys : SF - Specjes : $\mathbf{H}^{-}$.

[298] B. Fain and S.H. Lin. General asymptotic behaviors for amplitude and frequency dependence of multiphoton ionization rates. CPL, 165:229-31, 1990. Theory - Keys : MP - General.

[299] F.H.M. Faisal. Theory of multiphoton processes. Plenum Press, 1987. Th. review - Keys : MP - General.

[300] F.H.M. Faisal. Floquet Green's function method for radiative electron scattering and multiphoton ionization in a strong laser field. CPR, 9:55-113, 1989. Th. review - Keys : MP - General.

[301] F.H.M. Faisal, P. Filipowicz and R. Rzazewski. Three-dimensional-model study of above-threshold photodetachment. PRA, 41:6176-82, 1990. Theory - Keys : MP - General. 
[302] F.H.M. Faisal, P. Scanzano and J. Zaremba. Analytical ATI spectrum of an exactly solvable 3D model of laser-atom interaction. JPB, 22:L183, 1989. Theory - Keys : MP - General.

[303] U. Fano. Sharp and diffuse interlopers in excitation spectra. PRA, 37:4037-9, 1988. Theory - Keys : AI General.

[304] J.W. Farley. Photodetachment cross sections of negative ions : the range of validity of the Wigner threshold law. PRA, 40:6286-92, 1989. Theory - Keys : OU - General.

[305] D. Farrelly and T. Uzer. Resonance overlap structure in the microwave ionization of the hydrogen atom. PRA, 38:5902-5, 1988. Theory - Keys : MP - Species : H.

[306] M.V. Fedorov. Strong field coherence effects in photoionization from or via Rydberg levels. APPA, A78:115-22, 1990. Theory - Keys : MP - General.

[307] M.V. Fedorov, M.Y. Ivanov and P.B. Lerner. Interaction of atoms with supershort laser pulses and the generation of the supercontinuum. JPB, 23:2505-20, 1990. Theory - Keys : MP - General.

[308] M.V. Fedorov and M.Yu Ivanov. Coherence and interference in a Rydberg atom in a strong laser field : excitation, ionization and emission of light. JOSAB, 7:569-73, 1990. Theory - Keys : MP - General.

[309] M.V. Fedorov and A.E. Kazakov. Resonances and saturation in multiphoton bound-free transitions. PQE, 13:1-106, 1988. Review - Keys : MP - General.

[310] M.V. Fedorov and A.M. Movsesian. Field-induced effects of narrowing of photoelectron spectra. JPB, 21:L155-8, 1988. Theory - Keys : MP - General.

[311] M.V. Fedorov and A.M. Movsesian. Interference suppression of photoionization of Rydberg atoms in a strong electromagnetic field. JOSAB, 6:928-36, 1989. Theory - Keys : MP - General.

[312] D. Feldman. Multiphoton ionization of atomic hydrogen in intense sub-ps laser pulses. CAMP, 24:311-26, 1990. Exp. review - Keys : MP - Species : H.

[313] D. Feldmann, B. Wolff, M. Wemhoner and K.H. Welge. Above threshold ionization of atomic hydrogen : angular distribution of photoelectrons. MP, p. 35-42, 1988. Exp. review - Keys : MP - Species : H.

[314] Z. Felfi and S.T. Manson. Influence of shape resonances on minima in cross sections for photoionization of excited atoms. PRA, 41:1709-10, 1990. Theory - Keys : OU - Species : At, Rn, Fr.

[315] J.A. Fernley, K.T. Taylor and M.J. Seaton. Atomic data for opacity calculations : VII. Energy levels, $f$ values and photoionisation cross sections for He-like ions. JPB, 20:6457, 1987. Theory - Keys : OU - Species : He seq with $2 \leq Z \leq 10\left(\mathrm{He}, \mathrm{Li}^{+}, \mathrm{Be}^{2+}, \mathrm{Ne}^{8+}\right)$.

[316] M.G.J. Fink and P. Zoller. Quantum defect parametrization of perturbative two-photon ionization cross sections. PRA, 39:2933-47, 1989. Theory - Keys : FP - Species : H.

[317] M. Finkenthal, Litman A., P. Mandelbaum, D. Stutman and J.L. Schwob. High-resolution autoionizing line spectra of $\mathrm{Mg}$ II and Al III in the 160-260 $\AA$ range emitted from a Penning ionization discharge plasma. JOSAB, 5:1640-4, 1988. Exp-Keys : AI - Species : $\mathrm{Mg}^{+}, \mathrm{Al}^{2+}$.

[318] E. Fiordilino, R. Zangara and G. Ferrante. Laser-induced modifications of selection rules in the Auger effect. PRA, 38:4369-72, 1988. Theory - Keys : MP IN - General.

[319] R.R. Freeman, P.H. Bucksbaum and H. Milchberg. Investigations of the role of ponderomotive forces in ATI using subpicosecond laser pulses. MP, p. 2-11, 1988. Exp. review - Keys : MP - Species : Xe.

[320] Z. Fried and L. Kannenberg. Radiative and nonradiative transitions in atomic systems. PLA, 116A:441-3, 1986. Theory - Keys : IN - General.

[321] C. Froese-Fischer. Perturbers in the $\left({ }^{2} \mathrm{D}^{\circ}\right)$ nd ${ }^{3} \mathrm{P}^{\circ}$ Rydberg series of sulphu. JPB, 20:4365-74, 1987. Theory Keys : AI - Species : S.

[322] C. Froese Fischer and M. Idrees. Autoionization rates for core excited ${ }^{5} \mathrm{P}$ states in $\mathrm{Na}^{-}$. PS, 39:70-2, 1989. Theory - Keys : AI - Species : $\mathrm{Na}^{-}$.

[323] C. Froese-Fischer and M Idrees. Spline methods for resonances in photoionisation cross sections. JPB, 23:679-91, 1990. Theory - Keys : OU AI - Species : He. 
[324] H. Fukuda, N. Koyama and M. Matsuzawa. High-lying doubly excited states of $\mathrm{H}^{-}$and He. JPB, 20:2959-74, 1987. Theory - Keys : AI - Species : $\mathrm{H}^{-}$, He.

[325] T.F. Gallagher. Doubly excited states. JOSAB, 4:794-804, 1987. Exp. review - Keys : AI - Species : Ba.

[326] T.F. Gallagher. Above-threshold ionization in low-frequency limit. PRL, 61:2304-7, 1988. Exp - Keys : MP Species : Na.

[327] T.F. Gallagher, C.R. Mahon, P. Pillet, P. Fu and J.B. Newman. Microwave ionization of hydrogenlike Li and $\mathrm{Na}$ atoms. PRA, 39:4545-9, 1989. Exp-Keys : MP - Species : $\mathrm{Li}^{2+}, \mathrm{Na}^{10+}$.

[328] T.F. Gallagher, Y. Zhu and R.R. Johns. Multiphoton double ionisation of barium. PSPIE, 875:48-51, 1988. Exp - Keys : MP MI - Species : Ba.

[329] E.J. Galvez, B.E. Sauer, L. Moorman, P.M. Koch and D. Richards. Microwave ionization of $\mathbf{H}$ atoms : breakdown of classical dynamics for high frequencies. PRL, 61:2011-14, 1988. Exp-Keys : MP - Species : H.

[330] B. Gao, C. Pan, C.R. Liu and A.F. Starace. Variational methods for high-order multiphoton processes in atoms. JOSAB, 7:622--30, 1990. Theory - Keys : MP - Species : He, Ar.

[331] B. Gao and A.F. Starace. Variational calculation of multiphoton ionisation processes for the H atom. PRL, 61:404-7, 1988. Theory - Keys : MP - Species : H.

[332] B. Gao and A.F. Starace. Variational principle for high-order perturbations with application to multiphoton processes for the H atom. PRA, 39:4550-60, 1989. Theory - Keys : MP - Species : H.

[333] W.R.S. Garton and J.P. Connerade. Some complex spectra of simple atoms. JOSAB, 5:2119-24, 1988. Exp. review - Keys : OU - Species : Sc, Mn, Y, Cs , Ba, Gd, Tl, Th.

[334] A.D. Gazazyan and R.G. Unanyan. Influence of a strong external electromagnetic field on autoionizing states of atoms. SPJETP, 66:909-15, 1988. Theory - Keys : AI MP - General.

[335] J. Gea-Banacloche. Two-photon absorption of nonclassical light. PRL, 62:1603, 1989. Theory - Keys : FP General.

[336] S. Geltman. Multiphoton detachment of an electron from $\mathrm{H}^{-}$. PRA, 42:6958-61, 1990. Theory - Keys : MP Species : $\mathrm{H}^{-}$.

[337] G.K. Gerke and B.A. Bushaw. Measurement of weak branching out of the (near-) two-level system Ba 6s6p ${ }^{1} P_{1}$ $\leftrightarrow 6 \mathrm{~s}^{2}{ }^{1} \mathrm{~S}_{\mathrm{O}}$. PRA, 37:1502-6, 1988. Exp-Keys : OU - Species : Ba.

[338] R. Gersbacher and J.T. Broad. Resonances in helium photoionisation. JPB, 23:365-84, 1990. Theory - Keys : AI - Species : He.

[339] F. Giammanco and E. Arimondo. Evidence of charge collective effects in Na laser photoionization. EL, 11:31-6, 1990. Exp - Keys : FP - Species : Na.

[340] G. Gibson, T.S. Luk and C.K. Rhodes. Tunneling ionization in the multiphoton regime. PRA, 41:5049-52, 1990. Exp - Keys : MP - General.

[341] I.B. Goldberg and R.H. Pratt. Possible narrow and deep minima in the photoionization cross sections of excited states. PRA, 36:2108-11, 1987. Theory-Keys : OU - Species : Cs.

[342] P.A. Golovinskii and A.V. Berdyshel. Stripping of inner atomic electrons by an intense laser field. STPL 13:85-6, 1987. Exp - Keys : MP IN - General.

[343] P.A. Golovinsky, I.Y. Kiyan and V.S. Rostovtsev. Influence of dynamical correlation effects on multiphoton processes in negative ions. JPB, 23:2743-59, 1990. Theory - Keys : MP - Species : $\mathrm{F}^{-}, \mathrm{Na}^{-}, \mathrm{Cl}^{-}$.

[344] Y. Gontier, N.K. Rahman and M. Trahin. Resonant multiphoton ionization of atomic hydrogen. PRA, 37:4694701, 1988. Theory - Keys : MP - Species : H.

[345] Y. Gontier and M. Trahin. The role played by the level-shift operator in above-threshold-ionization. JPB 22:2531-9, 1989. Theory - Keys : MP - General.

[346] Y. Gontier and M. Trahin. Angular distributions of photoelectrons from multiresonant ionization of atomik hydrogen at $608 \mathrm{~nm}$. JOSAB, 7:464-6, 1990. Theory - Keys : MP - Species : H. 
[347] Y. Gontier and M. Trahin. Effect of an intense radiation field on the discrete and continuum spectra of atomic hydrogen. PRA, 40:1351-62, 1989. Theory - Keys : MP - Species : H.

[348] S.P. Goreslavskii and O.V. Shcherbachev. Ponderomotive broadening in above threshold ionization spectra. SPJETP, 68:53-58, 1989. Theory - Keys : MP - General.

[349] F. Gounand. Spectroscopy of highly excited two-electron atoms: an overview. APPA, A78:141-58, 1990. Review - Keys : AI - Species : He seq.

[350] D.V. Grabauskas and J.J. Grudzinskas. Energy spectrum of auto-ionization electrons from the decay of Ba ${ }^{+}$ ions. SPC, 28:89-90, 1989. Exp-Keys : AI - Species : $\mathrm{Ba}^{+}$.

[351] H. Gratl, G. Alber and P. Zoller. Near-threshold behaviour of multiphoton ionisation probabilities. JPB, 22:L_547-51, 1989. Theory - Keys : MP - General.

[352] C.H. Greene. Multiple excitations of atomic electrons. JOSAB, 4:775-83, 1987. Review - Keys : AI - Species : $\mathrm{Be}, \mathrm{Mg}, \mathrm{Ar}, \mathrm{Ca}, \mathrm{Ge}, \mathrm{Kr}, \mathrm{Sn}, \mathrm{Xe}, \mathrm{Ba}$.

[353] C.H. Greene. Negative-ion photodetachment in a weak magnetic field. PRA, 36:4236-44, 1987. Theory - Keys : $\mathrm{SF}-$ Species : $\mathrm{H}^{-}$.

[354] C.H. Greene. Dominant photodetachment channels in $\mathrm{H}^{-}$. PRL, 65:313-16, 1990. Theory - Keys : OU - Species : $\mathrm{H}^{-}$.

[355] C.H. Greene and L. Kim. Two-electron excitations in atomic calcium. I. PRA, 36:2706-17, 1987. Theory Keys : AI - Species : Ca.

[356] C.H. Greene and L. Kim. Streamlined eigenchannel treatment of open-shell spectra. PRA, 38:5953-6, 1988. Theory - Keys : oU - Species : Si.

[357] C.H. Greene and N. Rouze. Interpretation of ascillatory structures in Rb- photodetachment. ZPD, 9:219-21, 1988. Theory - Keys : SF - Species : Rb-.

[358] C.H. Greene and C.E. Theodosiou. Photoionization of the Ba 6s6p ${ }^{1} \mathrm{P}_{1}$ state. PRA, 42:5773-5, 1990. Theory Keys : ou - Species : Ba.

[359] P.T. Greenland, D.N. Travis and D.J.H. Wort. Resonant ionization polarisation spectroscopy in uranium. JPB, 23:2945-56, 1990. Exp-Keys : FP - Species : U.

[360] U. Griesmann, N. Shen, J.P. Connerade, K. Sommer and J. Hormes. New measurements of the cross sections of doubly excited resonances in calcium. JPB, 21:L83-8, 1987. ExP-Keys : OU AI - Species : Ca.

[361] J. Grochmalicki, J.P. Kuklinski and M. Lewenstein. Above-threshold jonisation and electron scattering in intense laser fields. JPB, 19:3649-68, 1986. Theory-Keys : MP - Species : Xe.

[362] J. Grochmalicki, M. Lewenstein, M. Wilkans and K. Rzazewski. Beyond above-threshold-ionization : ionization of an atom by an ultrashort laser pulse above atomic intensity. JOSAB, 7:607-16, 1990. Theory - Keys : MP General.

[363] J. Grochmalicki, J. Mostowski and M. Trippenbach. Above-threshold ionisation of the classical atom. JPB, 21:1673-80, 1988. Theory - Keys : MP - Species : H.

[364] D.S. Guo and T. Åberg. Quantum electrodynamical approach to multiphoton ionisation in the high-intensity H field. JPA, 21:4577-91, 1988. Theory - Keys : MP - General.

[365] D.S. Guo, T. Åberg and B. Crasemann. Scattering theory of multiphoton ionization in strong fields. PRA, 40:4997-5005, 1989. Theory - Keys : MP - General.

[366] S.L. Haan, M. Walhout and J. Cooper. Threshold effects in photodetachment near an autodetaching or excitedstate resonance. PRA, 41:2598-609, 1990. Theory - Keys : OU AI - General.

[367] W. Habenicht, K. Muller-Dethlefs and E.W. Schlag. Zero-kinetic-energy photoelectron-photoion co ncidence measurements of $\mathrm{Ar}$ in the $2 p$ and $\mathrm{N}_{2}$ in the 1s region. JESRP, 52:697-710, 1990. Exp - Keys : IN - Species : Ar.

[368] H. Haberland and M. Oschwald. Two-photon ionisation of metastable helium : complex structure in the 2S-3P resonance profiles. JPB, 21:1183-93, 1988. Exp-Keys : AI FP - Species : He. 
[369| R.I. Hall, L. Avaldi, G. Dawber, P.M. Rutter, M.A. Mac Donald and G.C. King. Observation of the argol photoelectron satellites near threshold. JPB, 22:3205-16, 1989. Exp-Keys : OU IN - Species : Ar.

[370] R.I. Hall, L. Avaldi, G. Dawber, M. Zubek and G.C. King. Observation of the krypton and xeon photoelectrol sattelite spectra near threshold. JPB, 23:4469-85, 1990. Exp-Keys : OU IN - Species : Kr, Xe.

[371] P. Hamacher and J. Hinze. The variational $R$-matrix method : resonances in the photoionisation of He fo photon energies 58-65 eV. JPB, 22:3397-410, 1989. Theory-Keys : OU AI - Species : He.

[372] C.S. Han. Dynamics of the above-threshold ionization of atoms in an intense field. JPB, 23:L495-500, 1990 Theory - Keys : MP - General.

[373] C.S. Han and R. Shakeshaft. Monte Carlo integration of multiphoton ionization matrix elements in the weak-fielc regime. PRA, 39:4278-80, 1989. Theory-Keys : FP - Species : H.

[374] D. Handschuh, M. Meyer, M. Pahler, T. Prescher, M. Richter, B. Sonntag and H.E. Wetzel. Partial and tota photoionization cross sections of atomic $\mathrm{Ba}, \mathrm{La}$ and $\mathrm{Ce}$ in the range of the giant $4 \mathrm{~d}$ resonances. JPCo, 48C9:539-42, 1987. Exp-Keys : 00-Species : Ba, La, Ce.

[375] J.E. Hansen, J. Brilly, E.T. Kennedy and G. O'Sullivan. Rise and Fall of the $4 d^{10} \rightarrow 4 d^{9} 4 f$ resonance in the $X_{6}$ isoelectronic sequence. PRL, 63:1934-7, 1989. Exp-Keys : OU -Species : $\mathrm{Ba}^{2+}, \mathrm{La}^{3+}$.

[376] D. Hanstorp, C. Bengtsson and D.J. Larson. Angular distributions in photodetachment of O- PRA, 40:670-75 1989. Exp - Keys : OU - Species : $\mathrm{O}^{-}$.

[377] D. Hanstorp, P. Devynck, W.G. Graham and J.R. Peterson. Observation of metastable autodetaching CaPRL, 63:368-71, 1989. Exp-Keys : AI - Species : $\mathrm{Ca}^{-}$.

[378] P.G. Harris, H.C. Bryant, A.H. Mohagheghi, R.A. Reeder, C.Y. Tang, J.B. Donahue and C.R. Quick. Observation of doubly excited resonances in the $\mathrm{H}^{-}$ion. PRA, 42:6443-65, 1990. Exp - Keys : AI - Species $\mathrm{H}^{-}$.

[379] H.K. Haugen and A.S. Othonos. Fluorescence studies of multiple-photon ionization processes : four and fivephoton ionization of $\mathrm{Sr}$ at wavelengths of 558-590 nm. PRA, 39:3392, 1989. Exp - Keys : FP - Species . Sr.

[380] A. Hausmann, B. Kämmerling, H. Kossmann and V. Schmidt. New approach for a perfect experiment : 2p photoionization in atomic magnesium. PRL, 61:2669-72, 1988. Exp - Keys : IN - Species : Mg.

[381] T. Hayaishi, E. Murakami, A. Yagishita, F. Koike, Y. Morioka and J.E. Hansen. Photoion spectra in coincidence with threshold electrons near the Ar 2p ionisation limits. JPB, 21:3203-9, 1988. Exp - Keys : IN - Species : Ar.

[382] T. Hayaishi, M. Watanabe, A. Yagishita and Nakamura M. Multiple photoionization from 3p excitation of Kr and 4p excitation of Xe. ZPD, 4:25-30, 1987. Exp-Keys : MI - Species : Kr, Xe.

[383] T. Hayaishi, A. Yagishita, E. Shigemasa, E. Murakami and Y. Morioka. Coicidence spectra of threshold electrons and ions around the $\mathrm{Kr} 3 \mathrm{~d}$ and Xe $4 \mathrm{~d}$ delayed onset regions. PS, 41:35-7, 1990. Exp - Keys : IN - Species : $\mathrm{Kr}, \mathrm{Xe}$.

[384] T. Hayaishi, A. Yagishita, E. Murakami, E. Shigemasa, Y. Mirioka and T. Sasaki. Photoion spectra in coincidence with threshold electrons near the Kr $3 \mathrm{~d}$ ionisation limits. JPB, 23:1633-9, 1990. Exp - Keys : IN - Specjes : Kr.

[385] T. Hayaishi, A. Yagishita, E. Shigemasa, E. Murakami and Y. Morioka. Photoion spectra in coincidence with threshold electrons near the Xe $4 \mathrm{~d}$ ionization limits. JPB, 23:4431-9, 1990. Exp - Keys : IN - Species : Xe.

[386] M.A. Hayes and M.P. Scott. Resonances in the simultaneous photoionisation and excitation of He. JPB, 21:1499-507, 1988. Theory - Keys : OU AI - Species : He.

[387] P.A. Heimann, U. Becker, H.G. Kerkhoff, B. Langer, D. Szostak, R. Wehlitz, D.W. Lindle, T.A. Ferrett and D.A. Shirley. Helium and neon photoelectron satellites at threshold. PRA, 34:3782-91, 1986. Exp - Keys: OU - Species: $\mathrm{He}$, Ne. 
[388] P.A. Heimann, D.W. Lindle, T.A. Ferrett, S.H. Liu, L.J. Medhurst, M.N. Piancastelli, D.A. Shirley, U. Becker, H.G. Kerkhoff, B. Langer, D. Szostak, and R. Wehlitz. Shake-off on inner-shell resonances of Ar, Kr and Xe. JPB, 20:5005-22, 1987. Exp-Keys : IN - Species : Ar, Kr, Xe.

[389] K. Helenelund, U. Gelius, P. Froelich and O. Goscinski. Photon-induced post-collisional Auger decay : a modified 'shake-down' model. JPB, 19:379-86, 1986. Theory - Keys : IN - General.

[390] H. Hieronymus, M. Khol, J. Neukammer, A. Konig, H. Rinneberg and H. Spinger-Bolk. Perturbed angular distribution of electrons following photoionization of $6 s n s{ }^{1} \mathrm{~S}_{0}$ barium Rydberg states. PRA, 41:1477-91, 1990. Exp - Keys : OU - Species : Ba.

[391] R. Hippler, H. Schwier, S. Jetzke and H.O. Lutz. Photoelectron-spectroscopy of multi-photon ionization of rare gases with circularly and linearly polarized light. ZPD, 11:199, 1989. Exp-Keys : MP - Species : Ar, Kr, Xe.

[392] Y.K. Ho. Resonances in helium atoms associated with the $N=4$ and $N=5 \mathrm{He}^{+}$thresholds. ZPD, 11:277, 1989. Theory - Keys : AI - Species : He.

[393] Y.K. Ho. Resonances in $\mathrm{H}^{-}$associated with the $N=7$ hydrogen threshold. PRA, 41:1492-5, 1990. Theory Keys : AI - Species : $\mathrm{H}^{-}$.

[394] W. Hogervorst, E.A.J.M. Bente, R.J. de Graaff, C.T.W. Lahaije and W. Vassen. Laser spectroscopy of bound and autoionizing Rydberg series of two-electron atoms. PS, T26:9, 1989. Exp-Keys : AI - Species: He, Ba ${ }^{54+}$.

[395] E. Hollauer and M.A.C. Nascimento. Photoionization cross sections and dynamic polarizabilities for the lithium atom and positive ion using $L^{2}$ basis sets and correlated wave functions. PRA, 42:6608-15, 1990. Theory Keys : OU - Species : $\mathrm{Li}^{+}, \mathrm{Li}$.

[396] J. Hormes, K. Sommer, U. Kuetgens, W. Dussa and R. Chauvistre. High resolution spectroscopy of atoms and molecules using synchrotron radiation : atomic mercury and mercury halides. PS, 37:694-8, 1988. Exp - Keys : OU - Species : $\mathrm{Hg}$.

[397] M. Hou, P. Breger, G. Petite and P. Agostini. Direct multiphoton transitions to highly excited two-electron states. JPB, 23:L583-9, 1990. Exp-Keys : MP - Species : Mg.

[398] M. Hou, Z. Zhang, J. Li, L. Liu and X. Ming. Absolute measurement of photoionization cross-sections of excited states $3 s 5 s^{1} \mathrm{~S}$ and $3 \mathrm{~s} 4 \mathrm{~d}^{1} \mathrm{D}$ in magnesium. ChPL, 6:60-3, 1989. Exp - Keys : OU - Species : $\mathrm{Mg}$.

[399] C.M. Houston, S.L.T. Drysdale, R. Jennings, A.P. Land, K.W.D. Ledingham, R.P. Singhal, M.H.C. Smyth, D.T. Stewart and $M$. Towrie. Two and three-photon ionisation transitions in caesium vapour. JPD, 21:59-62, 1988. Exp - Keys : FP - Species : Cs.

[400] Q. Hu, L. Yin, L. Zhang, G. Zhang, G. Jing, J. Cui, H. Su and H. Lin. Study of three-step photoionization of U atoms using a hollow-cathode discharge tube. ChP, 7:200-4, 1987. Exp-Keys : FP - Species : U.

[401] S. Hu, S. Mei, S. Zhang, X. Chen and Y. Xu. Observation and measurement of the autoionization spectra of atomic samarium. ChPL, 6:64-7, 1989. Exp-Keys : AI - Species : Sm.

[402] S. Hu, S. Zhang, J. Lu and J. Sun. Observation and analysis of the autoionization spectra of Sr. ChP, 7:785-90, 1987. Exp-Keys : AI - Species : Sr.

[403] M. Hube, M. Kumkar, M. Dieckmann, R. Beigang and B. Wellegehausen. Potassium photoionization laserproduced by inner shell ionization of excited potassium. OC, 66:107-10, 1988. Exp - Keys: FP IN - Species : K.

[404] H.J. Humpert, R. Hippler, H. Schwier and H.O. Lutz. Multiphotoionization of xenon. NATO1, p. 649-55, 1986. Exp-Keys : MP - Species : Xe.

[405] M.H.R. Hutchinson and K.M.M. Ness. Laser-induced continuum structure in xenon. PRL, 60:105-7, 1987. Exp - Keys : MP - Species : Xe.

[406] A.K. Ito, K. Ueda, T. Namioka, K. Yoshino and Y. Morioka. High-resolution absorption spectrum of Ne I in the region of 565-595 $\AA$. JOSAB, 5:2006-14, 1988. Exp \& theory - Keys : OU - Species : Ne.

[407] Y. Itoh, T. Hayaishi, Y. Itikawa, T. Koizumi, T. Nagata, Y. Sato, H. Shibata, A. Yagishita and M. Yoshino. Rb $4 p$ resonances studied by a photoion-yield spectrum. JPB, 21:L727-34, 1988. Exp - Keys : AI - Species : Rb. 
[408] D. Janezic, M. Penca, B.B. Lide and D.R. Lide. Graphical handling of wide-ranging data : graphing of photon cross-section data. JCICS, 30:30-2, 1990. Theory - Keys : OU - General.

[409] E. Jannitti, P. Nicolosi and G. Tondello. Photoabsorption spectra of C-ions in the soft X-rays. JPCo, 48C9:239-42, 1987. Exp - Keys : IN - Species : $\mathrm{C}^{4+}$.

[410] E. Jannitti, P. Nicolosi and G. Tondello. Photoionization cross-section measurement of the C V ion. PLA, 131A:186-9, 1988. Exp - Keys : OU - Species : $\mathrm{C}^{4+}$.

[411] E. Jannitti, P. Nicolosi and G. Tondello. Absorption spectra from 1s inner shell electron of ionized and neutral carbon. PS, 41:458-63, 1990. Exp-Keys : IN - Species : $\mathrm{C}^{4+}, \mathrm{C}^{3+}, \mathrm{C}^{2+}, \mathrm{C}$.

[412] B. Jaskolska and W. Woznicki. An attempt to use correlated wavefunctions to determine autoionizing states. I. ${ }^{2} \mathrm{P}^{\circ}$ resonances in the lithium atom. PS, 39:230-3, 1989. Theory - Keys : AI - Species : Li.

[413] B. Jaskolska and W. Woznicki. An attempt to use correlated wavefunctions to determine autoionizing states. II. ${ }^{2} \mathrm{D}$ resonances in the lithium atom. PS, 39:234-7, 1989. Theory - Keys : AI - Species : Li.

[414] J. Javanainen and J.H. Eberly. Numerical experiments on above-threshold ionization. MP, p. 88-96, 1988. Th. review - Keys : MP - General.

[415] J. Javanainen and J.H. Eberly. Model ab initio study of intense laser photoionisation. JPB, 21:L93-8, 1988. Theory -- Keys : MP - General.

[416] J. Javanainen, J.H. Eberly and Q. Su. Numerical simulations of multiphoton ionization and above-threshold electron spectra. PRA, 38:3430-46, 1988. Theory - Keys : MP - General.

[417] J. Javanainen and P.L. Gould. Linear intensity dependence of a two-photon transition rate. PRA, 41:5088-91, 1990. Theory - Keys : FP - Species : Xe.

[418] R.V. Jensen and B. Sundaram. Role of "scars" in the suppression of ionisation in intense high frequency fields. PRL, 65:1964, 1990. Theory - Keys : MP - General.

[419] R.V. Jensen, S.M. Susskind and M.M. Sanders. Microwave ionization of highly excited hydrogen atoms : a test of the correspondence principle. PRL, 62:1476-9, 1989. Theory - Keys : MP - Species : H.

[420] T.F. Jiang and A.F. Starace. Two-photon detachment of the negative chlorine ion. PRA, 38:2347-55, 1988. Theory-Keys : FP - Species : $\mathrm{Cl}^{-}$.

[421] J. Jimenez-Mier, M.O. Krause, P. Gérard, B. Hermsmeier and C.S. Fadley. Partial and total cross sections and multiplet structure in the photoionization of atomic manganese. PRA, 40:3712-20, 1989. Exp - Keys : OU Species : Mn.

[422] J. Jimenez-Mier, C.D. Caldwell and M.O. Krause. Autoionizing resonances 4d $\rightarrow$ nl in cadmium. PRA, 39:95102, 1989. Exp-Keys : AI - Species : Cd.

[423] W. Jitschin, G. Grosse and P. Rohl. Coster-Kronig factor $f_{13}$ of ${ }^{39} Y$ measured with the synchrotron photoionization method. PRA, 39:103-9, 1988. Exp-Keys : IN - Species : Y.

[424] T.J. John. Continuous absorption by the negative hydrogen ion reconsidered. AA, 193:189-92, 1988. Theory Keys : OU - Species : $\mathrm{H}^{-}$.

[425] R.R. Jones, C.J. Dai and T.F. Gallagher. Ba 6 $\mathrm{p}_{j} n f_{j}^{\prime}$ autoionizing series. PRA, 41:316-26, 1990. Exp - Keys : AI - Species : Ba.

[426] R.R. Jones and T.F. Gallagher. Autoionization of high-l Ba 6 $\mathrm{p}_{1 / 2}$ nl states. PRA, 38:2846-53, 1988. Exp Keys : AI - Species: Ba.

[427] R.R. Jones and T.F. Gallagher. Autoionization of $\mathrm{Ba} 6 \mathrm{p}_{1 / 2}$ nk states in static and microwave fields below the Inglis-Teller limit. PRA, 39:4583-90, 1989. Exp-Keys : AI SF MP - Species : Ba.

[428] R.R. Jones and T.F. Gallagher. Experimental observation of angular correlations between Rydberg electrons in the Ba n'l'nl autoionizing series. PRA, 42:2655-60, 1990. Exp-Keys : OU AI - Specjes : Ba.

[429] R.R. Jones, Y. Zhu, U. Eichmann and T.F. Gallagher. Multiphoton double ionization of barium. IEEETPS, 16:534-40, 1988. Exp - Keys : MP MI - Species : Ba. 
[430] A. Joshi and R.R. Puri. Effects of atomic coherence on a Rydberg atom undergoing a two-photon transition in a lossless cavity. OC, 75:189-96, 1990. Exp-Keys : FP - General.

[431] Y.N. Joshi and M. Mazzoni. Photoabsorption of atomic tellurium : inner d-shell excitation. JPB, 21:1167-22, 1988. Exp - Keys : IN - Species : Te.

[432] Y.N. Joshi and M. Mazzoni. 3d-shell excitation in photoabsorption of atomic selenium. CJP, 66:326-9, 1988. Exp - Keys : OU IN - Species : Se.

[433] Y. Justum, A. Maquet and Y. Heno. Gauge invariance and approximate multiphoton calculations in hydrogen. PRA, 41:2791-5, 1990. Theory - Keys : MP - Species : H.

[434] N.M. Kabachnik and O.V. Lee. Spin polarisation of Auger electrons following atomic photoionisation by circularly polarised X-rays. JPB, 22:2705-16, 1989. Theory - Keys : IN - Species : Kr, Xe.

[435] N.M. Kabachnik and I.P. Sazhina. Spin-polarised Auger-electron spectroscopy of the d atomic shell. Model calculation for noble-gas atoms. JPB, 21:267-76, 1988. Theory - Keys : IN - Species : Ar, Kr, Xe.

[436] N.M. Kabachnik, I.P. Sazhina, I.S. Lee and O.V. Lee. The effect of many-electron correlations on the angular distribution and spin polarisation of Auger electrons in $\mathrm{Kr}$ and Xe. JPB, 21:3695-707, 1988. Theory - Keys : IN - Species : $\mathrm{Kr}, \mathrm{Xe}$.

[437] N.M. Kabachnik and I.P. Sazhina. On the problem of a 'complete' experimental characterisation of Auger decay . JPB, 23:L353-7, 1990. Theory - Keys : IN - General.

[438] K.S. Kahlon, H.S. Aulakh, N. Singh, R. Mittal, K.L. Allawadhi and B.S. Sood. Experimental investigation of alignment of the $\mathrm{L}_{3}$ subshell vacancy state produced after photoionization in lead by $59.57 \mathrm{keV}$ photons. JPB, 23:2733-43, 1990. Exp - Keys : IN - Species : Pb.

[439] K.S. Kahlon, K. Shatendra, K.L. Allawadhi and B.S. Sood. Experimental investigation of angular dependence of photon-induced L-shell X-ray emission intensity. Pramana, 35:105-14, 1990. Exp - Keys : IN - Species : $\mathrm{Pb}, \mathrm{U}$.

[440] A. Kallenbach, M. Kock and G. Zierer. Absolute cross sections for photoionization of laser-excited Ba I states measured on a thermionic diode. PRA, 38:2356-60, 1988. Exp-Keys : FP OU - Species : Ba.

[441] E. Kallne, J. Kallne, J. Dubau, E.S. Marmas and J.E. Rice. X-ray satellite lines of high-n Rydberg transitions in $\mathrm{Ar}^{16 \div}$. PRA, 38:2056-65, 1988. Exp-Keys : IN - Species : $\mathrm{Ar}^{16+}$

[442] P. Kalman. X-ray absorption by atoms under intense laser fields. PRA, 39:2428, 1989. Theory - Keys : MP IN - General.

443] P. Kalman. Hindering effect of an intense laser field in near-absorption-edge laser-assisted X-ray absorption. PRA, 39:3200-3, 1989. Theory - Keys : MP IN - General.

[444] J.Z. Kaminski. 1/n expansion for $n$-photon processes. Quasiclassical approach. PS, 42:417-23, 1990. Theory Keys : MP - General.

[445] B. Kämmerling, H. Kossman and V. Schmidt. 4d photoionisation in xenon : absolute partial cross section and relative strength of $4 \mathrm{~d}$ many-electron processes. JPB, 22:841-54, 1989. Exp - Keys : IN - Species : Xe.

[446] B. Kämmerling, B. Krassig and V. Schmidt. Connection between the angular distribution of Auger electrons and spectator autoionization electrons following $4 \mathrm{~d}$ ionization/excitation in xenon. JPB, 23:4487-4503, 1990. Exp - Keys : IN - Species : Xe.

[447] B. Kämmerling, V. Schmidt, W. Melhorn, W.B. Peatman, F. Schaefers and T. Schroeter. Auger decay parameters $\alpha_{2}$ for Krypton $\mathrm{M}_{45}-\mathrm{N}_{23} \mathrm{~N}_{23}$ transitions. JPB, 22:597-602, 1989. Exp - Keys : IN - Species : Kr.

[448] A.V. Kapliauskene. Doubly excited states of $\mathrm{Ne}^{2+}, \mathrm{Ne}^{3+}$ and $\mathrm{Ne}^{4+}$. SPC, 28:93-4, 1989. Theory - Keys : AI Species : $\mathrm{Ne}^{4+}, \mathrm{Ne}^{3+}, \mathrm{Ne}^{2+}$.

[449] K.R. Karim and C.P. Bhalla. $Z$ dependence of atomic parameters for selected autoionizing states of two-electron ions. PRA, 37:1507-11, 1988. Theory-Keys : AI - Species : He seq with $10 \leq Z \leq 28\left(\mathrm{Ne}^{8+}, \mathrm{Si}^{12+}, \mathrm{Ar}^{16+}\right.$, $\left.\mathrm{Ca}^{18+}, \mathrm{Ti}^{20+}, \mathrm{Cr}^{22+}, \mathrm{Fe}^{24+}, \mathrm{Ni}^{26+}\right)$. 
[450] K.R. Karim and C.P. Bhalla. Auger and radiative deexcitation rates and energies of $3 \mathrm{~d} \mathbf{3} \mathrm{d}^{\prime}$ states of helium-like argon and silicon. PS, 38:795-801, 1988. Theory-Keys : IN AI - Specjes : $\mathrm{Si}^{12+}, \mathrm{Ar}^{16+}$.

[451] A. Karosiene and A. Kancerevicius. On the investigation of the free electron photoionization cross section in the case of the well effective potential. SPC, 24:28-34, 1986. Theory - Keys : OU - General.

[452] E. Karule. Analytical continuation of the transition matrix elements and multiphoton above-threshold ionisation of atomic hydrogen. JPB, 21:1997-2014, 1988. Theory - Keys : MP - Species : H.

[453] E. Karule. Above-threshold ionization of atomic hydrogen. JOSAB, 7:631-8, 1990. Theory - Keys : MP Species: $\mathrm{H}$.

[454] A. Kassaee, M.L. Rustgi and S.A.T. Long. Two-photon ionisation of atomic hydrogen with elliptically polarized . light. PRA, 37:999-1002, 1988. Theory-Keys : FP - Species : H.

[455] H. Kawakami, S. Kato, F. Naito, K. Nisimura, T. Ohshima, S. Shibata, T. Suzuki, K. Ukai, N. Morikawa, N. Nogawa, T. Nagafuchi, H. Taketani, M. Iwahashi, K. Isatake, Y. Fukushima, T. Matsuga and T. Taniguchi. Precise measurement of Ag KLM and KLN Auger spectra. PLA, 127A:175-8, 1988. Exp-Keys : IN - Species : Ag.

[456] R.D. Kay, B.H. Houston and J.P. Czechanski. Two-photon ionisation of $2^{1} \mathrm{~S}$ metastable helium. JPB, 22:L10713, 1989. Exp - Keys : FP - Species : He.

[457] A.K. Kazanskii and V.N. Ostrovskii. The Rydberg series for the doubly excited states of the helium atom. SPJETP, 65:281-6, 1987. Theory - Keys : AI - Species : He.

[458] H.P. Kelly. Many body calculations of photoionization cross sections. PS, T17:109-19, 1987. Th. review - Keys : OU - Species : $\mathrm{He}, \mathrm{Cl}, \mathrm{Ca}, \mathrm{Mn}, \mathrm{Cd}, \mathrm{Xe}, \mathrm{Eu}$.

[459] J.F. Kelly, J.P. Hessler and G. Alber. Experimental studies of three-photon ionization of Ba : evidence of channel interference and Raman coupling. PRA, 33:3913-37, 1986. Exp-Keys : FP - Species : Ba.

[460] C. Kerling, N. Böwering and U. Heinzmann. Photoelectron angular distributions from laser-excited aligned $\mathrm{Yb}$ atoms ionized by vacuum ultraviolet radiation. JPB, 23:L629-35, 1990. Exp - Keys : FP OU - Species : Yb.

[461] M.A. Khan, E.E. Khawaja and H.A. Al-Juwair. The ionization of calcium atoms through resonant laser pumping of ${ }^{3}$ P metastable states. JPB, 23:L533-9, 1990. Exp - Keys : MP - Species : Ca.

[462] D. Kim, S. Fournier, M. Saeed and L.F. DiMauro. Magnesium atoms in an intense nonresonant laser field. PRA, 41:4966-73, 1990. Exp - Keys : MP - Species : Mg.

[463] L. Kim and C.H. Greene. Two-electron excitations in atomic calcium. II. Fine-structure effects. PRA, 36:4272-9, 1987. Theory-Keys : OU AI - Species : Ca.

[464] L. Kim and C.H. Greene. Two-electron excitations in atomic calcium. III. High-lying resonances above the 4p threshold. PRA, 38:2361-70, 1988. Theory-Keys : OU AI - Species : Ca.

[465] Y.S. Kim. Angular momentum transfer formalism for photoionisation of excited rare gas atoms. PRA, 37:223740, 1988. Theory - Keys : OU - General.

[466] G.C. King, M. Zubek, P.M. Rutter, F.H. Read, A.A. MacDowell, J.B. West and D.M.P. Holland. A study of threshold photoionisation and double photoionisation in helium. JPB, 21:L403-8, 1988. Exp - Keys : OU MI Species : He.

[467] I.Y. Kiyan and V.P. Krainov. Resonant and virtual processes of above-threshold ionization of negative ions. SPJETP, 69:909-12, 1990. Theory - Keys : MP - General.

[468] A.V. Klarenbosch, K.K. Geerinck, T.O. Klaassen and W.T. Wenckebach. Ionization energies and lifetime broadening of autoionizing states of the hydrogen atom in strong magnetic fields: theory vs. experiment. EL, 13:237-42, 1990. Exp \& theory - Keys : SF AI - Species : H.

[469] P.L. Knight, M.A. Lauder and B.J. Dalton. Laser-induced continuum structure. PRp, 190:1-61, 1990. Review - Keys : MP - General.

[470] P.M. Koch, L. Moorman, B.E. Sauer, E.J. Galvez, K.A.H. van Leeuven and D. Richards. Experiments in quantum chaos : microwave ionization of hydrogen. PS, T26:51, 1989. Exp-Keys : MP - Species : H. 
[471] T. Koizumi, T. Hayaishi, T. Matsuo, K. Shima, H. 'Tawara, T. Tonuma and A. Yagishita. Photoionization cross sections of xenon in the L edge region. JPSJ, 58:13-16, 1989. Exp-Keys : IN - Species : Xe.

[472] T. Koizumi, T. Hayaishi, Y. Itikawa, Y. Itoh, T. Matsuo, Y. Sato, E. Shigemasa, A. Yagishita and M. Yoshino. Multiply charged ions of Rb and Sr produced by 3d-shell photoionisation. JPB, 23:403-15, 1990. Exp-Keys : IN MI - Species: Rb, Sr.

[473] K. Kokubun, H. Hashizume, H. Shimizu, Y. Oowadano, Y. Matsumoto and K. Endo. Non resonant multiphoton ionisation of rare gases. JVSTA, 8:3310-3, 1990. Exp-Keys : MP - Species : Ar, Kr, Xe.

[474] K. Kokubun, S. Ichimura and H. Shimizu. Multiphoton ionization of Xe and $\mathrm{Kr}$ atoms by an ArF excimer laser. JJAP2L, 29:L1509-10, 1990. Exp-Keys : MP - Species : Kr, Xe.

[475] K. Kokubun, S. Ichimura and H. Shimizu. Nonresonant multiphoton ionization of He and Ne atoms. JJAP2L, 29:673-4, 1990. Exp - Keys : MP - Species : He, Ne.

[476] M. Kompitsas, S. Cohen, C.A. Nicolaides, O. Robaux, M. Aymar and P. Camus. Observation and theoretical analysis of the odd $J=3$ autoionizing spectrum of $\mathrm{Sr}$ up to the $4 \mathrm{~d}$ threshold. JPB, 23:2247-67, 1990. Exp \& theory - Keys : AI - Species : Sr.

[477] V.D. Kondratovich and V.N. Ostrovsky. Resonance and interference phenomena in the photoionisation of a hydrogen atom in a uniform electric field.III. Comparison with recent experimental and theoretical results. JPB, 23:21-43, 1990. Exp \& theory - Keys : SF - Species : H.

[478] V.D. Kondratovich and V.N. Ostrovsky. Resonance and interference pbenomena in the photoionization of a hydrogen atom in a uniform electric field : IV. Differential cross sections. JPB, 23:3785-3809, 1990. Theory Keys : $\mathbf{5 F}$ - Species : $\mathrm{H}$.

[479] H. Kossmann, B. Krassig and V. Schmidt. New determination of Beutler-Fano parameters for the 3s3p ${ }^{1} P_{1}$ resonance in helium. JPB, 21:1489-97, 1988. Exp-Keys : AI - Species : He.

[480] H. Kossmann, V. Schmidt and T. Andersen. Test of Wannier threshold laws : double photoionization cross section in helium. PRL, 60:1266-9, 1988. Exp-Keys: MI - Species : He.

[481] A. Kovalik, V. Brabec, J. Novak, O. Dragoun, V.M. Gorozhankin, A.F. Novgorodov, and Ts. Vylov. The K Auger spectrum of manganese from ${ }^{55} \mathrm{Fe}$ decay. JESRP, 50:89-101, 1990. Exp - Keys : IN - Species : Mn.

[482] G. Kracke, H. Marxer, J.T. Broad and J.S. Briggs. Multiphoton ionisation of atomic hydrogen and the angular distribution of photoelectrons. ZPD, 8:103-4, 1988. Exp \& theory - Keys : MP - Species : H.

[483] V.P. Krainov and Z.G. Melikishvili. Dependence of the probability of the direct process of atomic multiphoton ionization on the polarization of the radiation. OS, 65:579-80, 1988. Theory - Keys : MP - General.

[484] V.V. Krasnikov, M.S. Pshenichnikov and V.S. Solomatin. Direct observations on the dynamic Stark effect in two-photon absorption and parametric interaction. MUPB, 43:42-6, 1989. Exp - Keys : FP - Species : Na.

[485] H.F. Krause. Observation of Landau resonances well above threshold in a high-resolution $(\Delta f=70 \mathrm{MHz})$ laser photodetachment study of $\mathrm{O}^{-}$. PRL, 64:1725-8, 1990. Exp-Keys : SF - Species : $\mathrm{O}^{-}$.

[486] M.O. Krause and C.D. Caldwell. Photoionization in the K-shell of atomic beryllium. JPCo, 48-C9:473-8, 1987. Exp - Keys : IN - Species : Be.

[487] M.O. Krause and C.D. Caldwell. Strong correlation and alignment near the Be 1 photoionization threshold. PRL, 59:2736-9, 1987. Theory - Keys : IN - Species : Be.

[488] M.O. Krause, A. Svensson, A. Fahlman and T.A. Carlson. Autoionization resonances in atomic $\mathrm{Ga}$, In and $\mathrm{Pb}$. ZPD, 2:327-35, 1986. Exp - Keys : AI - Species : Ga, In, Pb.

[489] S. Kroll and W.K. Bischel. Two-photon absorption and photoionization cross-section measurements in the $5 p^{5} 6 p$ configuration of xenon. PRA, 41:1340-9, 1990. Exp - Keys : FP - Species : Xe.

[490] P. Krstic and M.H. Mittleman. $S$-matrix theory of multiphoton ionization. JOSAB, 7:587-91, 1990. Theory Keys : MP - General.

[491] Y. Kuang, Q. Dong and R.H. Pratt. Photoionisation from ions : dependence on the degree of ionization. ChJP, 27:335-50, 1989. Theory - Keys : OU - Species : Ne ions. 
[492] J.R. Kuklinski and M. Lewenstein. Pulse-shape-induced phenomena in photodetachment. JPB, 20:1387-98, 1987. Theory - Keys : OU - General.

[493] K.C. Kulander. Theory of multiphoton ionization of atoms by strong, short pulsed lasers. MP, p. 116-23, 1988. Theory - Keys : MP - Species : He.

[494] K.C. Kulander. Time-dependent theory of multiphoton ionization of xenon. PRA, 38:778-87, 1988. Theory Keys : MP - Species : Xe.

[495] Sh.D. Kunikeev and V.S. Senanshenko. Effect of post-collision interaction on the interference of autoionization resonances with a continuum spectrum. MUPB, 44:18-21, 1989. Theory - Keys : IN AI - General.

[496] J. Kupersztych. The decoupling problem in intense-field ionization. PS, 42:51-7, 1990. Theory - Keys : MP General.

[497] A.V. Kuplyauskene. Autoionization energies of doubly excited states of $\mathrm{Ne}^{2+} . \mathrm{OS}, 66: 299-301,1989$. Theory Keys : AI - Species : $\mathrm{Ne}^{2+}$.

[498] M. Kutzner, Z. Altun and H.P. Kelly. Photoionisation of atomic barium including effects of relaxation and polarisation. PRA, 41:3612-23, 1990. Theory-Keys : OU - Species : Ba.

[499] M. Kutzner, V. Radojevic and H.P. Kelly. Extended photoionization calculations for xenon. PRA, 40:5052-7, 1989. Theory - Keys : OU - Species : Xe.

[500] N. Kwon, P.S. Armstrong, T. Olsson, R. Trainham and D.J. Larson. Single and multiphoton detachment from stored $F^{-}$ions. PRA, 40:676-82, 1989. Exp-Keys : MP OU - Species : F-.

[501] A. L'Huillier. Multiple ionization of atoms through multiphoton absorption. JPCo, 48-C9:415-25, 1987. Review - Keys : MP MI - Species : Sr, Xe.

[502] A. L'Huillier, L. Jonsson and G. Wendin. Many-electron effects in multiphoton ionization : screening effects in single-electron ionization. PRA, 33:3938-55, 1986. Theory - Keys : MP - Species : He.

[503] A. L'Huillier, L.A. Lompré, M. Ferray and G. Mainfray. Generation of very high harmonics of optical radiation in rare gases. AIPCP, 205:505-12, 1990. Exp - Keys : MP - Species : Xe.

[504] A. L'Huillier and G. Wendin. A many-body approach to two- and three-photon double ionisation and excitation of xenon. JPCo, 48-C9:483-6, 1987. Th. review - Keys : FP MI - Species : Xe.

[505] A. L'Huillier and G. Wendin. Many-body approach to two- and three-photon double ionization and excitation of xenon. PRA, 36:5632-44, 1987. Theory - Keys : FP MI - Species : Xe.

[506] A. L'Huillier and G. Wendin. Two-photon electron detachment of negative iodine. JPB, 21:L247-53, 1988. Theory - Keys : FP - Species : $\mathrm{I}^{-}$.

[507] A. Laakkonen and G. Graeffe. M X-ray linewidths of gold. JPCo, 48-C9:605-8, 1987. Exp - Keys : IN Species : Au.

[508] P. Lablanquie, J.H.D. Eland, I. Nenner, P. Morin, J. Delwiche and M.J. Hubin-Franskin. Threshold behavior in single-photon double ionization of argon. PRL, 58:992-5, 1987. Exp - Keys : MI - Species : Ar.

[509] P. Lablanquie, K. Ito, P. Morin, I. Nenner and J.H.D. Eland. One photon double ionization of helium : threshold behaviour. ZPD, 16:77-9, 1990. Exp-Keys : MI - Species : He.

[510] K.J. LaGattuta. Laser effects in photoionization : numerical solution for a one-dimensional $\delta$ potential. PRA, 40:683-95, 1989. Theory - Keys : MP - General.

[511] K.J. LaGattuta. Laser effects in photoionization : numerical solution of coupled equations for three-dimensional Coulomb potential. JOSAB, 7:639-46, 1990. Theory - Keys : MP - General.

[512] K.J. LaGattuta. Laser effects in photoionisation. II. Numerical solution of coupled equations for atomic hydrogen. PRA, 41:5110-6, 1990. Theory - Keys : MP - Species : H.

[513] J. Lahiri and S.T. Manson. Photoionisation of excited $n$ f states in the Cs isoelectronic sequence : a new example of orbital collapse. PRA, 37:1047-9, 1988. Theory-Keys : OU - Species : Cs seq with $55 \leq Z \leq 65$ (Cs, Ba ${ }^{+}$, $\left.\mathrm{La}^{2+}, \mathrm{Ce}^{3+}, \mathrm{Pr}^{4+}, \mathrm{Nd}^{5+}, \mathrm{Pm}^{6+}, \mathrm{Sm}^{7+}, \mathrm{Eu}^{8+}, \mathrm{Gd}^{9+}, \mathrm{Tb}^{10+}\right\}$. 
[514] J. Lahiri and S.T. Manson. Observations of excited state photoionization of xenon. AIPCP, 191:469-71, 1989. Theory-Keys : OU - Species: Xe.

[515] P. Lambropoulos, X. Tang, P. Agostini, G. Petite and A. L'Huillier. Multiphoton spectroscopy of doubly excited, bound and autoionizing states of strontium. PRA, 38:6165-79, 1988. Exp \& theory - Keys : AI FP - Species : Sr.

[516] P. Lambropoulos and X. Tang. Multiple excitation and ionization of atoms by strong lasers. JOSAB, 4:821-32, 1987. Review - Keys : MP MI AI - Species : H, Xe.

[517] P. Lambropoulos. Atomic processes under strong electromagnetic fields. NATO1, p. 295-41, 1986. Review Keys : MP - General.

[518] P. Lambropoulos. Multiple excitation and ionization of atoms by strong pulsed lasers. CAMP, 2O:199-216, 1987. Exp. review - Keys : MP MI AI - General.

[519] P. Lambropoulos. Comments on major problems in multiphoton processes. MP, p. 350-6, 1988. Review - Keys : MP - General.

[520] O.L. Landen, M.D. Perry and E.M. Campbell. Resonant multiphoton ionization of krypton by intense UV laser radiation. PRL, 59:2558-61, 1987. Exp-Keys : MP - Species : Kr.

[521] V. Lange, U. Eichmann and W. Sandner. Laser-excited autoionising $\left(4 p_{1 / 2,3 / 2}\right.$ ns) $J=1$ Rydberg series in calcium. JPB, 22:L245-50, 1989. Exp-Keys : AI - Species : Ca.

[522] D.J. Larson, P.S. Armstrong, M.C. Baruch, T.F. Gallagher and T. Olsson. Photodetachment in strong oscillating fields. AIPCP, 205:513-8, 1990. Exp-Keys : MP - Species : $\mathrm{Cl}^{-}$.

[523] D.J. Larson, N. Kwon, P.S. Armstrong, T. Olsson and R. Trainham. Photodetachment of negative ions in strong laser fields. NIMPR, B40-41:248-51, 1989. Exp-Keys : MP - Species : F-, $\mathrm{Cl}^{-}$.

[524] S.V. Lavrentev, V.L. Sukhorukov, A.N. Khoperskii and I.D. Petrov. Radial and angular rearrangement of electron shells on $\mathrm{L}_{1}$ photoionization of Ar. OS, 62:278-9, 1987. Theory - Keys : IN - Species : Ar.

[525] S. Legowski and J. Zaremba. Photoionization interaction of atoms with strong fields. PS, 37:710-19, 1988. Review - Keys : MP - General.

[526] C. Leone, S. Bivona, R. Burion and G. Ferrante. Two-frequency multiphoton ionization of hydrogen atoms. PRA, 38:5642-51, 1988. Theory - Keys : MP - Species : H.

[527] C. Leone, S. Bivona, R. Burlon and G. Ferrante. Multiphoton ionization of atoms by two radiation fields : theory and calculations for hydrogen. JMO, 36:909-23, 1989. Theory-Keys : MP - Species : H.

[528] C. Leone, S. Bivona, R. Burlon, F. Morales and G. Ferrante. Gauge aspects in multichannel multiphoton ionization. PRA, 40:1828-37, 1989. Theory - Keys : MP - Species : H.

[529] C. Leone, R. Burlon, F. Trombetta, S. Basile and G. Ferrante. Strong-field multiphoton ionization of hydrogen. The $S$-matrix treatment of the elementary process. NCD, 9D:609-31, 1987. Theory - Keys : MP - Species : H.

[530] W. Leonski, Tanas R. and S. Kielich. Laser-induced autoionization from a double Fano system. JOSAB, 4:72-7, 1987. Theory - Keys : AI FP - General.

531) N.A. Letyaev and S.I. Starkhova. Angular distribution of photoelectrons in the resonance ionization of helium and helium-like lithium to the $n=2$ states of residual ions. PLA, 126A:8-10, 1987. Theory - Keys : OU - Species : $\mathrm{He}, \mathrm{Li}^{+}$

[532] G. Leuchs and S.J. Smith. Photoelectron angular distributions in resonant multiphoton ionization of atoms. JSLR, 6:296-303, 1986. Exp. review - Keys : MP - General.

[533] J.C. Levin. Synchrotron-radiation experiments with recoil ions. AIPCP, 205:176-83, 1989. Exp. review - Keys : QU - Spccies : Ar ions, Kr ions, Xe ions.

[534] L. Li, M. Wu and P.M. Johnson. One and two-color multiphoton ionization of argon. JCP, 89:3470-8, 1988. Exp - Keys : MP - Species : Ar.

[535] S.F. Li and F.C. Lin. Effect of the multiphoton process and spontaneous emission on laser induced autoionisation. JPB, 22:1183, 1989. Theory - Keys : AI MP - General. 
[536] J.K. Liakos and M. Horbatsch. Time-dependent variational calculation of multiphoton ionization of $H$ atoms in intense laser fields. JOSAB, 7:685-8, 1990. Theory - Keys : MP - Species : H.

[537] S.L. Lin, W. Xiong and P. Lavigne. Creation of multiple charges (up to $\mathrm{Xe}^{6+}$ ) from $\mathrm{Xe}$ atoms by an intense $\mathrm{CO}_{2}$ laser. JOSAB, 4:853-5, 1987. Exp - Keys : MP MI - Species : Xe.

[538] D.W. Lindle. Photoionisation studies of atoms and molecules using synchrotron radiation. NIMPR, A280:161-6, 1989. Exp. review - Keys : oU - General.

[539] D.W. Lindle, L.J. Medhurst, T.A. Ferrett, P.A. Heimann, M.N. Piancastelli, S.H. Liu, D.A. Shirley, T.A. Carlson, P.C. Deshmukh, G. Nasreen and S.T. Manson. Angle-resolved photoemission from the Ar 2p subshell. PRA, 38:2371-4, 1988. Exp \& theory - Keys : IN - Species : Ar.

[540] A. Lisini, P.G. Burke and A. Hibbert. Theoretical study of the core photoionization of lithium. JPB, 23:3767-76, 1990. Theory - Keys : IN - Species : Li.

[541] L. Liu, X.M. Tong and J.M. Li. Photoionization of ionized iron atoms. APS, 37:1800-6, 1989. Theory - Keys : ou - Species : Fe ions.

[542] B. Lohmann. Analysis and model calculations on the angular distribution and spin polarization of Auger electrons. JPB, 23:3147-65, 1990. Theory - Keys : IN - Species : Ba.

[543] T.S. Luk, T. Graber, H. Jara, U. Johann, K. Boyer and C.K. Rhodes. Subpicasecond ultraviolet multiphoton electron spectroscopy of rare gases. JOSAB, 4:847-51, 1987. Review - Keys : MP - Species : He, Ar.

[544] T.M. Luke, J.D. Talman, H. Aksela and M. Levasalmi. Low-order perturbation theory for shake-up crosssections in atomic photoionization in a local-potential model. PRA, 41:1350-60, 1990. Theory - Keys : IN Species : Ne.

[545] D. Luo and A.K. Pradhan. Atomic data for opacity calculations. XI. The carbon isoelectronic sequence. JPB, 22:3377-95, 1989. Theory - Keys : OU - Species : C seq with $6 \leq Z \leq 26\left(\mathrm{C}, \mathrm{N}^{+}, \mathrm{O}^{2+}, \mathrm{F}^{3+}, \mathrm{Ne}^{4+}, \mathrm{Na}^{5+}, \mathrm{Mg}^{6+}\right.$, $\left.\mathrm{Al}^{7+}, \mathrm{Si}^{8+}, \mathrm{S}^{10+}, \mathrm{Ar}^{12+}, \mathrm{Ca}^{14+}, \mathrm{Fe}^{20+}\right)$.

[546] D. Luo, A.K. Pradhan, H.E. Saraph, P.J. Storey and Y, Yu. Atomic data for opacity calculations. X. Oscillator strengths and photoionisation cross sections for O III. JPB, 22:389-406, 1989. Theory - Keys : OU - Species : $\mathrm{O}^{2+}$.

[547] I.C. Lyon. Photoionization of positive atomic ions. NATO1, p. 663-9, 1986. Exp. review - Keys : OU - Species : $\mathrm{Ba}^{+}$.

[548] A. Lyras. Rydberg series of autoionizing states in strong laser fields. ZPD, 15:3-12, 1990. Theory - Keys : AI MP - Species : Al.

[549] A. Lyras, D. Bonian, X. Tang, P. Lambropoulos, A. Dodhy, J.A.D. Stockdale, D. Zei and R.N. Compton. MuItiphoton ionization of cesium via quadrupole transitions : photoelectron angular distributions and polarization effects on total ionization rates. PRA, 37:403-11, 1988. Exp - Keys : FP - Species : Cs.

[550] A. Macias, F. Martin, A. Riera and M. Yanez. 'Simple discretization method for autoionization widths. I. Theory. PRA, 36:4179-86, 1987. Theory - Keys : AI - General.

[551] A. Macias, F. Martin, A. Riera and M. Yanez. Simple discretization method for autoionization widths. II. Atoms. PRA, 36:4187-202, 1987. Theory-Keys : AI - Species : $\mathrm{He}, \mathrm{Li}^{+}, \mathrm{Be}^{2+}, \mathrm{B}^{3+}$.

[552] A. Macias and A. Riera. Calculation of resonance widths of ${ }^{3} \mathrm{P}$ states in He-like systems. JPB, 21:1-13, 1987. Theory-Keys : AI - Species : He seq with $2 \leq Z \leq 6\left(\mathrm{He} \mathrm{Li}^{+}, \mathrm{Be}^{2+}, \mathrm{B}^{3+}, \mathrm{C}^{4+}, \mathrm{O}^{6+}\right)$.

[553] K. Maeda, K. Ueda, K. Ito and T. Namioka. Absorption cross section of krypton in the photoionization threshold region. PS, 41:464-7, 1990. Exp-Keys : 0U - Species : Kr.

[554] V.K. Mago, B. Lal, A.K. Ray, R. Kapoor, S.D. Sharma and P.R.K. Rao. Two-colour three-step photoionization of uranium. JPB, 20:6021-30, 1987. Exp-Keys : FP - Species : U.

[555] V.K. Mago, A.K. Ray, B. Lal and P.R.K. Rao. Study of high-lying odd levels in U I by two-colour photoionisation. JPB, 21:955-61, 1988. Exp-Keys : FP - Species : U. 
[556] K.S. Mann, N. Singh, R. Mittal, K.L. Allawadhi and B.S. Sood. M-shell X-ray production cross section in Au, $\mathrm{Pb}$, Th and U by 6-12 keV photons. JPB, 23:2497-504, 1990. Exp - Keys : IN - Species : Au, Pb, Th, U.

[557] S. Mannervik, H. Cederquist and E. Trabert. On the EUV spectrum of Li I. PS, 34:143-5, 1986. Exp - Keys : IN - Species : Li.

[558] K.G. Manohar, P.N. Bajaj, B.M. Suri, R. Talukdar, K. Dasgupta, P.K. Chakraborti and P.R.K. Rao. Observation of autoionization resonances in uranium by step-wise laser photoionization. APB, B48:525-30, 1989. Exp-Keys : AI FP - Species: U.

[559] S.T. Manson. Photoionization of the excited 3p state of nitrogen. PRA, 38:126-7, 1988. Theory - Keys : 00 Species : N.

[560] S.T. Manson. Photoionization cross sections of positive ions. PRA, 38:506-7, 1988. Theory - Keys : OU Species: $\mathrm{K}^{+}, \mathrm{K}$.

[561] S.T. Manson. Photoionisation of positive atomic ions : a review of our present understanding. AIPCP, 205:189200, 1989. Th. review - Keys : 0U - General.

[562] S.T. Manson. Photoionisation cross sections : present status and future needs. NLMPR, A280:173-9, 1989. Th. review - Keys : OU - General.

[563] N.B. Mansour, C.J. Edge and D.J. Larson. Laser photodetachment spectroscopy of Se and S$^{-}$. NIMPR, B31:313-16, 1988. Exp-Keys : FP - Species : $\mathrm{S}^{-}, \mathrm{Se}^{-}$.

[564] G. Marr. Some experimental work on photoionization of atoms in Europe. ADW2, p. 14-19, 1989. Exp. review - Keys : oU - General.

[565] F. Martin, A. Riera, M. Yanez and H. Bachau. Comparison of the conventional and pseudopotential Feshbach methods : $\mathrm{N}^{5+}\left(3 l 3 l^{\prime}\right){ }^{1} \mathrm{~S}^{\mathrm{e}}$ and ${ }^{1,3} \mathrm{P}^{\circ}$. JPB, 21:2261-72, 1988. Theory - Keys : AI - Species : $\mathrm{N}^{5+}$.

[566] F. Martin, I. Sanchez and H. Bachau. Coupled continuum perturbative Feshbach approach to calculate total and partial widths : ${ }^{1,3} \mathrm{~S}(3 \ln l)$ resonant states of $\mathrm{He}, \mathrm{C}^{4+}, \mathrm{N}^{5+}$ and $\mathrm{O}^{6+}$. PRA, 40:4245-55, 1989. Theory Keys : AI - Species : $\mathrm{He}, \mathrm{C}^{4+}, \mathrm{N}^{5+}, \mathrm{O}^{6+}$.

[567] N.L.S. Martin. Photoelectron angular distributions from zinc, cadmium and mercury. NIMPR, B40-41:228-31, 1989. Theory - Keys : oU - Species : Zn, Cd, Hg.

[568] M.C. Martins, M.I. Marques, F. Parente and J.G. Ferreira. Some K X-ray relative transition probabilities for $Z=47,49,52,55$ and 56. JPB, 22:3167-74, 1989. Exp-Keys : IN - Species : Ag, In, Te, Cs, Ba.

[569] N. Maskil and M. Deutsch. A high resolution study of the Cu K $\alpha$ satellite spectrum. JPCo, 48-C9:641-3, 1987. Exp - Keys : IN - Species : Cu.

[570] N. Maskil and M. Deutsch. X-ray $K \alpha$ satellites of copper. PRA, 38:3467-72, 1988. Exp \& theory - Keys : IN - Species : Cu.

[571] V.I. Matveev. Theory of two- and three-electron autoionizing states. SPJETP, 62:233-7, 1986. Theory - Keys : IN AI - Species : He seq, Li seq.

[572] M. Mazzoni and Y.N. Yoshi. Inner 4d-subshell photoabsorption spectrum of Sb I. JPB, 22:2485-92, 1989. Exp - Keys : IN - Species : Sb.

[573] M.P. McCann, C.H. Chen and M.G. Payne. Two-photon (vacuum ultraviolet + visible) spectroscopy of argon, krypton, xenon and molecular hydrogen. JCP, 89:5429-41, 1988. Exp - Keys : FP - Species : Ar, Kr, Xe.

[574] B.H. McQuaide, M.S. Banna, P. Gérard and M.O. Krause. Photoelectron spectrometry of mercury in the 17-40 photon energy range. PRA, 35:1603-6, 1987. Exp-Keys : OU - Species : Hg.

[575] B.H. McQuaide, M.D. Hawkins and M.S. Banna. Shake up in the core ionization of atomic zinc and cadmium. CPL, 141:306-8, 1987. Exp-Keys : IN - Species : Zn, Cd.

[576] B.H. McQuaide, M.D. Hawkins and M.S. Banna. Atomic cesium 3d core photoelectron spectrum. PRA, 36:4498500, 1987. Exp - Keys : IN - Species : Cs. 
[577] L.J. Medhurst, A. Schach von Wittenau, R.D. Van Zee, S. Zhang, S.H. Liu, D.A. Shirley and D.W. Lindle. Threshold photoelectron spectrum of the argon 3s sattelites. JESRP, 52:671-5, 1990. Exp - Keys : oU Species : Ar.

[578] J.D. Meiss. Comment on 'Microwave ionization of $\mathrm{H}$ atoms : breakdown of classical dynamics for high frequencies'. PRL, 62:1576, 1989. Exp - Keys : MP - Species : H.

[579] C. Mendoza and C.J. Zeippen. Photoionisation cross sections of the ground states of neutral Si, P and S. JPB, 21:259-66, 1988. Theory - Keys : OU - Species : Si, P, S.

[580] T. Mercouris and C.A. Nicolaides. Solution of the complex eigenvalue Schrödinger equation for the Rydberg series of inner hole excited autoionizing states. The Be $2 s^{2} s^{2} n p(n=2-5){ }^{3} \mathrm{P}^{\circ}$ and ${ }^{1} \mathrm{P}^{\circ}$ series. ZPD, 5:1-7, 1987 . Theory - Keys : AI - Species : Be.

[581] M. Meyer, B. Muller, A. Nunnemann, T. Prescher, E. von Raven, M. Richter, M. Schmidt, B. Sonntag and P. Zimmermann. Photoelectron spectroscopy of oriented and aligned alkali atoms. JPCo, 48-C9:551-4, 1987. Exp - Keys : OU - Species : Na.

[582] M. Meyer, B. Muller, A. Nunnemann, T. Prescher, E. von Raven, M. Richter, M. Schmidt, B. Sonntag and P. Zimmermann. Resonant vacuum-ultraviolet photoelectron spectra of aligned Li atoms. PRL, 59:2963-6, 1987. Exp - Keys : OU - Species : Li.

[583] M. Meyer, M. Pahler, Th. Prescher, E. von Raven, M. Richter, B. Sonntag, S. Baier, W. Fiedler, B.R. Muller, M. Schulze and P. Zimmermann. Photoelectron spectroscopy of laser-excited aligned free atoms. PS, T31:28-31, 1990. Exp - Keys : OU - General.

[584] M. Meyer, T. Prescher, E. von Raven, M. Richter, B. Sonntag, B.R. Muller, W. Fiedler and P. Zimmermann. Photoelectron spectroscopy of laser excited Ca atoms. JPCo, 48-C9:547-50, 1987. Exp-Keys : 0U - Species : $\mathrm{Ca}$.

[585] M. Meyer, T. Prescher, E. von Raven, M. Richter, E. Schmidt, B. Sonntag and H.E. Wetzel. Decay channels of core excitation resonances in atomic lanthanides and $3 \mathrm{~d}$ transition metals. NATO2, p. 251-5, 1987. Exp. review - Keys : IN - Species : Sc, Ti, V, Cr, Mn, Fe, Co, Ni, Cu, Zn, La.

[586] M. Meyer, E. von Raven, M. Richter, B. Sonntag, R.D. Cowan and J.E. Hansen. Autoionization of the Ca 2p 3 d core resonances : breakdown of the spectator model. PRA, 39:4319-22, 1989. Exp \& theory - Keys : IN AI Species : Ca.

[587] G. Miecznik, K.A. Berrington, P.G. Burke and A. Hibbert. Photoionisation of the ground state of singly ionized calcium. JPB, 23:3305-14, 1990. Theory - Keys : OU - Species : $\mathrm{Ca}^{+}$.

[588] T. Millack. $T$-matrix and $K$-matrix Floquet theory for atoms in strong laser fields. JPB, 23:1693-710, 1990. Theory - Keys : MP - General.

[589] T. Millack, B. Piraux and P.L. Knight. Non adiabatic transitions between degenerate opposite-parity states of hydrogen in an intense field. PRA, 41:5127-31, 1990. Theory - Keys : MP - Species : H.

[590] J.C. Miller. Two-photon resonant multiphoton ionization and stimulated emission in krypton and xenon. PRA, 40:6969-76, 1989. Exp - Keys : FP - Species : Kr, Xe.

[591] J.E. Miraglia and J. Macek. Post-collisional Stark mixing in Auger processes. PRA, 42:3971-83, 1990. Theory -Keys : IN - Species : He.

[592] M.N. Mirakhmedov and E.S. Parilis. Energy of atoms with several vacancies in inner shells. JPB, 21:775-93, 1988. Theory - Keys : IN AI - Species : Kr.

[593] M.N. Mirakhmedov and E.S. Parilis. Auger and X-ray cascades following inner-shell ionisation. JPB, 21:795804, 1988. Theory - Keys : IN - Species : Xe.

[594] V.I. Mishin and V.N. Fedoseev. Laser resonance photoionization spectroscopy of excited and autoionized states of atoms of rare-earth elements. 2. Gadolinium. OS, 63:420-2, 1988. Exp-Keys : FP OU AI - Species : Gd.

[595] M.H. Mittleman. Adiabatic theory of nonresonant multiphoton ionization. PRA, 40:463-6, 1989. Theory Keys : MP - General.

[596] M.H. Mittleman. Atoms in intense radiation fields. AIPCP, 205:184-8, 1989. Th. review - Keys : MP - General. 
[597] R. Moccia and P. Spizzo. An $L^{2}$ calculation of the ${ }^{1,3} \mathrm{P}^{\circ}$ resonances of atomic helium. JPB, 20:1423-31, 1987. Theory-Keys : AI - Species : He.

[598] R. Moccia and P. Spizzo. Atomic magnesium : I. Discrete and continuous energy spectrum below the 3p ionisation threshold. A valence-shell $L^{2}$ Cl calculation. JPB, 21:1121-32, 1988. Theory - Keys : oU - Species : $\mathrm{Mg}$.

[599] R. Moccia and P. Spizzo. Atomic magnesium : II. One-photon transition probabilities and ionisation cross sections. A valence-shell $L^{2}$ CI calculation. JPB, 21:1133-43, 1988. Theory - Keys : OU - Species : Mg.

[600] R. Moccia and P. Spizzo. Atomic magnesium : III. Two-photon transition probabilities and ionisation cross sections : A valence-shell $L^{2} \mathrm{CI}$ calculation. JPB, 21:1145-54, 1988. Theory - Keys : FP - Species : Mg.

[601] R. Moccia and P. Spizzo. Atomic magnesium : discrete and continuum spectrum properties from a valence-shell $L^{2}$ configuration-interaction calculation with polarization potential. PRA, 39:3855-60, 1989. Theory - Keys : oU - Species : $\mathbf{M g}$.

[602] R. Moccia and P. Spizzo. Lithium anion photodetachment up to the $3 \mathrm{~s}$ threshold: a $K$-matrix $L^{2}$ basis calculation. JPB, 23:3557-67, 1990. Theory-Keys : OU - Species : $\mathrm{Li}^{-}$.

6003] P. Monchicourt, P. Pradel, D. Dubreuil and J.J. Locagne. Laser assisted ionization as the only process consistent with experiment. PRA, 40:1147-9, 1989. Exp-Keys : MP - General.

[604] N. Morita and T. Suzuki. Spectroscopic observation of doubly excited 8sns and 7dns states Ca with multistep laser excitation. JPB, 21:L439-44, 1988. Exp-Keys : AI - Species : Ca.

[605] J.P. Mosnier, J. Brilly and E.T. Kennedy. Low lying odd parity autoionising states in the 2p-subshell absorption spectrum of Al III and Si IV. JPCo, 48-C9:219-22, 1987. Theory-Keys : AI - Species : $\mathrm{Al}^{2+}, \mathrm{Si}^{3+}$.

[606] A.M. Movsesyan and M.V. Fedorov. Interference phenomena in photoionization-like processes in a group of coherently populated Rydberg levels. SPJETP, 67:462-9, 1988. Theory - Keys : OU - General.

[607] A.M. Movsesyan and M.V. Fedorov. Interference effects in the photoionisation of Rydberg atoms in strong electromagnetic fields. SPJETP, 68:27-33, 1989. Theory - Keys : MP - General.

[608] A.Z. Msezane, F. Nyandeh and J. Niles. Coupling effects in photoionization of ground state Na : $R-$ matrix calculations. JPB, 23:L691-95, 1990. Theory - Keys : OU - Species : Na.

[609] X. Mu. Multiphoton absorption probabilities in strong laser fields with application to $\mathrm{H}^{-}$. PRA, 42:2944-8, 1990. Theory - Keys : MP - Species : $\mathrm{H}^{-}$.

[610] X. Mu and B. Crasemann. Two-photon transitions in atomic inner shells : a relativistic self-consistent-field calculation with applications to $\mathrm{Mo}, \mathrm{Ag}$ and Xe. PRA, 38:4585-96, 1988. Theory - Keys : FP IN - Species : Mo, Ag, Xe.

[611] X. Mu, J. Ruscheinski and B. Crasemann. Multiphoton detachment rate of $\mathrm{H}^{-}$in strong laser fields : effect of final state interaction. PRA, 42:2949-52, 1990. Theory - Keys : MP - Species : $\mathrm{H}^{-}$.

[612] T. Mukoyama. Electron shakeoff accompanying photoionization. BICRKU, 64:1-11, 1986. Theory - Keys : IN - General.

[613] T. Mukoyama and K. Taniguchi. Atomic excitation as the result of inner-shell vacancy production. PRA, 36:693-8, 1987. Theory - Keys : IN - Species : All $2 \leq Z \leq 36$.

[614] T. Mukoyama, T. Tonuma, A. Yagishita, H. Shibata, T. Koizumi, T. Matsuo, K. Shima and H. Tawara. Charge distribution of Xe ions as a result of multiple photoionisation of Xe atoms between 4.1 and $8.0 \mathrm{keV}$. JPB, 20:4453, 1987. Exp \& theory - Keys : MI - Species : Xe.

[615] H.G. Muller. The phase problem in multiphoton ionization. CAMP, 24:355-64, 1990. Review - Keys : MP General.

616] H.G. Muller, P.H. Bucksbaum, D.W. Schumacher and Zavriyev A. Above-threshold ionisation with a two-colour laser field. JPB, 23:2761-9, 1990. Exp-Keys : MP - Species : He, Ne.

[617] H.G. Muller and A. Tip. Atoms in multi-mode radiation fields. JPB, 19:903-17, 1986. Theory - Keys : MP General. 
[618] M. Muller, N. Böwering, F. Schäfers and U. Heinzmann. Spin-resolved photoionisation of lead above th $\mathrm{Pb}^{+}\left(6 \mathrm{~s}^{2} 6 \mathrm{p}\right){ }^{2} \mathrm{P}_{3 / 2}$ threshold. PS, 41:38-41, 1990. Exp - Keys : ou - Species : Pb.

[619] M. Muller, N. Böwering, F. Schäfers and U. Heinzmann. Photoelectron spin-polarisation spectroscopy in th $5 \mathrm{~s} 5 \mathrm{p}^{2}$ autoionisation region of indium. PS, 41:42-6, 1990. Exp-Keys : OU AI - Species : In.

[620] E. Murakami, T. Hayaishi, A. Yagishita and Y. Morioka. Multiple and partial photoionization cross sections is the Kr 3d ionization region. PS, 41:468-71, 1990. Exp - Keys : IN MI - Species : Kr.

[621] T. Nagata, Y. Itoh, T. Hayaishi, Y. Itikawa, T. Koisumi, T. Matsuo, Y. Sato, E. Shigemasa, A. Yagishita an M. Yoshino. Multiple photoionisation of $\mathrm{Cs}$ and Ba atoms due to creation of $4 \mathrm{~d}$ hole states. JPB, 22:3865-8C 1989. Exp-Keys : MI - Species : Cs, Ba.

[622] T. Nagata, M. Yoshino, T. Hayaishi, Y. Itikawa, Y. Itoh, T. Koizumi, T. Matsuo, Y. Sato, E. Shigemasa Y. Takizawa and A. Yagishita. 4d-shell photoionization of $\mathrm{Xe}, \mathrm{Cs}, \mathrm{Ba}, \mathrm{Sm}, \mathrm{Eu}$ and $\mathrm{Yb}$ atoms studied b: photoion-yield spectra. PS, 41:47-50, 1990. Exp-Keys : IN - Species : Xe, Cs, Ba, Sm, Eu, Yb.

[623] S.N. Nahar and S.T. Manson. Photoelectron angular distribution of the excited $2 \mathrm{p}^{2} 3 \mathrm{p}{ }^{2} \mathrm{~S}$ states of atomi nitrogen. PRA, 40:5017-19, 1989. Theory-Keys: DU - Species : N.

[624] S.N. Nahar and S.T. Manson. Photoionization of the 7d excited state of cesium. PRA, 40:6300-2, 1989. Theor: - Keys : OU - Species : Cs.

[625] L. Nahon, L. Duffy, P. Morin, F. Combet-Farnoux, J. Tremblay and M. Larzilliere. Relaxation of the 4d $\rightarrow$ 5] resonance in atomic iodine. PRA, 41:4879-88, 1990. Exp \& theory-Keys : IN - Specjes : I.

[626] G. Nasreen, P.C. Deshmukh and S.T. Manson. Photoionisation of $\mathrm{K}^{+}$ions. JPB, 21:L281-3, 1988. Theory . Keys : OU - Species : $\mathrm{K}^{+}$.

[627] G. Nasreen, S.T. Manson and P.C. Deshmukh. Photoionization of inner shells of the $\mathrm{Mg}$ and Ar isonuclea sequences. PRA, 40:6091-4, 1989. Theory-Keys : IN - Species : $\mathrm{Mg}^{8+}, \mathrm{Mg}^{2+}, \mathrm{Ar}^{8+}, \mathrm{Mg}, \mathrm{Ar}^{6+}, \mathrm{Ar}^{2}$.

[628] I. Nenner, P. Morin and P. Lablanquie. Double photoionization via inner shell excitation. CAMP, 22:51-65 1988. Exp. review - Keys : IN MI - General.

[629] C.A. Nicolaides, M. Chrysos and Y. Komninos. The geometry of the Wannier two-electron ionisation ladde and the corresponding spectrum. JPB, 21:L73-6, 1988. Theory - Keys : AI - Species : $\mathrm{H}^{-}, \mathrm{He} \mathrm{Li}^{+}, \mathrm{He}^{-}, \mathrm{Li}^{-}$

[630] C.A. Nicolaides and Y. Komninos. Energy dependence of the partial widths of the Wannier two-electrol ionization ladder states. JPB, 23:L571-3, 1990. Theory - Keys : AI - Species : $\mathrm{H}^{-}$.

[631] C.A. Nicolaides, N. Makri and Y. Komninos. Wavefunctions and autoionisation of doubly excited states is momentum space. JPB, 20:4963, 1987. Theory - Keys : AI - Species : He.

[632] C.A. Nicolaides and T. Mercouris. Multiphoton ionization of negative ions in the presence of a DC field Application to $\mathrm{Li}^{-}$. CPL, 159:45-50, 1989. Theory - Keys : SF MP - Species : $\mathrm{Li}^{-}$.

[633] C.A. Nicolaides, T. Mercouris and G. Aspromalis. Many-electron, many-photon theory of nonlinear polarisabil ities. JOSAB, 7:494-501, 1990. Theory - Keys : MP MI - Species : H, $\mathrm{Li}^{-}$.

634] C.A. Nicolaides, T. Mercouris and N.A. Piangos. Dynamic polarizability and hyperpolarizability of $\mathrm{H}^{-}$fo frequencies below as well as above the ionization threshold. JPB, 23:L669-76, 1990. Theory - Keys : OU Species : $\mathrm{H}^{-}$.

635] P. Nicolosi, E. Janitti and G. Tondello. A review on experimental studies of the photoabsorption spectra of lov $Z$ ions. JPCo, this issue, 1990. Exp. review - Keys : OU - Species : $\mathrm{Be}^{3+}, \mathrm{Be}^{2+}, \mathrm{B}^{3+}, \mathrm{C}^{4+}, \mathrm{Be}^{+}, \mathrm{B}^{2+}, \mathrm{C}^{3+}, \mathrm{Be}^{2}$ $\mathrm{B}^{+}, \mathrm{C}^{2+}$.

[636] J. Nilsen. Resonant photoionization for sodiumlike selenium. PRA, 38:2167-70, 1988. Exp - Keys : oU Species: $\mathrm{Se}^{23+}$.

[637] G.P. Nizhnikova, S.V. Vlasov, E. Remeta and O.V. Farberovich. Calculation of energies of autoionization state by the method of the local-density functional. OS, 62:696-7, 1987. Theory - Keys : AI - Species : He, Be, Mg Ca. 
[638] L.D. Noordam, J.W.J. Verschuur, P. Agostini, H.G. Muller, P. Breger, A. Migus, and H.B. van Linden van der Heuvell. Absence of resonances in femtosecond photoionisation spectra. JPB, 22:L57-63, 1989. Exp - Keys : OU - Species : Xe.

[639] M. Ohno. Many-body theory of multiple core hole. JPCo, 48-C9:505-8, 1987. Theory - Keys : IN - GeneraJ.

[640] M. Ohno. Many-body calculation of the 3p-3d X-ray emission spectrum of krypton. JESRP, 46:165-71, 1988. Exp - Keys : IN - Species : Kr.

[641] M. Ohno. Many-body calculations of the X-YY Auger energies of the atomic elements Ar, Kr, Sr and Xe. JESRP, 48:125-35, 1988. Theory - Keys : IN - Species : Ar, Kr, Sr, Xe.

[642] M. Ohno. Green's function calculation of the Auger energy. PRA, 38:3473-8, 1988. Theory - Keys : IN Species : Xe.

[643] M. Ohno and R.E. LaVilla. Anomalous $L \gamma_{2,3}$ X-ray emission spectrum of Xe. PRA, 38:3479-83, 1988. Exp \& theory - Keys : IN - Species : Xe.

[644] K. Okuyama, J.H.D. Eland and K. Kimura. Decay of the 4d hole states of Xe studied by photoelectronphotoelectron coincidence spectroscopy. PRA, 41:4903-5, 1990. Exp-Keys : IN - Species : Xe.

[645] M.G. Opendak. Auger cascades in atoms and ions of astrophysically important elements. ASI, 165:9-25, 1990. Th. review - Keys : IN - Species : C, N, O, Ne, Mg, Al, Si, S, Ar, Ca, Fe and their ions.

[646] A.E. Orel and T.N. Rescigno. An algebraic variational approach to the calculation of total cross sections for one- and two-photon ionization. CPL, 146:434-8, 1988. Theory - Keys : FP OU - Species : H.

[647] V.N. Ostrovsky and D.A. Telnov. Adiabatic theory of multiphoton ionisation. I. Ionisation of an isolated level. JPB, 20:2397-419, 1987. Theory - Keys : MP - General.

[648] V.N. Ostrovsky and D.A. Telnov. Adiabatic theory of multiphoton ionisation. II. Resonant phenomena. JPB, 20:2421-36, 1987. Theory - Keys : MP - General.

[649] V.D. Ovsyannikov. Orientation and alignment of ions during photoionization of atoms. OS, 63:579-82, 1988. Theory - Keys : FP - General.

[650] D.H. Oza. Autoionisation states $\left(3 / 3 l^{\prime}\right)$ of the helium-like nitrogen ion. JPB, 20:L13-17, 1987. Theory - Keys : AI - Species : $\mathrm{N}^{5+}$.

[651] D.H. Oza, P. Benoit-Cattin, A. Bordenave-Montesquieu, M. Boudjema and A. Gleizes. Autoionisation of $\mathrm{N}^{5+}\left(3 / n^{\prime} l^{\prime}\right)$ for $n^{\prime}=3-10$ : experiment and theory. JPB, 21:L131-7, 1988. Exp \& theory - Keys : AI - Species : $\mathrm{N}^{5+}$.

[652] C. Pan, B. Gao and A.F. Starace. Two-photon ionization of the Ar atom and detachment of the F- ion. PRA, 41:6271-86, 1990. Theory - Keys : FP - Species : F-, Ar.

[653] L. Pan. The Volkov-like Coulomb continuum. JMO, 36:877-89, 1989. Theory - Keys : MP - General.

[654] L. Pan, B. Sundaram and L. Armstrong Jr. Dressed state perturbation theory for multiphoton ionization of atoms. JOSAB, 4:754-9, 1987. Th. review - Keys : FP - Species : H.

655] S.M. Park and G.J. Diebold. Selective ionization of $\mathrm{Ba}$ and $\mathrm{Sr}$ isotopes based on a two-photon interference effect. PRA, 42:417-23, 1990. Exp - Keys : FP - Species : Sr, Ba.

[656] J. Parker and C.R. Stroud Jr. Generalization of the Keldysh theory of above-threshold ionization for the case of femtosecond pulses. PRA, 40:5651-8, 1989. Theory - Keys : MP - Species : H.

[657] S.D. Parker and C.W. McCurdy. Propagation of wave packets using the complex basis function method. CPL, 156:483-8, 1989. Theory - Keys : MP - Species : H.

[658] K. Parthasaradhi, A. Esposito, S. Mobilio and M. Pelliccioni. Photon cross sections near the X-ray absorption edges of Ti, Ni, Pt and Au. PRA, 38:1608-11, 1988. Exp-Keys : IN-Species : Ti, Ni, Pt, Au.

[659] G. Peach, H.E. Saraph and M.J. Seaton. Atomic data for opacity calculations. IX. The lithium isoelectronic sequence. JPB, 21:3669-83, 1988. Theory-Keys : oU -Species : Li seq with $3 \leq Z \leq 10\left(\mathrm{Li}_{1} \mathrm{Be}^{+}, \mathrm{B}^{2+}, \mathrm{C}^{3+}\right.$, $\left.\mathrm{N}^{4+}, \mathrm{O}^{5+}, \mathrm{F}^{6+}, \mathrm{Ne}^{7+}\right)$. 
[660] B. Peart, I.C. Lyon and K. Dolder. Measurements of absolute photoionisation cross sections of $\mathrm{Ga}^{+}$and $\mathrm{Zn}^{+}$ ions. JPB, 20:5403-10, 1987. Exp-Keys: OU - Species : $\mathrm{Zn}^{+}, \mathrm{Ga}^{+}$.

[661] D.J. Pegg. Photodetachment collisions. AIPCP, 205:233-240, 1990. Exp-Keys : OU - Species : He ${ }^{-}, \mathrm{B}^{-}$.

[662] D.J. I'egg, J.S. Thompson, R.N. Compton and G.D. Alton. Energy and angle resolved photoelectron spectroscopy of negative ions. NIMPR, B40-41:221-4, 1989. Exp-Keys : OU - Species : $\mathrm{He}^{-}, \mathrm{Ca}^{-}$.

[663] D.J. Pegg, J.S. Thompson, J. Dellwo, R.N. Compton and G.D. Alton. Partial cross sections for the photodetachment of metastable $\mathrm{He}^{-}$. PRL, 64:278-81, 1990. Exp-Keys : OU - Species : $\mathrm{He}^{-}$.

[664] M. Pellarin, J.L. Vialle, M. Carre, J. Lerme and M. Aymar. Even parity series of argon Rydberg states studied by fast-beam collinear laser spectroscopy. JPB, 21:3833-49, 1988. Exp \& theory - Keys : FP - Species : Ar.

[665] R.C.C. Perera, M.C. Hettrick and D.W. Lindle. High resolution krypton $M_{4,5}$ X-ray emission spectra. JPCo, 48-C9:645-8, 1987. Exp-Keys : IN - Species : Kr.

[666] M.D. Perry and O.L. Landen. Resonantly enhanced multiphoton ionization of krypton and xenon with intense ultraviolet laser radiation. PRA, 38:2815-29, 1988. Exp-Keys : MP - Species : Kr, Xe.

[667] M.D. Perry, A. Szoke, O.I. Landen and Carnpbell E.M. Nonresonant multiphoton ionization of noble gases : theory and experiment. PRL, 60:1270-3, 1988. Exp \& theory - Keys : MP - Species : Ar, Kr, Xe .

[668] G. Petite, P. Agostini and H.G. Muller. Intensity dependence of non-perturbative above-threshold ionisation spectra : experimental study. JPB, 21:4097-105, 1988. Exp-Keys : MP - Species : Xe.

[669] G. Petite and P. Agostini. Electron energy spectra in high intensity multiphoton ionisation. MP, p. 12-24, 1988. Exp. review - Keys : MP - Species : Sr, Xe.

[670] G. Petite, P. Agostini and F. Yergeau. Intensity, pulse width and polarization dependence of above-thresholdionization electron spectra. JOSAB, 4:765-9, 1987. Exp. review - Keys : MP - Species : Xe.

[671] D. Petrini and M.A. Delgado. Photoionisation of some $C^{2+}$ excited terms. RBF, 18:233-42, 1988. Theory Keys : OU - Species : $\mathrm{C}^{2+}$.

[672] D. Petrini and E. Segre. Theoretical L-Auger line intensities and widths for some $2 \mathrm{~s}^{2} \mathrm{p}^{6} \mathrm{n}^{\prime} n^{\prime} l^{\prime} \mathrm{Na}$ terms. JPB, 20:3697, 1987. Theory - Keys : IN AI - Species : Na.

[673] M.S. Pindzola and C. Bottcher. Strong-ficld laser ionization of model atoms. JOSAB, 4:752-3, 1987. Theory Keys : MP - General.

[674] M.S. Pindzola, G.J. Bottrell and C. Bottcher. Strong-field laser ionization of alkali atoms using two-dimensional cylindrical and three-dimensional Cartesian time-dependent Hartree-Fock theory. JOSAB, 7:659-63, 1990. Theory - Keys : MP - Species : H, Li.

[675] B. Piraux and P.L. Knight. Role of counterrotating wave dynamics in near threshold photoionization of atomic hydrogen. PRA, 40:712-26, 1989. Theory - Keys : MP - Species : H.

[676] M. Poirier. Autoionization of Rydberg states with large angular momentum : application to alkaline-earth atoms. PRA, 38:3484-97, 1988. Theory - Keys : AI - Species : Sr, Ba.

[677 M. Poirier. Asymptotic expressions for bound-free Coulomb matrix elements ; application to autoionisation processes of alkaline-earth atoms. JPB, 22:1537-53, 1989. Theory - Keys : AI - Species : Ba.

[678] M. Polasik. Theoretical multiconfiguration Dirac-Fock method study on the X-ray spectra of multiply ionized heavy atoms : the structure of the $K \alpha L^{0} M^{\gamma}$ lines. PRA, 40:4361-8, 1989. Theory - Keys : IN - Species : Mo, $\mathrm{Pd}$, Ho and their ions.

[679] M. Pont, R. Shakeshaft and Potvliege R.M. Low-frequency theory of multiphoton ionization. PRA, 42:6969-72, 1990. Theory - Keys : MP - General.

[680] R.M. Potvliege and R. Shakeshaft. Movement and interplay of the bound state, resonance and shadow poles of the scattering amplitude in multiphoton processes. PRA, 38:6190-203, 1989. Theory - Keys : MP - General.

[681] R.M. Potvliege and R. Shakeshaft. Atomic processes in intense laser fields. SPIG88, p. 111-35, 1989. Th. review - Keys : MP - General. 
[682] R.M. Potvliege. Multiphoton processes in an intense laser field. II. Partial rates and angular distributions for ionization of atomic hydrogen at $532 \mathrm{~nm}$. PRA, 41:1609-19, 1990. Theory - Keys : MP - Species : H.

[683] R.M. Potvliege and R. Shakeshaft. Nonperturbative calculation of partial differential rates for multiphoton ionization of a hydrogen atom in a strong laser field. PRA, 38:1098-1100, 1988. Theory - Keys : MP - Species : H.

[684] R.M. Potvliege and R. Shakeshaft. Time-independent theory of multiphoton ionization of an atom by an intense field. PRA, 38:4597-621, 1988. Theory - Keys : MP - Species : H.

[685] R.M. Potvliege and R. Shakeshaft. High-order above-threshold ionization of hydrogen in pertubation theory. PRA, 39:1545-8, 1989. Theory - Keys : MP - Species : H

[686] R.M. Potvliege and R. Shakeshaft. Multiphoton processes in an intense laser field : harmonic generation and total ionization rates for atomic hydrogen. PRA, 40:3061-79, 1989. Theory - Keys : MP - Species : H.

[687] A.K. Pradhan. Atomic data for opacity calculations: carbon and silicon isoelectronic sequences. JPCo, this issue, 1990. Theory - Keys : OU - Species : C seq with $6 \leq Z \leq 26$, Si seq with $14 \leq Z \leq 26$.

[688] T. Prescher, E. von Raven, M. Richter and B. Sonntag. Photoelectron spectroscopy of atomic Ca in the 2pexcitation range. JPCo, 48-C9:543-6, 1987. Exp-Keys : IN - Species : Ca.

[689] S.D. Price and J.H.D. Eland. Single-photon double ionisation of xenon. JPB, 22:L153, 1989. Exp - Keys : MI - Species : Xe.

[690] S.D. Price and J.H.D. Eland. Single photon double ionisation of mercury. JPB, 23:2269-76, 1990. Exp - Keys : MI - Species: Hg.

[691] L. Pruvost, A. Bolovinos, P. Camus, J.M. Lecomte and P. Pillet. UV laser spectroscopy of 7pnd doubly excited autoionising states of barium. JPB, 23:L95-102, 1990. Exp - Keys : AI - Species : Ba.

[692] J.Z. Qiu, Z. Zhang and Wang. G. Resonances of photoionisation of $\mathrm{Ca}$ and $\mathrm{Sr}$ atoms in an electric field. APS, 38:1172-6, 1989. Exp-Keys : SF AI - Species : Ca, Sr.

[693] A. Raczynski and J. Zaremba. Threshold effects in strong-field photoionisation of atoms. JPB, 19:3895-907, 1986. Theory - Keys : MP - General.

[694] A. Raczynski and J. Zaremba. Near-threshold photoelectron spectrum in resonant two-photon ionization of atoms. PRA, 36:5079, 1987. Theory - Keys : FP - General.

[695] A. Raczynski and J. Zaremba. Near-threshold photoionization and photodetachment by an exponentially decaying pulse. PRA, 38:5115-20, 1988. Theory - Keys : DU - General.

[696] A. Raczynski and J. Zaremba. Threshold effects in photodetachment to a structured continuum. PRA, 40:18437, 1989. Theory - Keys : oU - General.

[697] A. Raczynski and J. Zaremba. Dynamics of a near-threshold photodetachment to a structured continuum. JPB, 23:3105-12, 1990. Theory - Keys : OU - General.

[698] V. Radojevic, H.P. Kelly and Z. Altun. Photoionization of the 4d shell of barium. PS, 41:823-6, 1990. Theory - Keys : IN - Species : Ba.

[699] V. Radojevic, M. Kutzner and H.P. Kelly. Photoionization of the barium 4d subshell including relativistic and relaxation effects. PRA, 40:727-34, 1989. Theory - Keys : IN - Species : Ba.

[700] V. Radojevic and J.D. Talman. Calculation of Beutler-Fano autoionising resonances in neon : $1 s^{2} 2 s^{2} 2 p^{5}\left({ }^{2} P_{1 / 2}\right) n s$ and $n d_{3 / 2}$ series. JPB, 23:2241-6, 1990. Theory - Keys : AI - Species: Ne.

[701] G. Ramesh Babu, V. Gopalakrishna, M. L. N. Raju, K. Parthasaradhi, V. Radha Krishna Murty, M.V.R. Murti and K.S. Rao. K $\alpha$ X-ray satellites of $\mathrm{P}, \mathrm{S}, \mathrm{Cl}, \mathrm{K}$ and $\mathrm{Ca}$ excited by photons. PRA, 36:386-9, 1987. Exp Keys : IN - Species : P, S, Cl, K, Ca.

[702] S. Rashid. Approximate solution of the hydrogenlike atoms in intense laser radiation. PRA, 40:4242-4, 1989. Theory - Keys : MP - Species : H.

[703] A.R.P. Rau. Implications of electron correlations in multiphoton excitation. JOSAB, 4:784-7, 1987. Review Keys : FP - Species : Ca. 
[704] A.R.P. Rau and H.Y. Wong. Electric-field-induced modulations in photodetachment. PRA, 37:632-5, 1988. Theory-Keys : $\mathrm{SF}$ - Species : $\mathrm{H}^{-}$.

[705] S. Ravi and G.S. Agarwal. Field dependence of the autoionization from two close-lying states. PRA, 38:6437-8, 1988. Theory - Keys : SF AI - General.

[706] A.K. Ray, V.K. Mago, B. Lal and P.R.K. Rao. New odd-parity Rydberg and autoionization levels in uranium I. JOSAB, 7:145-51, 1990. Exp-Keys : AI - Species : U.

[707] V.C. Reed and K. Burnett. Ionization of atoms in intense laser pulses using the Kramers-Henneberger transformation. PRA, 42:3152-55, 1990. Theory - Keys : MP - General.

[708] W.P. Reinhardt and D.R. Kerner. Wavepacket dynamics as a tool for calculation of averaged photoionisation cross sections. AIPCP, 205:458-65, 1990. Theory-Keys : OU - Species : H, He, Li.

$\{709\}$ H.R. Reiss. Electron spectrum in intense-field photoionization. JOSAB, 4:726-30, 1987. Th. review - Keys : MP - General.

[710] H.R. Reiss. Spectrum of atomic electron ionised by an intense field. JPB, 20:L79-83, 1987. Theory - Keys : MP - Species : Xe.

[711] H.R. Reiss. Photoionization of arbitrary S states by extremely intense fields. APPA, A78:199-212, 1990. Theory - Keys : MP - General.

[712] H.R. Reiss. Relativistic strong-field photoionization. JOSAB, 7:574-86, 1990. Theory - Keys : MP - General.

[713] C.K. Rhodes. Physical processes at high field strengths. PS, T17:193-200, 1987. Th. review - Keys : MP Species : Ar, Xe.

[714] D. Richards, J.G. Leopold, P.M. Koch, E.J. Galvez, K.A.H. van Leeuwen, L. Moorman, B.E. Sauer and R.V. Jensen. Structure in low frequency microwave ionisation of excited hydrogen atoms. JPB, 22:1307-33, 1989. Exp \& theory - Keys : MF - Species : H.

[715] D. Richards and J.G. Leopold. Classical ghosts in quantal microwave ionization. AIPCP, 205:492-9, 1990. Theory - Keys : MP - Species : H.

[716] M. Richter, J.M. Bizau, D. Cubaynes, T. Menzel, F.J. Wuilleumier and B. Carre. 2s photoionisation in ground state and in laser-excited sodium atoms. EL, 12:35-40, 1990. Exp - Keys : IN - Species : Na.

[717] M. Richter, M. Meyer, M. Pahler, T. Prescher, E. von Raven, B. Sonntag and H.E. Wetzel. Experimental study of atomic $4 \mathrm{~d}$ giant resonances by photoabsorption and photoelectron spectroscopy : Ba, La and Ce. PRA, 39:5666-75, 1989. Exp - Keys : oU - Species : Ba, La, Ce.

[718] M. Richter, M. Meyer, M. Pahler, T. Prescher, E. von Raven, B. Sonntag and H.E. Wetzel. Experimental study of atomic $4 \mathrm{~d}$ giant resonances by photoabsorption and photoelectron spectroscopy : Sm, Eu and Gd. PRA, 40:7007-19, 1989. Exp - Keys : oU - Species : Sm, Eu, Gd.

[719] L. Roso-Franco and J.H. Eberly. Multiphoton above-threshold detachment computed for a model negative ion and compared with perturbation theory. JOSAB, 7:407-13, 1990. Theory - Keys : MP - General.

[720] H. Rottke and K.H. Welge. Photoionization of the hydrogen atom near the ionization limit in strong electric fields. PRA, 33:301-11, 1986. Exp-Keys : SF - Species : H.

[721] H. Rottke, B. Wolf, M. Brickwedde, D. Feldmann and K.H. Welge. Multiphoton ionization of atomic hydrogen in intense subpicosecond laser pulses. PRL, 64:404-7, 1990. Exp-Keys : MP - Species : H.

[722] B.F. Rozsnai. Inner-shell photoionisation of barium, xenon and krypton. PRA, 42:286-90, 1990. Theory Keys : IN - Species : $\mathrm{Kr}, \mathrm{Xe}, \mathrm{Ba}^{2+}, \mathrm{Ba}^{+}, \mathrm{Ba}$.

[723] B. Ruscic and J. Berkowitz. Autoionization in atomic chlorine : comparison of theories and experiment. PRA, 40:6716-18, 1989. Exp \& theory - Keys : OU AI - Species : Cl.

724] A. Russek and W. Mehlhorn. Post-collision interaction and the Auger line shape. JPB, 19:911-27, 1986. Theory - Keys : IN - Species : Ar.

[725] A.N. Ryabtsev. Autoionizing states in moderately charged ions. NIMPR, B31:196-205, 1988. Exp - Keys : AI - Species : K seq, Cu seq. 
[726] K. Rzazewski. Ionization rates at high laser intensity. APPA, A78:213-20, 1990. Theory - Keys : MP - General.

[727] K. Rzazewski, L. Wang and J.W. Haus. Predictions from a two-color above-threshold ionization model. JOSAB, 7:481-7, 1990. Theory - Keys : MP - Species : H.

[728] H.R. Sadeghpour and C.H. Greene. Anisotropy of excited $\mathrm{He}^{+}$formed in the photoionization of helium. PRA, 39:115-25, 1989. Theory - Keys : OU - Species : He.

[729] H.P. Saha. Multiconfiguration Hartree Fock calculation for the bound free photodetachment cross section of $\mathrm{H}^{-}$. PRA, 38:4546-51, 1988. Theory - Keys : OU - Species : $\mathrm{H}^{-}$.

[730] H.P. Saha. Threshold behavior of the M-shell photoionization of argon. PRA, 39:2456, 1989. Theory - Keys : DU - Species : Ar.

[731] H.P. Saha. Numerical multiconfiguration self-consistent-field studies at atomic photoionization cross sections : dynamical core-polarization effects in atomic potassium. PRA, 39:628-33, 1989. Theory - Keys : OU - Species : $\mathbf{K}$.

[732] H.P. Saha. Multiconfiguration Hartree-Fock calculation of the photoionization of the Cs 7d excited state. PRA, 41:174-80, 1990. Theory - Keys : OU - Species : Cs.

[733] H.P. Saha. Near-threshold $K$-shell photoionization of argon. PRA, 42:6507-11, 1990. Theory - Keys : IN Species : Ar.

[734] H.P. Saha and C.D. Caldwell. K-shell photoionization of beryllium. PRA, 40:7020-5, 1989. Theory - Keys : IN - Species : Be.

[735] H.P. Saha and R.N. Compton. Theoretical studies of the photophysics of $\mathrm{He}^{-}(1 \mathrm{~s} 1 \mathrm{~s} 2 \mathrm{p})^{4} \mathrm{P}^{\circ}$. PRL, 64:1510-13, 1990. Theory - Keys : OU - Species : $\mathrm{He}^{-}$.

[736] H.P. Saha, C. Froese-Fischer and P.W. Langhoff. Numerical multiconfiguration Hartree-Fock studies of atomic photoionization cross sections : dynamical core-polarization effects in atomic sodium. PRA, 38:1279-85, 1988. Theory - Keys : oU - Species : Na.

[737] H.P. Saha, M.S. Pindzola and R.N. Compton. Multiconfiguration Hartree-Fock calculation of the photoionization of the excited Na 4d state. PRA, 38:128-34, 1988. Theory - Keys : OU - Species : Na.

[738] K. Sakimoto, M. Terao and K.A. Berrington. Effects of radiative decay on the bound-continuum transitions of highly-charged atomic ions. PRA, 42:291-5, 1990. Theory-Keys : OU - Species : $\mathrm{Fe}^{24+}, \mathrm{Xe}^{52+}$.

[739] L. Salgueiro, M.L. Carvalho and F. Parente. Origin of the observed $L \beta_{2}$ satellite X-rays of tungsten not predicted by theory. JPCo, 48-C9:609-12, 1987. Exp-Keys : IN - Species : W.

[740] S. Salomonson, S.L. Carter and H.P. Kelly. Calculation of helium photoionization with excitation including angular distribution and resonance structure. PRA, 39:5111-16, 1989. Theory - Keys : OU - Species : He.

[741] D.H. Sampson, H.L. Zhang and A.K. Mohanty. Rate coefficients for populating excited levels of Ni-like ions by inner shell ionization of Cu-like ions. PRA, 38:4569-73, 1988. Theory - Keys : IN - Species : $\mathrm{Gd}^{35+}$.

[742] J.A.R. Samson and G.C. Angel. Test of the threshold law for triple photoionization in atomic oxygen and neon. PRL, 61:1584-6, 1988. Exp-Keys : MI - Species : O, Ne.

[743] J.A.R. Samson. Experimental work on photoionization in the USA. ADW2, p. 7-13, 1989. Exp. review - Keys : OU IN AI - Species: $\mathrm{Li}, \mathrm{Ne}, \mathrm{Cl}, \mathrm{Ar}, \mathrm{Br}, \mathrm{Kr}, \mathrm{Hg}, \mathrm{Pb}$.

[744] J.A.R. Samson. Proportionality of electron-impact ionization to double photoionization. PRL, 65:2861-4, 1990. Exp - Keys : MI - Species : N, O, Ne.

[745] J.A.R. Samson and G.C. Angel. Double-photoionization of $\mathrm{Ne}, \mathrm{O}$ and $\mathrm{N}$ from threshold to $280 \mathrm{eV}$. PRA, 42:5328-37, 1990. Exp - Keys : MI - Species : N, O, Ne.

[746] J.A.R. Samson, Y. Chung and E.M. Lee. Ar 3s, 3p satellite lines studied by fluorescence spectroscopy. PLA, 127A:171-4, 1988. Exp - Keys : OU - Species : Ar.

[747] J.A.R. Samson and L. Yin. Precision measurements of photoabsorption cross sections of Ar, Kr, Xe and selected molecules at 58.4, 73.6 and $74.4 \mathrm{~nm}$. JOSAB, 6:2326-33, 1989. Exp - Keys : OU - Species : Ar, Kr, Xe. 
[748] J.A.R. Samson, L. Yin, G.N. Haddad and G.C. Angel. Recent progress on the measurement of absolute atomic photoionization cross sections. JPCo, this issue, 1990. Exp. review - Keys : oU - Species : N, O, Ne, Cl, Ar, $\mathrm{Kr}, \mathrm{Xe}$.

[749] I. Sanchez and F. Martin. Photoionization of He-like systems below the $N=2$ threshold. JPB, 23:4263-74, 1990. Theory - Keys : OU - Species : $\mathrm{He}, \mathrm{Li}^{+}$.

[750] L. Sanders, S.D. Hanton and J.C. Weisshaar. Resonant two photon ionization of V atoms: $\mathrm{V}^{+}$electronic state distributions and $J$-resolved photoelectron angular distributions. JCP, 92:3485-97, 1990. Exp - Keys : FP Species : V.

[751] W. Sandner. Autoionizing Rydberg states. CAMP, 20:171-87, 1987. Review - Keys : AI SF - Species : Ba.

[752] W. Sandner, U. Eichmann, V. Lange and M. Volkel. Barium autoionising Rydberg series in the vicinity of a shape resonance. JPB, 19:51-71, 1986. Exp-Keys : DU AI - Species : Ba.

[753] W. Sandner and M. Volkel. Auger line shapes of free atoms. PRL, 62:885-8, 1989. Theory - Keys : IN Species : Ar.

[754] A. Sanpera and L. Roso-Franco. Short-time multiphoton detachment in a one-dimensional model with veryshort-range potential. PRA, 41:6515-18, 1990. Theory - Keys : MP - General.

[755] H.E. Saraph. A review of available data for photoionisation cross-sections. ADW2, p. 83-89, 1989. Th. review - Keys : oU -- General.

[756] H.E. Saraph. Atomic data for opacity calculations : The H, He and Li sequences, Fe VIII and Fe VII. JPCo, this issue, 1990. Theory - Keys : OU - Species : $\mathrm{H}$ seq, He seq, Li seq, $\mathrm{Fe}^{7+}, \mathrm{Fe}^{6+}$.

[757] R.P. Saxon, R.K. Nesbet and C.J. Noble. Photoionization of excited atomic oxygen : theory and calculations. PRA, 39:1156-62, 1989. Theory - Keys : OU - Species : O.

[758] F. Schäfers, C. Heckenkamp, G. Schonhense and U. Heinzmann. Energy dependence of the electron spin polarisation parameters for $\mathrm{Hg} 5 \mathrm{~d}$ photoionisation with circularly polarised light. JPB, 21:769-74, 1988. Exp - Keys : oU - Species : $\mathbf{H g}$.

[759] F. Schäfers, C. Heckenkamp, M. Muller, V. Radojevic and U. Heinzmann. Hg $5 \mathrm{~d}$ and $6 \mathrm{~s}$ : multichannel quantum defect analysis of experimental data. PRA, 42:2603-13, 1990. Exp-Keys : OU IN AI - Species : Hg.

[760] K.H. Schartner, B. Mobus, P. Lenz, H. Schmoranzer and M. Wildberger. Observation of resonances in the Ar-3s photoionization cross section. PRL, 61:2744-7, 1988. Exp-Keys : OU AI - Species : Ar.

[761] K.H. Schartner, P. Lenz, B. Mobus, H. Schmoranzer and M. Wildberger. Structures at the Xe 5s threshold studied by photon-induced fluoresence spectroscopy. JPB, 22:1573-81, 1989. Exp - Keys : 0U - Species : Xe.

[762] K.H. Schartner. Correlation effects in electron impact- and photon-induced two-electron transitions in rare gases. AIPCP, 205:215-223, 1990. Exp. review - Keys : OU AI - Species : Ar.

[763] K.H. Schartner, B. Magel, B. Mobus, H. Schmoranzer and M. Wildberger. The Ne 2s photoionisation cross section between threshold and $51 \mathrm{eV}$. JPB, 23:L527-32, 1990. Exp - Keys : OU - Species : Ne.

[764] K.F. Scheibner and A.U. Hazi. Photodetachment cross section of $\mathrm{Cu}^{-}$. PRA, 38:539-42, 1988. Theory - Keys : oU - Species : $\mathrm{Cu}^{-}$.

[765] M. Schmidt and P. Zimmermann. The giant resonances in the $3 \mathrm{~d}$ transition elements : single and double photoionization in the region of the 3p excitation. NATO2, p. 247-9, 1987. Exp. review - Keys: IN MI Species : $\mathrm{Sc}, \mathrm{Ti}, \mathrm{Cr}, \mathrm{Mn}, \mathrm{Fe}, \mathrm{Co}, \mathrm{Ni}, \mathrm{Cu}, \mathrm{Zn}$.

[766] V. Schmidt. PCI effects in inner-shell photoionization. JPCo, 48-C9:401-14, 1987. Exp. review - Keys : IN Species : Ne, Xe.

[767] V. Schmidt. Double photoionization near threshold. AIPCP, 205:241-5, 1990. Exp - Keys : MI - Species : He.

[768 H. Schwier, S. Jetzke, R. Hippler and H.O. Lutz. Continuum transitions in multiphoton-ionization and electron scattering. MP, p. 45-57, 1988. Exp. review - Keys : MP - General.

[769] J.H Scofield. Angular and polarization correlations in photoionization and radiative recombination. PRA, 40:3054-60, 1989. Theory - Keys : OU - Species : $\mathrm{Ni}^{26+}, \mathrm{Ba}^{46+}$. 
[770] J.H. Scofield. Angular distribution of photoelectrons from polarized X-rays. PS, 41:59-62, 1990. Theory Keys : IN - General.

[771] P. Scott. Photoionization of heavy atoms. ADW2, p. 90-7, 1989. Th. review - Keys : OU - Species : Zn, Rb, $\mathrm{Cd}, \mathrm{Cs}, \mathrm{Ba}, \mathrm{Hg}$.

[772] M.J. Seaton. Outer-region contributions to radiative transition probabilities. JPB, 19:2601-10, 1986. Theory Keys : OU - General.

[773] J.M. Seminario and F.C. Sanders. Application of $Z$-dependent theory to autoionizing states of Helium-like atoms : Feshbach projection method. PRA, 42:2562-72, 1990. Theory-Keys : AI - Species : He seq with $2 \leq Z \leq 10\left(\mathrm{He}, \mathrm{Li}^{+}, \mathrm{Be}^{2+}, \mathrm{B}^{3+}, \mathrm{C}^{4+}, \mathrm{N}^{5+}, \mathrm{O}^{6+}, \mathrm{F}^{7+}, \mathrm{Ne}^{8+}\right)$.

[774] J.M.P. Serrao. Absorption spectrum of magnesium. JQSRT, 41:307, 1989. Theory - Keys : OU - Species : Mg.

[775] R. Shakeshaft. Theory of multiphoton ionization of atoms by intense laser fields. JOSAB, 4:705-19, 1987. Th. review - Keys : MP - General.

[776] R. Shakeshaft. Ejection of high-energy photoelectrons by intense laser light. ZPD, 14:271-2, 1989. Theory Keys : MP - General.

[777] R. Shakeshaft and R.M. Potvliege. Approximate theory for multiphoton ionization of an atom by an intense field. PRA, 36:5478-81, 1987. Theory - Keys : MP - General.

[778] N. Shanthi. Photoionisation from the 3p subshell of zinc. JPB, 21:L427-31, 1988. Theory-Keys : IN - Species : $\mathrm{Zn}$.

[779] N. Shanthi and P.C. Deshmukh. Near-threshold structure in the photoionization of krypton 3p. PRA, 37:1773-4, 1988. Theory - Keys : IN - Species : Kr.

[780] N. Shanthi, P.C. Deshmukh and S.T. Manson. Photoionization cross-section, angular-distribution and spinpolarization calculations for the $3 p$ and $3 d$ subshell of krypton. PRA, 37:4720-9, 1988. Theory - Keys : IN Species : Kr.

[781] N. Shanthi and P.C. Deshmukh. Xenon 4p photoionization near the 4d Cooper minimum : interchannel coupling effects. PRA, 40:2400-3, 1989. Theory - Keys : IN - Species : Xe.

[782] N. Shanthi and P.C. Deshmukh. Low- and intermediate-energy photoionisation of palladium. JPB, 23:61-76, 1990. Theory - Keys : ou - Species : Pd.

[783] A. Sharma, G.L. Bhale and M.A.N. Razvi. Three-photon resonant ionization in atomic potassium via S, P, D, F series of Rydberg states. Pramana, 35:95-104, 1990. Exp-Keys : FP - Species : K.

[784] D.A. Shirley, U. Becker, P.A. Heimann and B. Langer. Near threshold behaviour of photoelectron satellite intensities. JPCo, 48-C9:427-38, 1987. Exp. review - Keys : IN - Species : He, Ne, Ar.

[785] H. Shoranzer, M. Vildberger, K.H. Schartner, B. Mobus and B. Magel. Fluorescence studies on the photoionization cross sections of the Kr 4s electrons near threshold. PLA, 150:281-5, 1990. Exp-Keys : OU - Species : $\mathrm{Kr}$.

[786] V.A. Shurygin. Approximating photoionization cross sections for subshells of certain elements in the energy range $130-1500 \mathrm{eV}$. SAE, 63:641-4, 1988. Th. review - Keys : OU IN-General.

[787] L. Smentek-Mielezarek and B.A. Hess. Third order ab initio calculation of amplitudes of two-photon absorption for ions across the lanthanide series. JCP , 90:1390-5, 1988. Theory-Keys : FP -Species : $\mathrm{Pr}^{3+}, \mathrm{Nd}^{3+}, \mathbf{E u}^{3+}$, $\mathrm{Gd}^{3+}, \mathrm{Tb}^{3+}, \mathrm{Tm}^{3+}$.

[788] H. Smid and J.E. Hansen. Can a bound Rydberg level be pushed into its own continuum ?. JPB, 20:6541-51, 1987. Theory - Keys : AI - Species : Xe.

[789| Z. Smit, M. Kregar and D. Glavic-Cindro. Double photoionization of $K$ - and $L$-shell electron pairs. PRA, 40:6303-7, 1989. Theory - Keys : IN MI - Species : He, Ne.

[790] S.J. Smith, J.H. Eberly and J.W. Gallagher. Multiphoton bibliography: 1987-88. NBS-LP92, Suppl. 6 (University of Rochester). Review - Keys : MP - General. 
[791] S.J. Smith and G. Leuchs. Angular correlations in multiphoton ionization of atoms. AAMP, 24:157-223, 1987 Review - Keys : FP MP AI - Species : Na, Cs, Ba.

[792] K. Sommer, M.A. Baig, W.R.S. Garton and J. Hormes. The high resolution subvalence p-shell absorption spectrum of potassium I. PS, 35:637-44, 1987. Exp-Keys : OU - Species : K.

[793] B. Sonntag. A survey on the experimental developments in atomic photoionization. JPCo, 48-C9:439-51, 1987 Exp. review - Keys : OU - Species : He, Li, Ne, Ar, U, Ca ions.

[794] S.L. Sorensen, R. Carr, S.J. Schaphorst, S.B. Whitfield and B. Crasemann. Coster-Kronig yields in silve: measured with synchrotron radiation. PRA, 39:6241-6, 1989. Exp-Keys : IN - Species : Ag.

[795] J.K. Spong, A. Imamoglu, J.D. Kmetec and S.E. Harris. Extreme-ultraviolet fluorescence from core-excitec levels of neutral rubidium. OL, 14:168-70, 1988. Exp-Keys : AI - Species : Rb.

[796] A.F. Starace. Electron correlation effects in nonresonant multiphoton ionization processes. PS, T17:221-7, 1987 Th. review - Keys : MP - Species : Ar, Xe.

[797\} A.F. Starace. The calculation of photoionization cross sections. ADW2, p. 1-6, 1988. Th. review - Keys : OU General.

[798] J.E. Stewart, H.C. Bryant, P.G. Harris, A.H. Mobagheghi, J.B. Donahue, C.R. Quick, R.A. Reeder, V. Yuan C.R. Hummer, W.W. Smith and S. Cohen. Effects of electric fields on the photodetachement cross section o: the $\mathrm{H}^{-}$ion near threshold. PRA, 38:5628-38, 1988. Exp-Keys : SF - Species : $\mathrm{H}^{-}$.

[799] R.C. Stoneman, D.S. Thomson and T.F. Gallagher. Microwave multiphoton transitions between Rydberg state! of potassium. PRA, 37:1527-40, 1988. Exp - Keys : MP - Species : K.

[800] R. Stringat, G. Fabre and M. Taleb. Two-photon resonant processes in atomic barium vapour. JPB, 21:4107-16 1988. Exp - Keys : FP - Species : Ba.

[801] Q. Su and J.H. Eberly. Stabilization of a model atom in superintense field ionisation. JOSAB, 7:564-8, 1990 Theory - Keys : MP - General.

[802] V.I. Sukhorukov, A.N. Hopersky, I.D. Petrov, V.A. Yavna and V.F. Demekhin. Double photoexcitation processes at the near K-edge region of $\mathrm{Ne}, \mathrm{Na}$ and $\mathrm{Ar}$. JP, 48:1677-83, 1987. Theory-Keys : IN AI - Species: Ne, Na Ar.

[803] B. Sundaram and L. Armstrong Jr. Role of excited states in multiphoton dynamics. PRA, 38:152-61, 1988 Theory - Keys : MP - Species : H.

[804] B. Sundaram and L. Armstrong Jr. Modeling strong-field above-threshold ionization. JOSAB, 7:414-24, 1990 Theory - Keys : MP - General.

[805] S.M. Suuskind, S.C. Cowley and E.J. Valeo. Multiphoton ionisation in a short-range potential : a nonpertur bative approach. PRA, 42:3090-106, 1990. Theory - Keys : MP - General.

[806] T. Suzuki and T. Kasuya. Optogalvanic study of photodetachment of $\mathrm{O}^{-}$near threshold. PRA, 36:2129-33 1987. Exp - Keys : oU - Species : $\mathrm{O}^{-}$.

[807] S. Svensson, N. Muller, N. Böwering, U. Heinzmann, W. Wijesundera and V. Radojevic. Angle and spin resolver photoelectron spectroscopy of the 4 subshell in atomic Ytterbium. JPB, 21:L179-85, 1988. Exp \& theory Keys : $\mathrm{QU}$ - Species : Yb.

[808] A. Szoke and C.K. Rhodes. Theoretical model of inner-shell excitation by outer-shell electrons. PRL, 56:720-3 1986. Theory - Keys : MP OU IN - Species : Xe.

[809] C.Y. Tang, P.G. Harris, A.H. Mohagheghi, H.C. Bryant, C.R. Quick, J.B. Donahue, R.A. Reeder, S. Cohen W.W. Smith and J.E. Stewart. Observation of multiphoton detachment of the $\mathrm{H}^{-}$ion. PRA, 39:6068-71, 1989 Exp-Keys : MP - Species : $\mathrm{H}^{-}$.

[810] X. Tang, T.N. Chang, P. Lambropoulos, S. Fournier and L.F. DiMauro. Multiphoton ionization of magnesiun with configuration interaction calculations. PRA, 41:5265-8, 1990. Theory - Keys : MP - Species : Mg.

[811] X. Tang, A. L'Huillier, P. Lambropoulos, M.H.R. Hutchinson and K.M.M. Ness. Comment on "laser-inducer continuum structure in xenon". PRL, 62:111-12, 1988. Exp \& theory-Keys : MP - Species : Xe. 
[812] X. Tang, A. Lyras and P. Lambropoulos. The many faces of resonances in multiphoton ionization. JOSAB, 7:456-62, 1990. Theory - Keys : AI MP - General.

[813] X. Tang, H. Rudolph and P. Lampropoulos. Nonperturbative approach to atomic multiphoton processes under intense, short laser pulses. PRL, 65:3269-2, 1990. Theory - Keys : MP - Species : H.

[814] S.S. Tayal. Autoionizing states in photoionization of the $3 s^{2} 3 p^{4}{ }^{3}$ ground state of sulfur. PRA, 38:729-36, 1988. Theory - Keys : OU AI - Species: S.

[815] K.T. Taylor, M.H. Nayteh and C.W. Clark. Atomic spectra and collisions in external fields. Plenum Press, 1988. Review - Keys : MP - General.

[816] A. Temkin and A.K. Bhatia. Polarization asymmetry in two-electron photodetachment : a cogent test of the ionization threshold law. PRA, 38:494-6, 1988. Theory - Keys : MI - Species : $\mathrm{Te}^{-}$.

[817] C.E. Theodosiou. Photoionization of the $\mathrm{N} 2 \mathrm{p}^{2} 3 \mathrm{p}^{2} \mathrm{~S}_{1 / 2}$ state. PRA, 37:1795-7, 1988. Theory - Keys : oU Species : N.

[818] J.S. Thompson, D.J. Pegg, R.N. Compton and G.D. Alton. Photodetachment of He-: angular distributions of photoelectrons. AIPCP, 191:770-2, 1990. Exp-Keys: OU - Species: $\mathrm{He}^{-}$.

[819] J.S. Thompson, D.J. Pegg, R.N. Compton and G.D. Alton. Angular distributions of electrons from the photodetachment of metastable $\mathrm{He}^{-}$. JPB, 23:L15-19, 1990. Exp-Keys : OU - Species : $\mathrm{He}^{-}$.

[820] X.M. Tong, J.M. Li and R.H. Pratt. Delayed maxima in the photoionization cross sections of atomic ions. PRA, 42:5348-51, 1990. Theory - Keys : OU - Species : Fe ions, Xe ions.

[821] R. Trainham, G.D. Fletcher, N.B. Mansour and D.J. Larson. Photodetachment threshold shift in a strong laser field. PRL, 59:2291-4, 1987. Exp-Keys : MP - Species : $\mathrm{Cl}^{-}$.

[822] E. Trefftz. Aluminium photoionisation cross sections. JPB, 21:1761-76, 1988. Theory - Keys : OU - Species : Al.

[823] J. Tremblay, M. Larzilliere, F. Combet-Farnoux and P. Morin. Photoelectron spectroscopy of atomic iodine produced by laser photodissociation. PRA, 38:3804-7, 1988. Exp \& theory - Keys : OU - Species : I.

[824] M. Trippenbach, K. Rzazewski and R. Grobe. Angular distribution of photoelectrons in above-threshold ionization of atomic hydrogen. SJQE, 18:727-9, 1988. Theory - Keys : MP - Species : H.

[825] M. Trippenbach, K. Rzazewski and R. Grobe. Angular distribution of photoelectrons in the above-threshold ionization of atomic hydrogen. PRA, 37:4194-200, 1988. Theory - Keys : MP - Species : H.

[826] M. Trippenbach, K. Rzazewski, M.V. Fedorov and A.E. Kazakov. Semiclassical matrix elements, essential state models and perturbation theory of above threshold ionisation. JPB, 22:1193, 1989. Theory - Keys : MP Species : H.

[827] F. Trombetta and S. Basile. Light scattering and multiphoton ionisation in the strong-field high-frequency regime. JPB, 22:L553-7, 1989. Theory - Keys : MP - Species : H.

[828] F. Trombetta, S. Basile and G. Ferrante. Multiphoton one-step ionization of Helium at 1064 nm. JMO, 36:891907, 1989. Theory - Keys : MP - Species : He.

[829] F. Trombetta, S. Basile and G. Ferrante. Nonperturbative treatments of nonresonant multiphoton ionization of the hydrogen atom : weak-field limit. JOSAB, 6:554-9, 1989. Theory - Keys : FP - Species : H.

[830] F. Trombetta, S. Basile and G. Ferrante. Strong-field high-frequency approximation to the multiphoton ionization of hydrogen. JOSAB, 7:598-606, 1990. Theory - Keys: MP - Species : H.

[831] F. Trombetta, G. Ferrante and S. Basile. Asymmetric angular distributions in multiphoton ionisation of helium by elliptically polarised light. JPB, 21:L539-44, 1988. Theory - Keys : MP - Species : He.

[832] J. Tulkki. Multiple excitation at xenon 5s photoionization threshold. PRL, 62:2817-20, 1989. Theory - Keys : ou - Species : Xe.

[833] J. Tulkki, T. Åberg, S.B. Whitfield and B. Crasemann. Quantum approach to photoelectron recapture in post-collision interaction. PRA, 41:181-5, 1990. Theory-Keys : IN-Species : Ar. 
[834] J.A. Tully, M. Le Dourneuf and C.J. Zeippen. Photoionisation of the $\mathrm{B}^{+}\left({ }^{1} \mathrm{~S}^{\mathrm{e}}\right)$ ground state. AA, 211:485-94, 1989. Theory - Keys : OU AI - Species : $\mathrm{B}^{+}$.

[835] J.A. Tully, M.J. Seaton and K.A. Berrington. Atomic data for opacity calculations: XIV. The beryllium sequence. JPB, 23:3811-3837, 1990. Theory - Keys : OU - Species : Be seq with $4 \leq Z \leq 26\left(\mathrm{Be}, \mathrm{B}^{+}, \mathrm{B}^{+}, \mathrm{C}^{2+}, \mathrm{N}^{3+}, \mathrm{O}^{4+}\right.$, $\left.\mathrm{F}^{5+}, \mathrm{Ne}^{6+}, \mathrm{Na}^{7+}, \mathrm{Mg}^{8+}, \mathrm{Al}^{9+}, \mathrm{Si}^{10+}, \mathrm{S}^{12+}, \mathrm{Ar}^{14+}, \mathrm{Ca}^{16+}, \mathrm{Fe}^{22+}\right)$.

[836] J.A. Tully, M.J. Seaton and K.A. Berrington. An update of $f$ values and photoionization cross sections for the Be isoelectronic sequence. JPCo, this issue, 1990. Theory - Keys : ou - Species : Be seq.

[837] K. Ueda, K. Ito, Y. Sato and T. Namioka. High-resolution absorption spectrum of atomic calcium in the vicinity of the $4 \mathrm{p}_{1 / 2,3 / 2}^{2}$ thresholds. PS, 41:75-7, 1990. Exp-Keys : OU - Species : Ca.

[838] K. Veda, K. Maeda, K. Ito and T. Namioka. High resolution measurement and quantum defect analysis of the $8 s^{\prime}$ and $6 d^{\prime}$ autoionisation resonances of Krypton. JPB, 22:L481-5, 1989. Exp \& theory - Keys : AI - Species : $\mathrm{Kr}$.

[839] K. Unnikrishnan. Time-dependent $S$-matrix theory of multiphoton ionization : crucial role of the adiabatic hypothesis. JPB, 23:L541-5, 1990. Theory - Keys : MP - General.

[840] S. Vacquie, A. Gleizes and M. Sabsabi. Measurement of the photodetachment cross-section of the negative ion of fluorine. PRA, 35:1615-20, 1987. Exp-Keys : OU - Species : $\mathrm{F}^{-}$.

[841] N. Vaeck and J.E. Hansen. Calculations for $1 \mathrm{~s}^{2} 3 / 3 l^{\prime}$ states in $\mathrm{C}^{2+}, \mathrm{N}^{3+}, \mathrm{O}^{4+}, \mathrm{Ne}^{6+}$ and $\mathrm{Xe}^{50+}$. JPB, 22:3137-53, 1989. Theory-Keys : A I-Species: $\mathrm{C}^{2+}, \mathrm{N}^{3+}, \mathrm{O}^{4+}, \mathrm{Ne}^{6+}, \mathrm{Xe}^{60+}$.

[842] G. van der Laan and B.T. Thole. Angular dependent photoelectric yield. Measurement of the La 3d Auger and autoionization lifetime widths. JESRP, 46:123-9, 1988. Exp-Keys : IN AI - Species : La.

[843] W.J. van der Meer, P. van der Meulen, M. Volmer and C.A. De Lange. Absolute He I $\alpha$ photoionisation cross sections of $\mathrm{O}$ atoms and electronically excited $\mathrm{O}_{2}\left({ }^{1} \Delta_{g}\right)$. CP, 126:385-93, 1988. Exp - Keys : oU - Species : O.

[844] W.J. van der Meer, M. Volmer and C.A. De Lange. Absolute photoionisation cross-sections of atomic and molecular fluorine at $584 \AA$. JESRP, 49:263-72, 1989. Exp-Keys : oU - Species : F.

[845] P. van der Meulen, C.A. de Lange, M.O. Krause and D.C. Mancini. A photoelectron spectroscopic study of oxygen atoms near the ${ }^{4} \mathrm{P}^{e}$ threshold using synchrotron radiation. PS, 41:837-40, 1990. Exp - Keys : OU Species: $\mathrm{O}$.

[846] P. van der Straten, R. Morgenstern and A. Niehaus. Angular dependent post-collision interaction in Auger processes. ZPD, 8:35-45, 1988. Theory - Keys : IN - Species : Ar, Xe.

[847] W. van der Water, S. Yoakum, T. van Leeuwen, B.E. Suer, L. Moorman, E.J. Galvez, D.R. Mariani and P.M. Koch. Microwave multiphoton ionization and excitation of helium Rydberg atoms. PRA, 42:572-91, 1990. Exp $\&$ theory - Keys : MP - Species : He.

[848] H.B. van Linden van der Heuvell and H.G. Muller. Limiting cases of excess-photon ionization. MP, p. 25-34, 1988. Exp. review - Keys : MP - Species : Xe.

[849] L.D. Van Woerkom and W.E. Cooke. Complementary branching ratios by satellite excitation. PRL, 57:1711-14, 1986. Theory - Keys : IN - Species : Ba.

[850] L.D, van Woerkom and W.E. Cooke. Energy-dependent branching ratios of autoionizing states. PRA, 37:332637, 1988. Exp-Keys : AI - Species : Ba.

[851] L.D. van Woerkom, R.R. Freeman, W.E. Cooke and T.J. McIlrath. Saturation effects in the spatial and energy distributions in short pulse high intensity multiphoton ionisation. JMO, 36:817-27, 1989. Theory - Keys : MP - General.

[852] R.D. Verma and A. Chanda. A new technique to study Rydberg states by multiphoton ionization spectroscopy. JPCo, 48-C7:683-5, 1987. Exp-Keys : MP - Species : Ba.

[853] E.P. Vidolova-Angelova, D.A. Angelov, S.T. Mincheva, V.N. Fedoseev, V.I. Mishin and A.D. Zuzikov. Tm autoionization Rydberg states in the vinicity of the third and fourth atomic ionization limit. ZPD, 13:115-21, 1989. Exp-Keys : AI - Species : Tm. 
[854] F.P. Vidolova-Angelova, L.N. Ivanov and D.A. Angelov. Autoionisation decay of highly excited Rydberg Tm states. JPB, 21:3877-90, 1988. Theory - Keys : AI -- Species : Tm.

[855] A.B. Voitkiv and V.A. Parzdzersky. Threshold photodecay of a negative ion in the presence of a low-lying autoionising state. JPB, 19:1633-43, 1986. Theory - Keys : OU - General.

[856] E. von Raven, M. Meyer, M. Pahler and B. Sonntag. Electron-electron co ncidence studies of the decay of photoexcited rare gas core resonances. JESRP, 52:677-88, 1990. Exp-Keys : IN - Species : Ar, Kr, Xe.

[857] A. Wada, Y. Adachi and C. Hirose. Observation of autoionization spectrum of $\mathrm{Kr} 7 \mathrm{~d}^{\prime}-5 \mathrm{p}^{\prime}$ and $9 \mathrm{~s}^{\prime}-5 \mathrm{p}^{\prime}$ visible transitions by optogalvanic spectroscopy. JPCh, 90:6645-8, 1986. Exp - Keys : AI - Species : Kr.

[858] A. Wague. Calculation of the photoelectron angular distribution asymmetry parameter $\beta_{2 \mathrm{p}}$ near the (3s3p) ${ }^{1} \mathrm{P}$ resonance of $\mathrm{He}^{*}$. ZPD, 8:251-4, 1988. Theory - Keys : OU AI - Species : He.

[859] A. Wague. Intermediate-coupling effects on the $n=2$ resonant photo-ionization of multiply-charged heliumlike ions. ZPD, 13:123-8, 1989. Theory-Keys : AI - Species : He seq with $6 \leq Z \leq 26\left(\mathrm{C}^{4+}, \mathrm{O}^{6+}, \mathrm{Ne}^{8+}\right.$, $\left.\mathrm{Mg}^{10+}, \mathrm{Fe}^{24+}\right)$.

[860] A. Wague. Resonance photoionisation of the metastable $2^{1,3} \mathrm{~S}$ helium near the $n=2$ and $n=3$ excitation thresholds of He. ZPD, 15:199-202, 1990. Theory-Keys : OU AI - Species : He.

[861] K. Wang and S.I. Chu. Dynamics of multiphoton excitation and quantum diffusion in Rydberg atoms. PRA, 39:1800-8, 1989. Theory - Keys : MP - Species : H.

[862] L. Wang, J.W. Haus and K. Rzazewski. Angular photoelectron distributions in above-threshold ionization with bichromatic excitation. PRA, 42:6784-93, 1990. Theory - Keys : MP - General.

[863] Q. Wang and C.H. Greene. Quantal description of diamagnetic quasi-Landau-resonances. PRA, 40:742-51, 1989. Theory - Keys : SF - Species : H.

[864] W. Weber, B. Breuckmann, R. Huster, W. Menzel, W. Mehlhorn, M.H. Chen and K.G. Dyall. Very strong correlation effects in Auger spectra through the collapse of d orbitals : the 2p Auger spectrum of free Ca atoms. JESRP, 47:105-30, 1988. Exp - Keys : IN - Species : Ca.

[865] G.R. Welch, M.M. Kash, L. Hsu and D. Kleppner. Positive-energy structure of the diamagnetic Rydberg spectrum. PRL, 62:1975-8, 1989. Theory-Keys : SF - Species : Li.

[866] G. Wendin. Giant resonances as a probe of collective excitations in atoms and solids. NATO2, p. 171-211, 1987. Th. review - Keys : OU IN - General.

[867] G. Wendin, L. Jonsson and A. L'Huillier. Many electron dynamics of heavy atoms in intense laser fields : effects of screening and correlation. JOSAB, 4:833-45, 1987. Th. review - Keys : MP - Species : Xe.

[868] U. Werner and W. Jitschin. L-vacancy decay in heavy elements $(72 \leq Z \leq 82)$ by the synchrotron photoionization method. PRA, 38:4009-18, 1988. Exp-Keys : IN - Species : Hf, Ta, W, Re, Os, Ir, Pt, Au, Hg, Tl, Pb.

[869] S.B. Whitfeld, G.B. Armen, J.C. Levin and B. Crasemann. Vacancy multiplication following Ni L-shell photoionization. PRA, 37:419-25, 1988. Exp-Keys : IN MI-Species : Ni.

[870] W. Wijesundera and H.P. Kelly. Correlation satellites in the photoelectron spectrum of argon. PRA, 39:634-43, 1989. Theory - Keys : oU - Species : Ar.

[871] A.A. Wills, A.A. Cafolla, F.J. Currell, J. Comer, A. Svensson and M.A. MacDonald. A photoelectron study of resonance structure in the argon correlation satellites. JPB, 22:3217-26, 1989. Exp - Keys : OU - Species : Ar.

[872] A.A. Wills, A.A. Cafolla and J. Comer. Resonance structure in the 5p, 5s and satellite photoelectron lines of xenon. JPB, 23:2029-36, 1990. Exp-Keys : DU - Species : Xe.

[873 A.A. Wills, A.A. Cafolla, A. Svensson and J. Comer. Resonance structure in the neon photoelectron sattelites. JPB, 23:2013-28, 1990. Exp-Keys : OU - Species : Ne.

[874] B.G. Wilson, C. Iglesias and F. Rogers. Near-threshold photoabsorption cross sections for nonhydrogenic ions. PRA, 38:4633-8, 1988. Theory - Keys : OU - General.

[875] P. Winkler. A unified many-body theory of dielectronic recombination and autoionization. IJQCQCS, 19:201-16, 1986. Th. review - Keys : AI - General. 
[876] D. Wintgen and H. Friedrich. Photoabsorption calculations for the magnetised hydrogen atom in the quasiLandau region. JPB, 19:L99-104, 1986. Theory - Keys : SF - Species : H.

[877] B. Wolff, H. Rottke, D. Feldmann and K.H. Welge. Multiphoton-ionization of hydrogen atoms in intense laser fields. ZPD, 10:35-43, 1988. Exp - Keys : MP - Species : H.

[878] H.Y. Wong, A.R.P. Rau and C.H. Greene. Negative-ion photodetachment in an electric field. PRA, 37:2393-403, 1988. Theory - Keys : SF-Species : $\mathrm{S}^{-}$.

[879] B.R. Wu, S. Zhang, J.W. Zhong, J.Z. Qiu, S.F. Hu, J. Lu and J.Z. Sun. Measurement of the (5 $\mathrm{p}_{3 / 2}$ ss) 1 and $(5 \mathrm{p} 3 / 2 n \mathrm{~d})_{3}$ autoionizing levels of strontium by the multistep excitation technique. ChP, 8:71-7, 1988. Exp Keys : AI - Species : Sr.

[880] I.J. Wu and H. Xie. Theoretical calculation of two-photon excitation of $\mathrm{Hg}$ atom. APS, 35:556-60, 1986. Theory - Keys : FP - Species: Hg.

[881] L. Wu and J. Xi. The autoionisation widths of He I below the $N=3 \mathrm{He}^{+}$threshold. JPB, 23:727-35, 1990. Theory - Keys : AI - Species : He.

[882] Y.J. Wu and J.M. Li. Non-relativistic self-consistent-field calculation of two-photon transitions in atomic inner shells for Xe. JPB, 21:1509-1517, 1988. Theory-Keys : FP IN - Species : Xe.

[883] Y.J. Wu and J.M. Li. Correlation interaction in atomic inner shell photoelectric effect. APS, 38:1056-61, 1989. Theory - Keys : IN - Species : $\mathrm{Ne}^{+}, \mathrm{Ne}$.

[884] F.J. Wuilleumier, D.L. Ederer and J.L. Picqué. Photoionization and collisional ionization of excited atoms using synchrotron and laser radiations. AAMP, 23:198-286, 1987. Review - Keys : DU IN AI - Species : He, Li, Ne, $\mathrm{Na}, \mathrm{K}, \mathrm{Xe}, \mathrm{Cs}, \mathrm{Ba}$.

[885] X. Xing, D. Charalambidis and C. Fotakis. Influence of space charge on photoelectron angular distributions and energy spectra in resonance multiphoton ionization. OC, 79:181-6, 1990. Exp-Keys : MP - Species : Xe.

[886] J.Q. Xu. New interpretation of the Ag L-series X-ray spectrum. ZPD, 13:25-7, 1989. Exp \& theory - Keys : IN - Species : Ag.

[887] A. Yagishita, S. Aksela, T. Prescher, M. Meyer, M. Richter, E. von Raven and B. Sonntag. Decay channels of the $4 p$ resonances in atomic Sr. JPB, 21:945-53, 1988. Exp-Keys: IN AI - Species : Sr.

[888] K. Yamada and H. Takahashi. Optical anisotropy induced by two intense laser fields in sodium vapor. OC, 75:307-10, 1990. Exp - Keys : MP - Species : Na.

[889] D. Yao and M.H. Nayfeh. Photoionization spectrum of $\mathrm{H}$ in strong DC electric fields. PRA, 35:2508-17, 1987. Theory - Keys : SF - Species: $\mathrm{H}$.

[890] D. Yao and M.H. Nayfeh. $m=1$ photoionization spectrum of hydrogen in strong DC electric fields. PRA, 36:4072-5, 1987. Exp-Keys : SF - Species : H.

[891] G.H. Yao and Z.Z. Xu. Coherent effects in intense laser-field induced autoionization. APS, 37:1760-6, 1988. Exp - Keys : AI MP - General.

[892] G.H. Yao and Z.Z. Xu. Peak-switching of the photoelectron spectrum. APS, 38:864-8, 1989. Exp - Keys : MP - General.

[893] G.H. Yao and Z.Z. Xu. Ejection of energetic photoelectrons in laser-induced autoionisation : effects of high-order ionisation processes. JPB, 22:211-21, 1989. Theory - Keys : AI MP - General.

[894] G.H. Yao, W. Yu, Z.Z. Xu and R.Q. Chen. Above-threshold ionization by a smooth laser pulse. APS, 39:35-9, 1990. Theory - Keys : MP - General.

[895] T.S. Yih, H.H. Wu, H.T. Chan, C.C. Chu and B.J. Pong. Measurement of the photoabsorption cross-section of magnesium vapor in the VUV range. ChJP, 27:136-9, 1989. Exp-Keys : OU - Species : Mg.

[896] K. Yoshino, A.S.C. Cheung, J.R. Esmond, W.H. Parkinson, D.E. Freeman, S.L. Guberman, A. Jenouvrier, B. Coquart and M.F. Merienne. Improved absorption cross-sections of oxygen in the wavelength region 205-240 nm of the Herzberg continuum. PSI, 36:1469-75, 1988. Exp - Keys : OU - Species : O. 
[897] W. Yu and Z.Z. Xu. Model study for the time evolution of above-threshold ionization. JPB, 22:L427-31, 1989. Theory - Keys : MP - General.

[898] Y. Yu and M.J. Seaton. Atomic data for opacity calculations. IV. Photoionization cross sections for C Il. JPB, 20:6409-29, 1987. Theory - Keys : OU - Species : $\mathrm{C}^{+}$.

[899] S.A. Zapryagaev, B.A. Zon and Y.A. Nefedov. Photoionization of doubly excited atomic states. OS, 63:422-6, 1988. Theory - Keys : OU - Species : Mg, Ca, Sr.

[900] J. Zhang and T.A. King. Photoionisation in the TI anti-Stokes Raman laser. JPB, 23:1587-94, 1990. Exp Keys : oU - Species : Tl.

[901] Y. Zhu, R.R. Jones, W. Sandner, T.F. Gallagher, P. Camus, P. Pillet and J. Boulmer. Multiphoton double ionisation of $\mathrm{Ba}$ from 280 to $700 \mathrm{~nm}$. JPB, 22:585-99, 1989. Exp - Keys : MP MI - Species : Ba.

[902] Y. Zhu, E.Y. Xu and T.F. Gallagher. Energy and angular distributions of autoionization electrons and channel interaction effects for the Sr 5pns $J=1$ autoionizing series. PRA, 36:3751-67, 1987. Exp - Keys : OU AI Species : Sr.

[903] V.A. Zilitis. Photoionization cross sections for ions of the potassium isoelectronic series calculated by the DiracFock method. OS, 65:588-90, 1988. Theory-Keys : oU - Species : K seq with $20 \leq Z \leq 28\left(\mathrm{Ca}^{+}, \mathrm{Sc}^{2+}, \mathrm{Ti}^{3+}\right.$, $\left.\mathrm{Cr}^{5+}, \mathrm{Fe}^{7+}, \mathrm{Ni}^{9+}\right)$.

[904] P. Zimmerer, N. Grun and W. Scheid. Trajectory method for time-dependent Thomas-Fermi equations and application to Ar atoms in laser fields. PLA, 134A:57-61, 1988. Theory-Keys : MP - Species : Ar.

1905] P. Zimmermann. Study of multicharged photoions using synchrotron radiation. CAMP, 23:45-53, 1989. Review - Keys : IN MI - Species : La, Ce, Gd, Dy.

[906] M. Zubek and G.C. King. Photoionisation studies of autoionising states of helium between 69 and 77 eV. JPB, 22:3411-21, 1989. Exp - Keys : AI - Species : He.

[907] M. Zubek, G.C. King, P.M. Rutter, and F.H. Read. Threshold photoionisation studies in helium and nitrogen. SPIG86, -0-:111-13, 1986. Exp - Keys : ou - Species : He, N.

[908] A.D. Zyurikov, V.S. Letokhov, V.I. Mishin, and V.N. Fedoseev. Laser resonance photoionization spectroscopy of excited and autoionization atomic states of rare-earth elements. I. Europium. OS, 63:572-8, 1988. Exp Keys : OU AI - Species : Eu.

[909] A.D. Zyurikov, V.I. Mishin, and V.N. Fedoseev. Laser resonance photoionization spectroscopy of excited and autoionization atomic states of rare-earths. III. Neodynium. OS, 64:287-8, 1988. Exp-Keys : OU AI - Species : Nd.

\section{INDEX BY SPECIES, TYPES OF PROCESSES AND RELATED}

The index of the bibliography is made by species, grouped into isoelectronic or isonuclear sequences, by the keys defining the processes (multiple keys being used as in the previous paragraph to emphasize several aspects of the work or some ambiguity in the classification : inner valence shells being sometimes simultaneously classified in OU and $\mathrm{IN}$ ) and by the type of publication ( $T$ for a paper describing a calculation, $E$ for an experiment, $M$ for $a$ 'mixture' or experimental and theoretical analysis, $R$ for a review). Finally, it should be noticed that most of the papers relevant to the $\mathrm{H}$ sequence are general papers, concerning the development of new approaches, tested on $\mathrm{H}$ rather than on $\mathrm{a}$ model problem.

\section{Reviews, general studies}

OU 023T 030R 059T 109T 159E 185T 202R 206R 210T 229R 253T 304T 366T 408T 451T 465T 492T 538R 561R 562R 564R 583E 606T 695E 696T 697T 755R 772T 786R 797R 855T 866R 874T

IN 059T 176T 228R 229R 318T 320T 342E 389T 437T 442T 443T 495T 612T 628R 639R 770T 786R 866R

AI 106 $138 \mathrm{~T}$ 139T 147T 189T 203T 303T 334T 366T 495T 518R 530T 535T 550T 705T 812T 875T 891E 893E

MI 130R 131T 147T 216T 518R 628R 
SF $\quad 122 \mathrm{~T}$ 230T 705T

FP 207T 231T 335T 430E 530T 649T 694E

MP O06R 013T 036T 073T 075T 082T 083R 094T 096R 111T 122T 123T 130R 131T 133R 138T 139T 147T 149T 171T 172T 187R 188T 196T 197T 205E 216T 217T 219R 220T 224T 254R 255R 267T 271T 276T 287T 290T 298T 299R 300R 301T 302T 306T 307T 308T 309R 310T 311T 318T 334T 340E 342E 345T 348T 351T 362T 364T 365T 372T 414T 415T 416T 418T 442T 443T 444T 467T 469R 483T 490T 496E 510T 511T 517R 518R 519R 525R 532R 535T 588T 595T 596R 603E 607T 615R 617T 647T 648T 653T 673T 679T 680T 681R 693T 707T 709T 711T 712T 719T 726T 754T 768R 775R 776T 777T 790R 801T 804T 805T 812T 815R 839T 851T 862T 891E 892E 893E 894T 897T

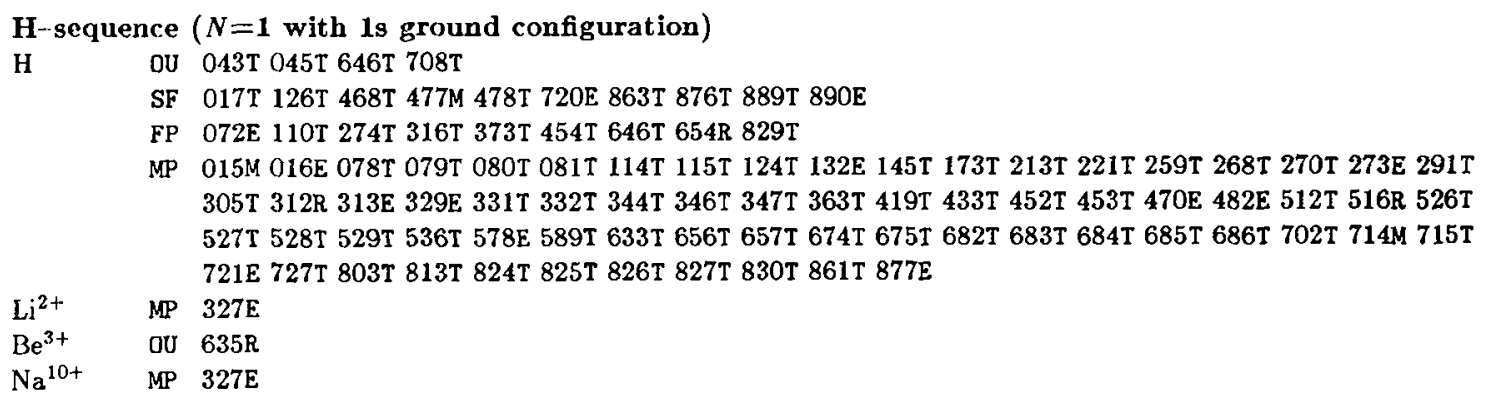

He-sequence ( $N=2$ with $18^{2}$ ground configuration)

Many $Z$ OU 315T $(Z=2-14,16,18-20,24-26,28,36)$ $756 \mathrm{~T}(Z=2-10,12-14,16,18,20,26)$

IN $097 \mathrm{E}(Z=16,18,19,21-24,26,36)$

$571 \mathrm{~T}$

AI $101 \mathrm{~T} 349 \mathrm{R} 571 \mathrm{~T}$

183T $(Z=1,2,6,7,8)$

449T $(Z=10,12,18,20,22,24,26,28)$

$552 \mathrm{~T}(Z=2-6,8)$

$773 \mathrm{~T}(Z=2-10)$

$859 \mathrm{~T}(Z=6,8,10,12,26)$

MI $146 \mathrm{~T}$

MP $146 \mathrm{~T}$

$\mathrm{H}^{-} \quad$ OU 044T 143T 354T 424T 634T 729T

AI $141 \mathrm{~T} 183 \mathrm{~T}$ 324T 378E 393T 629T 630T

SF 194E 277T 278T 279T 280T 296T 297T 353T 704T 798E

FP $046 \mathrm{~T} 227 \mathrm{~T}$

MP $\quad 095 \mathrm{~T}$ 223T 226T 269T 286T 289T 336T 609T 611T 809E

He OU O47E 068E 142T 323T 371T 386T 387E 458R 466E 531T 708T 728T 740T 749T 793R 858T 860T 884R $907 \mathrm{E}$

IN $591 \mathrm{~T} 784 \mathrm{R} 789 \mathrm{~T} 884 \mathrm{R}$

AI $\quad 107 \mathrm{~T} 183 \mathrm{~T}$ 212T 323T 324T 338T 368E 371T 386T 392T 394E 457T 479E 551T 552T 566T 597T 629T 631T $637 \mathrm{~T} 773 \mathrm{~T} 858 \mathrm{~T}$ 860T 881T 884R 906E

MI $056 \mathrm{~T} 466 \mathrm{E}$ 480E 509E 767E $789 \mathrm{~T}$

FP $056 \mathrm{~T} 368 \mathrm{E} 456 \mathrm{E}$

MP 077E 134E 193T 330T 475E 493T 502T 543R 616E 828T 831T 847M

$\mathrm{Li}^{+} \quad$ OU $144 \mathrm{~T} 395 \mathrm{~T} 531 \mathrm{~T} 749 \mathrm{~T}$

AI $551 \mathrm{~T} 552 \mathrm{~T} 629 \mathrm{~T} 773 \mathrm{~T}$

$\mathrm{Be}^{2+} \quad$ OU $635 \mathrm{R}$

AI $551 \mathrm{~T} 552 \mathrm{~T} 773 \mathrm{~T}$

$\mathrm{B}^{3+} \quad$ OU $635 \mathrm{R}$

AI 551T 552T 773T

$\mathrm{C}^{4+} \quad$ OU $410 \mathrm{E} 635 \mathrm{R}$

IN $409 \mathrm{E} 411 \mathrm{E}$

AI $183 \mathrm{~T} 552 \mathrm{~T} 566 \mathrm{~T}$ 773T $859 \mathrm{~T}$

$\mathrm{N}^{5+} \quad$ AI $\quad 183 \mathrm{~T} 565 \mathrm{~T}$ 566T 650T 651M 773T 


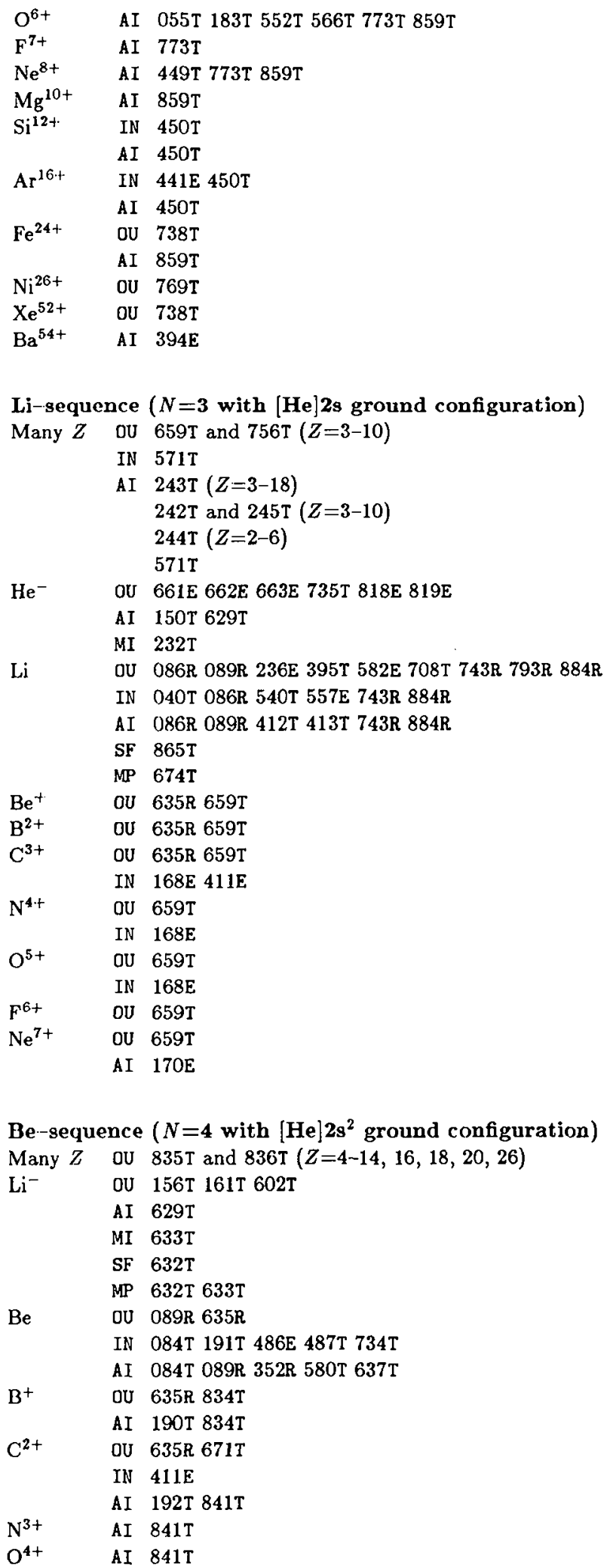

Be-sequence ( $N=4$ with [He $] 2 \mathrm{~s}^{2}$ ground configuration)

Many $Z$ OU $835 \mathrm{~T}$ and $836 \mathrm{~T}(Z=4-14,16,18,20,26)$

$\mathrm{Li}^{-} \quad$ OU $156 \mathrm{~T} 161 \mathrm{~T}$ 602T

AI $629 \mathrm{~T}$

MI $633 \mathrm{~T}$

SF $632 \mathrm{~T}$

MP $632 \mathrm{~T}$ 633T

$\mathrm{Be} \quad$ OU $089 \mathrm{R} 635 \mathrm{R}$

IN 084T 191T 486E 487T 734T

AI $084 \mathrm{~T}$ 089R 352R 580T $637 \mathrm{~T}$

$\mathrm{B}^{+} \quad$ OU $635 \mathrm{R} 834 \mathrm{~T}$

AI 190T 834T

$\mathrm{C}^{2+} \quad$ OU $635 \mathrm{R} 671 \mathrm{~T}$

IN $411 \mathrm{E}$

AI 192T $841 \mathrm{~T}$

$\mathrm{N}^{3+} \quad$ AI $\quad 841 \mathrm{~T}$

$\mathrm{O}^{4+}$ AI $841 \mathrm{~T}$ 


$\begin{array}{lll}\mathrm{Ne}^{6+} & \text { AI } & 841 \mathrm{~T} \\ & \text { IN } & 627 \mathrm{~T} \\ \mathrm{Ar}^{14+} & \text { AI } & 112 \mathrm{E} \\ \mathrm{Ti}^{18+} & \text { AI } & 112 \mathrm{E} \\ \mathrm{Xe}^{50+} & \text { AI } & 841 \mathrm{~T}\end{array}$

B-sequence ( $N=5$ with [He] $2 \mathrm{~s}^{2} 2 \mathrm{p}$ ground configuration)

Many $Z$ IN 179 T $(Z=6-10,12,14,16,18,20,22,26,28,36,42,47,54)$

B IN $191 \mathrm{~T}$

$\mathrm{C}^{+} \quad$ OU $898 \mathrm{~T}$

C-sequence ( $N=6$ with $[\mathrm{He}] 2 \mathrm{~s}^{2} 2 \mathrm{p}^{2}$ ground configuration)

Many $Z$ OU $175 T(Z=6-26)$

$545 \mathrm{~T}$ and $687 \mathrm{~T}(Z=6-14,16,18,20,26)$

$\mathrm{B}^{-} \quad$ OU $661 \mathrm{E}$

C IN 411 E 645 R

$\mathrm{O}^{2+} \quad$ OU $546 \mathrm{~T}$

$\mathrm{Ne}^{4+} \quad$ AI $448 \mathrm{~T}$

$\mathrm{Xe}^{48+}$ OU $175 \mathrm{~T}$

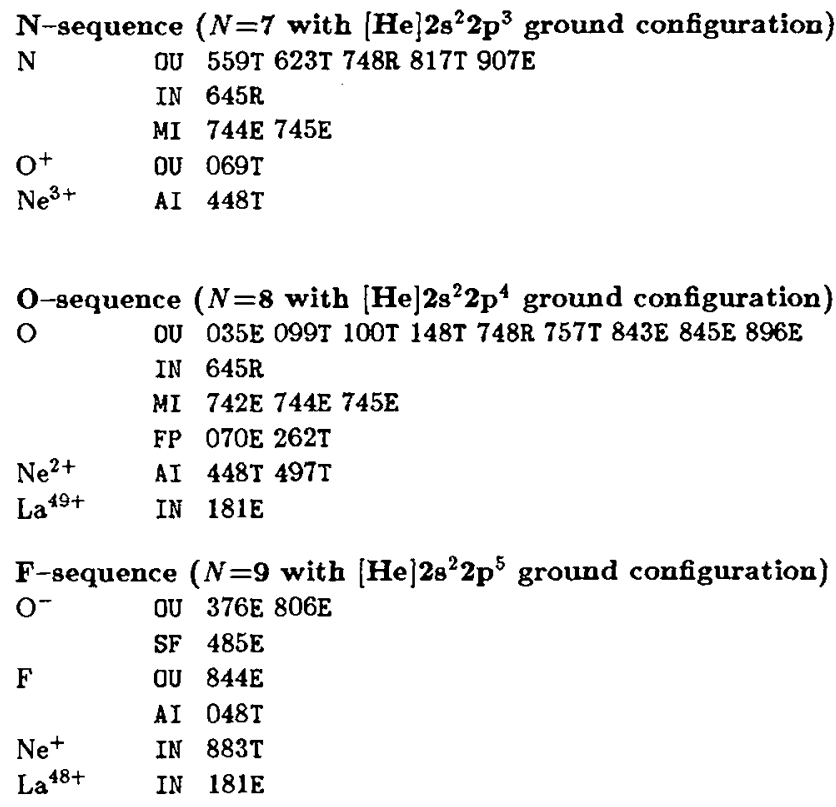

Ne-sequence ( $N=10$ with $[\mathrm{He}] 2 \mathrm{~s}^{2} 2 \mathrm{p}^{6}$ ground configuration)

Many $Z$ AI $178 \mathrm{~T}$

$\mathrm{F}^{-} \quad$ OU $500 \mathrm{E} 840 \mathrm{E}$

FP 119E 225R 652T

MP 218T 222T 343T 500E 523E

$\mathrm{Ne} \quad$ OU O86R 089R O92E 152E 387E 406M 743R 748R 763E 793R 873E 884R

IN O07R 010E 011E 033T 086R 092E 160T 195T 250T 544T 645R 743R 766R 784R 789T 802T 883T 884R

AI 086R 089R 700T 743R 802T 884R

MI 742E 744E 745E 789T

MP 475E $616 \mathrm{E}$

$\mathrm{Mg}^{2+}$ IN $627 \mathrm{~T}$ 
$\begin{array}{lll}\mathrm{Ar}^{8+} & \text { IN } & 627 \mathrm{~T} \\ \mathrm{Ba}^{46+} & \text { OU } & 769 \mathrm{~T}\end{array}$

Na-sequence ( $N=11$ with $[\mathrm{Ne}] 3 \mathrm{~s}$ ground configuration)

Many $Z$ IN $177 \mathrm{~T}(Z=18,22,26)$

AI $178 \mathrm{~T}$

$\mathrm{Na} \quad$ OU $067 \mathrm{~T}$ 162E 233R 236E 581E 608T 736T 737T 884R

IN 234E 235E 246T 247T 258E 672T 716E 802T 884R

AI $108 \mathrm{~T} 162 \mathrm{E} 672 \mathrm{~T} 791 \mathrm{R} 802 \mathrm{~T} 884 \mathrm{R}$

FP O20T 067T 233R 234E 339E 484E 791R

MP O38E 326E 791R 888E

$\mathrm{Mg}^{+} \quad$ AI $317 \mathrm{E}$

$\mathrm{Al}^{2+} \quad$ AI $317 \mathrm{E} 605 \mathrm{~T}$

$\mathrm{Si}^{3+} \quad$ AI $605 \mathrm{~T}$

$\mathrm{Se}^{23+} \quad$ OU $636 \mathrm{E}$

$\mathrm{Mg}$-sequence ( $N=12$ with $[\mathrm{Ne}] 3 \mathrm{~s}^{2}$ ground configuration)

$\mathrm{Na}^{-}$OU $029 \mathrm{~T}$

AI $322 \mathrm{~T}$

MP $343 \mathrm{~T}$

$\mathrm{Mg} \quad$ OU 021T 165T 166T 398E 598T 599T 601T 774T 895E $899 \mathrm{~T}$

IN $084 \mathrm{~T} 380 \mathrm{E} 627 \mathrm{~T} 645 \mathrm{R}$

AI 021T 084T 166T 239E 352R 637T

FP $600 T$

MP 240E 397E 462E 810T

$\mathrm{Al}^{+} \quad$ oU $256 \mathrm{~T}$

$\mathrm{Ar}^{6+}$ IN $627 \mathrm{~T}$

Al-sequence ( $N=13$ with [Ne] $3 s^{2} 3 p$ ground configuration)

Al

ou $822 \mathrm{~T}$

IN $049 \mathrm{E} 645 \mathrm{R}$

AI $548 \mathrm{~T}$

MI $049 E$

MP $548 \mathrm{~T}$

Si-sequence ( $N=14$ with [Ne] $3 s^{2} 3 p^{2}$ ground configuration)

Many $Z$ OU 687T $(Z=14,16,18,20,26)$

$\mathrm{Si} \quad$ OU $356 \mathrm{~T} 579 \mathrm{~T}$

IN $645 \mathrm{R}$

P-sequence $\left(N=15\right.$ with $[\mathrm{Ne}] 3 \mathrm{~s}^{2} 3 \mathrm{p}^{3}$ ground configuration)

$\mathrm{Si}^{-} \quad$ OU $029 \mathrm{~T}$

$\mathrm{P} \quad \mathrm{DU} \quad 579 \mathrm{~T}$

IN 701E

$\mathrm{S}$-sequence ( $N=16$ with $[\mathrm{Ne}] 3 \mathrm{~s}^{2} 3 \mathrm{p}^{4}$ ground configuration)

$\mathrm{S}$ OU $579 \mathrm{~T} 814 \mathrm{~T}$

IN $645 \mathrm{R}$ 701E

AI $321 \mathrm{~T} 814 \mathrm{~T}$

Cl-sequence ( $N=17$ with $[\mathrm{Ne}] 3 \mathrm{~s}^{2} 3 \mathrm{p}^{5}$ ground configuration)

$\mathrm{S}^{-} \quad$ SF $878 \mathrm{~T}$

FP 563E 
Ar-sequence ( $N=18$ with $[\mathrm{Ne}] 3 \mathrm{~s}^{2} 3 \mathrm{p}^{6}$ ground configuration)

$\mathrm{Cl}^{-} \quad$ FP $093 \mathrm{~T}$ 121E 272T 420T

MP $076 \mathrm{E}$ 218T 222T 343T 522E 523E $821 \mathrm{E}$

$\mathrm{Ar} \quad$ OU $024 \mathrm{R}$ 086R 088E 089R 092E 369E 577E 730T 743R 746E 747E 748R 760E 762R 793R 870T 871E

IN OO7R 010E 033T 086R 092E 158E 184T 208T 209T 250T 263E 285E 295E 367E 369E 381E 388E 435T 524T 539M 627T 641T 645R 724T 733T 743R 753T 784R 802T 833T 846T 856E

AI $086 \mathrm{R}$ 089R 352R 743R 760E 762R 802T

MI 130R 184T 263E 508E

FP $573 \mathrm{E} 652 \mathrm{~T} 664 \mathrm{M}$

MP 130R 174E 330T 391E 473E 534E 543R 667M 713R 796R 904T

$\mathrm{K}^{+} \quad$ OU $560 \mathrm{~T} 626 \mathrm{~T}$

AI $214 \mathrm{~T}$

K-sequence ( $N=19$ with [Ar] 4 s neutral ground configuration)

Many $Z$ OU $903 \mathrm{~T}(Z=20,21,22,24,26,28)$

$\begin{array}{lll}\text { K } & \text { AI } & 725 \mathrm{E} \\ & \text { OU } & 560 \mathrm{~T} \text { 731T } 792 \mathrm{E} 884 \mathrm{R}\end{array}$

IN $403 \mathrm{E} 701 \mathrm{E} 884 \mathrm{R}$

AI $884 \mathrm{R}$

FP 403E 783E

MP $799 \mathrm{E}$

$\mathrm{Ca}^{+}$OU 587T

$\mathrm{Fe}^{7+}$ oU $756 \mathrm{~T}$

Ca-sequence ( $N=20$ with $\left[A \mathbf{A r} \mid \mathbf{4 s}^{2}\right.$ neutral ground configuration)

$\mathrm{K}^{-} \quad \mathrm{MI} \quad 058 \mathrm{~T}$

$\mathrm{Ca}$ OU O54E 360E 458R 463T 464T 584E 837E 899T

IN 084T $182 \mathrm{~T}$ 204E 586M 645R 688E 701E 864E

AI 084T 352R 355T 360E 463T 464T 521E 586M 604E 637T 692E

SF $692 \mathrm{E}$

FP $018 M 703 R$

MP $461 \mathrm{E}$

$\mathrm{Fe}^{6+} \quad$ oU $756 \mathrm{~T}$

Sc-sequence ( $N=21$ with $[\mathrm{Ar}] 4 \mathrm{~s}^{2} 3 \mathrm{~d}$ neutral ground configuration)

$\mathrm{Ca}^{-}$OU $662 \mathrm{E}$

AI $377 E$

Se OU 333R

IN $585 \mathrm{R} 765 \mathrm{R}$

MI 765 R

Ti-sequence ( $N=22$ with $[A \mathbf{r}] 4 \mathrm{~s}^{2} 3 \mathrm{~d}^{2}$ neutral ground configuration)

$\mathrm{Ti}$ IN $585 \mathrm{R} 658 \mathrm{E} 765 \mathrm{R}$

MI $765 \mathrm{R}$

V-sequence ( $N=23$ with $[A r] 4 s^{2} 3 d^{3}$ neutral ground configuration)

$\mathrm{V} \quad \mathrm{IN} 585 \mathrm{R}$

FP 750E 


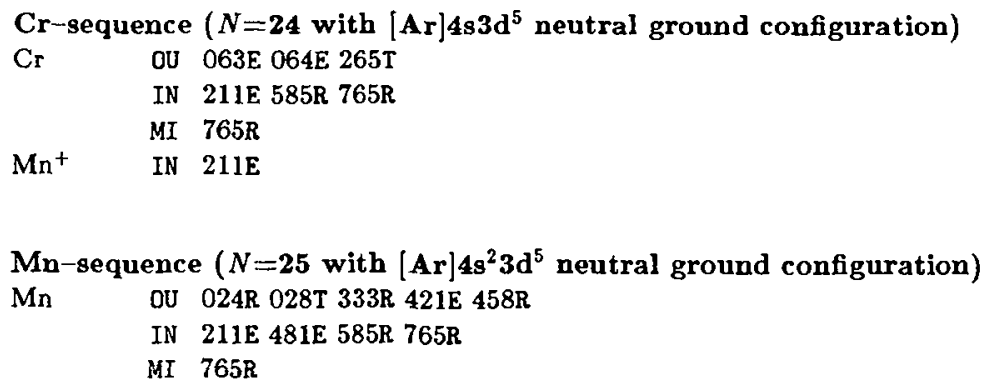

Ge-sequence ( $N=32$ with $[A r] 48^{2} 3 d^{10} 4 p^{2}$ neutral ground configuration) $\mathrm{Ge}$ AI $352 \mathrm{R}$

Se-sequence ( $N=34$ with $[A r] 4 s^{2} 3 \mathrm{~d}^{10} 4 \mathrm{p}^{4}$ neutral ground configuration)

$\mathrm{Se}$ OU $432 \mathrm{E}$

IN $432 E$ 


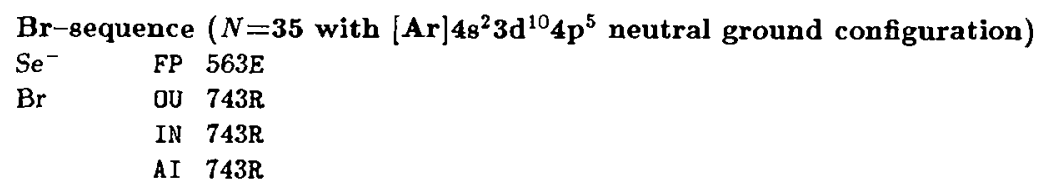

Kr-sequence ( $N=36$ with $\{\mathrm{Ar}\} 4 \mathrm{~s}^{2} 3 \mathrm{~d}^{10} 4 \mathrm{p}^{6}$ neutral ground configuration)

$\mathrm{Br}^{-} \quad$ FP $119 \mathrm{E}$

MP $120 \mathrm{M} 218 \mathrm{~T} 222 \mathrm{~T}$

$\mathrm{Kr} \quad$ OU O89R 370E 553E 743R 747E 748R 785E

IN 007R 009T 010E 032T 157E 158E 294E 370E 383E 384E 388E 434T 435T 436T 447E 592T 620E 640E 641] 665E 722T 743R 779T 780T 856E

AI 089R 252E 352R 592T 743R 838E 857E

MI 130R 382E 620E

FP 252E 573E 590E

MP O77E 130R 135R 136T 391E 473E 474E 520E 666E 667M

$\mathrm{Rb}$-sequence ( $N=37$ with $[\mathrm{Kr}] \mathbf{5 s}$ neutral ground configuration)

$\mathrm{Rb} \quad$ OU $771 \mathrm{R}$

IN O08E 012M 472E

AI 407E 795E

MI $472 E$

MP 014T

Sr--sequence $\left(N=38\right.$ with $[\mathrm{Kr}] 58^{2}$ neutral ground configuration)

$\mathrm{Rb}^{-} \quad \mathrm{SF} \quad 357 \mathrm{~T}$

$\mathrm{Sr} \quad$ OU $052 \mathrm{~T}$ 053T 899T 902E

IN $472 \mathrm{E}$ 641T $887 \mathrm{E}$

AI 241T 402E 476E 515E 676T 692E 879E 887E 902E

MI 153M 472E 501R

SF $692 \mathrm{E}$

FP $379 \mathrm{E} 515 \mathrm{E} 655 \mathrm{E}$

MP 153M 501R 669R

Y-sequence ( $N=39$ with $[\mathrm{Kr}] 5 \mathrm{~s}^{2} 4 \mathrm{~d}$ neutral ground configuration)

$\mathrm{Y}$ OU 333R

IN $423 E$

Mo-sequence ( $N=42$ with $[\mathrm{Kr}] 5 \mathrm{~s} 4 \mathrm{~d}^{5}$ neutral ground configuration)

Mo IN 610T 678T

FP 610 T

Pd-sequence ( $N=46$ with $[\mathrm{Kr}] 4 \mathrm{~d}^{10}$ neutral ground configuration)

$\mathrm{Pd} \quad \mathrm{OU}$ 782T

IN $007 \mathrm{R} 678 \mathrm{~T}$

Ag-sequence ( $N=47$ with $[\mathrm{Kr}] 5 \mathrm{s4d} \mathrm{d}^{10}$ neutral ground configuration)

$\mathrm{Ag}$ OU O01E 037E 198R

IN O07R 455E 568E 610T 794E 886M

AI $061 \mathrm{E}$

MI O01E

FP 061E 610T 
Cd-sequence ( $N=48$ with $[\mathrm{Kr}] 5^{2} \mathrm{~s}^{2} \mathrm{~d}^{10}$ neutral ground configuration)

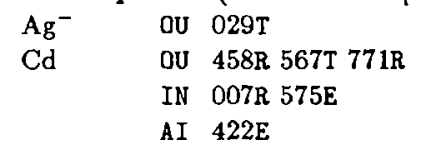

In-sequence $\left(N=49\right.$ with $[K r] 5 s^{2} 4 d^{10} 5 p$ neutral ground configuration)

In OU O62E $619 \mathrm{E}$

IN $568 \mathrm{E}$

AI 062E 488E 619E

Sn-sequence ( $N=50$ with $[K r] 5 s^{2} 4 d^{10} 5 p^{2}$ neutral ground configuration)

Sn AI 352R

Sb-sequence ( $N=51$ with $[\mathrm{Kr}] 5 \mathrm{~s}^{2} 4 \mathrm{~d}^{10} 5 \mathrm{p}^{3}$ neutral ground configuration)

$\mathrm{Sb}$ IN $572 \mathrm{E}$

Te-sequence ( $N=52$ with $[\mathrm{Kr}] 5 \mathrm{~s}^{2} 4 \mathrm{~d}^{10} 5 \mathrm{p}^{4}$ neutral ground configuration)

$\mathrm{Te}$ IN $431 \mathrm{E} 568 \mathrm{E}$

I-sequence ( $N=53$ with $[\mathrm{Kr}] 5 \mathrm{~s}^{2} 4 \mathrm{~d}^{10} 5 \mathrm{p}^{5}$ neutral ground configuration)

$\mathrm{Te}^{-} \quad$ MI $816 \mathrm{~T}$

I OU $823 \mathrm{M}$

IN 200T $625 \mathrm{E}$

Xe-sequence ( $N=54$ with $[\mathrm{Kr}] 5 \mathrm{~s}^{2} 4 \mathrm{~d}^{10} 5 \mathrm{p}^{6}$ neutral ground configuration)

$\mathrm{I}^{-}$OU 024R 029T

FP 119E 506T

MP 218T 222T

Xe OU 024R 027T 057R 090E 199R 201R 370E 458R 499T 514T 638E 747E 748R 761E 808T 832T 872E 884R

IN O10E 022T O27T 032T 039T 041T 042E 087E 090E 091E 127E 158E 199R 370E 383E 385E 388E 434T 435T 436T 445E 446E 471E 593T 610T 622E 641T 642T 643M 644E 722T 766R 781T 808T 846T 856E 882T 884R

AI O42E 352R 516R 788T 884R

MI 027T 130R 382E 501R 504T 505T 516R 537R 614M 689E

FP 066M 417T 489E 504T 505T 573E 590E 610T 882T

MP O03E 004E 005R 077E 118R 130R 135R 136T 137E 169E 213T 288T 319R 361T 391E 404E 405E 473E 474E 494T 501R 503E 516R 537R 666E 667M 668E 669R 670R 710T 713R 796R 808T 811T 848R 867R 885E

$\mathrm{Cs}^{+} \quad$ OU $199 \mathrm{R}$

$\begin{array}{lll} & \text { IN } & 199 \mathrm{R} \\ \mathrm{Ba}^{2+} & \text { OU } & 199 \mathrm{R} 375 \mathrm{E}\end{array}$

IN 199R 722T

$\mathrm{La}^{3+} \quad$ OU O26T 199R 375E

IN $199 \mathrm{R}$

Cs-sequence ( $N=55$ with [Xe]6s neutral ground configuration)

Many $Z$ OU 050T 513T $(Z=55-65)$

Cs OU 024R 025T 050T 057R 237E 251T 264T 333R 341T 624T 732T 771R 884R

IN $568 \mathrm{E} 576 \mathrm{E}$ 622E $884 \mathrm{R}$

AI $791 \mathrm{R} 884 \mathrm{R}$

MI $621 \mathrm{E}$

FP 399E 549E 791R

MP 791R

$\mathrm{Ba}^{+} \quad$ OU $050 \mathrm{~T} 547 \mathrm{R}$ 


$\begin{array}{rll} & \text { IN } & 722 \mathrm{~T} \\ & \text { AI } & 350 \mathrm{E} \\ \mathrm{La}^{2+} & \text { OU } & \text { 025T 026T 050T } \\ & \text { IN } & 284 \mathrm{E}\end{array}$

Ba-sequence ( $N=56$ with [Xe]6s ${ }^{2}$ neutral ground configuration)

$\mathrm{Ba}$ OU 027T 051T 074T 117E 233R 333R 337E 358T 374E 390E 428E 440E 498T 717E 752E 771R 884R

IN 027T 085R 116E 542T 568E 622E 698T 699T 722T 849T 884R

AI 102M 103E 104E 105E 113T 154E 163E 238E 248E 249E 293E 325R 352R 425E 426E 427E 428E 676T 677T 691E 751R 752E 791R 850E 884R

MI O27T 328E 429E 621E 901E

SF $427 \mathrm{E} 751 \mathrm{R}$

FP 233R 293E 440E 459E 655E 791R 800E

MP 292E 328E 427E 429E 791R 852E 901E

$\mathrm{La}^{+} \quad$ OU $026 \mathrm{~T}$

IN $284 \mathrm{E}$

$\mathrm{Ce}^{2+} \quad$ IN $284 \mathrm{E}$

$\mathrm{Pr}^{3+} \quad$ FP $787 \mathrm{~T}$

La-sequence ( $N=57$ with [Xe]6s ${ }^{2} 5 \mathrm{~d}$ neutral ground configuration)

La OU 024R 026T 374E 717E

IN 282T 585R 842E 905R

AI $842 \mathrm{E}$

MI $905 \mathrm{R}$

$\mathrm{Ce}^{+} \quad$ IN $284 \mathrm{E}$

$\mathrm{Pr}^{2+}$ IN 284E

$\mathrm{Nd}^{3+} \quad$ FP $787 \mathrm{~T}$

Ce-sequence ( $N=58$ with $[\mathrm{Xe}] 6 \mathrm{~s}^{2} 4 \mathrm{f5d}$ neutral ground configuration)

$\mathrm{Ce}$ OU 374E 717E

IN 042E 281E 282T 905R

AI $042 E$

MI 281E 905R

$\mathrm{Pr}^{+} \quad$ IN $284 \mathrm{E}$

$\mathrm{Nd}^{2+} \quad$ IN $284 \mathrm{E}$

Pr-sequence ( $N=59$ with $[\mathrm{Xe}] 6 \mathrm{~s}^{2} 4 \mathrm{f}^{\beta}$ neutral ground configuration)

Pr IN 282T

$\mathrm{Nd}^{+} \quad$ IN $284 \mathrm{E}$

Nd-sequence ( $N=60$ with $[\mathrm{Xe}] 6 \mathrm{~s}^{2} 4 \mathrm{f}^{4}$ neutral ground configuration)

Nd OU $909 E$

IN $282 \mathrm{~T}$

AI $909 \mathrm{E}$

$\mathrm{Sm}^{2+} \quad$ IN $284 \mathrm{E}$

$\mathrm{Eu}^{3+} \quad$ FP $787 \mathrm{~T}$

Pm-sequence ( $N=61$ with $[\mathrm{Xe}] 6 \mathrm{~s}^{2} 4 \mathrm{f}^{6}$ neutral ground configuration)

$\mathrm{Sm}^{+} \quad$ IN $284 \mathrm{E}$

$\mathrm{Eu}^{2+}$ IN $284 \mathrm{E}$

$\mathrm{Gd}^{3+} \quad$ FP $787 \mathrm{~T}$ 


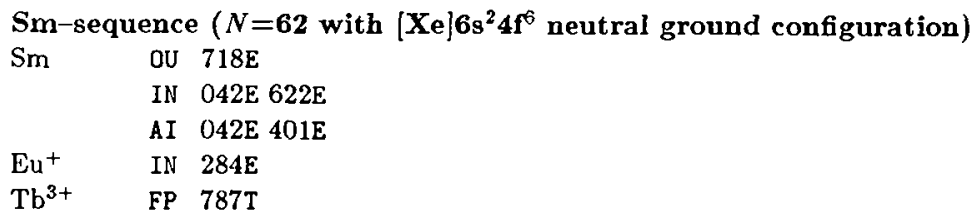

Eu-sequence ( $N=63$ with $[\mathrm{Xe}] 6 \mathrm{~s}^{2} 4 \mathrm{f}^{7}$ neutral ground configuration)

Eu OU 024R 031T 458R 718E 908E

IN $622 \mathrm{E}$

AI $908 \mathrm{E}$

Gd-sequence ( $N=64$ with $[\mathrm{Xe}] 6 \mathrm{~s}^{2} 4 \mathrm{f}^{7} 5 \mathrm{~d}$ neutral ground configuration)

Gd OU 201R 333R 594E 718E

IN O42E 282T 283E 905R

A I O42E 594E

MI $905 R$

FP $594 E$

Tb-sequence ( $N=65$ with $[\mathrm{Xe}] 6 \mathrm{~s}^{2} 4 \mathrm{f}^{9}$ neutral ground configuration)

Tb IN 281E 282T 283E

MI 281E

Dy-sequence ( $N=66$ with $[\mathrm{Xe}] 6 \mathrm{~s}^{2} 4 \mathrm{f}^{10}$ neutral ground configuration)

Dy IN 282T 283E 905R

MI $905 \mathrm{R}$

$\mathrm{Tm}^{3+} \quad$ FP $787 \mathrm{~T}$

Ho-sequence ( $N=64$ with [Xe] $6 s^{2} 4 f^{11}$ neutral ground configuration)

Ho IN $283 \mathrm{E} 678 \mathrm{~T}$

Er-sequence ( $N=68$ with $[X e] 6 s^{2} 4 f^{12}$ neutral ground configuration)

Er IN 042E 283E

AI $042 E$

Tm-sequence ( $N=69$ with $[X e] 6 s^{2} 4 f^{13}$ neutral ground configuration)

Tm IN 140E 275T 283E

AI $853 \mathrm{E} 854 \mathrm{~T}$

MI 275T

Yb-sequence ( $N=70$ with $[\mathrm{Xe}] 6 \mathrm{~s}^{2} 4 \mathrm{f}^{14}$ neutral ground configuration)

$\mathrm{Yb} \quad$ OU O86R 164T 460E 807E

IN O42E 086R 281E 283E 622E

AI $042 \mathrm{E} 086 \mathrm{R}$

MI $128 \mathrm{E} 281 \mathrm{E}$

FP 460E.

MP 128E

Hf-sequence ( $N=72$ with $[X e] 6 s^{2} 4 f^{14} 5 d^{2}$ neutral ground configuration)

Hf IN $868 \mathrm{E}$ 
Ta-sequence ( $N=73$ with $[\mathrm{Xe}] 6 \mathrm{~s}^{2} 4 \mathrm{f}^{14} 5 \mathrm{~d}^{3}$ neutral ground configuration)

Ta IN $868 \mathrm{E}$

W-sequence ( $N=74$ with $[\mathrm{Xe}] 6 \mathrm{~s}^{2} 4 \mathrm{f}^{14} 5 \mathrm{~d}^{4}$ neutral ground configuration) W IN $739 \mathrm{E} 868 \mathrm{E}$

Re-sequence ( $N=75$ with $[\mathrm{Xe}] 6 \mathrm{~s}^{2} 4 \mathrm{f}^{14} 5 \mathrm{~d}^{5}$ neutral ground configuration) Re IN 085R 868E

Os-sequence ( $N=76$ with $[\mathrm{Xe}] 6 \mathrm{~s}^{2} 4 \mathrm{f}^{14} 5 \mathrm{~d}^{6}$ neutral ground configuration) Os IN $868 \mathrm{E}$

Ir-sequence ( $N=77$ with $[\mathrm{Xe}] 6 \mathrm{~s}^{2} 4 \mathrm{f}^{14} 5 \mathrm{~d}^{7}$ neutral ground configuration) Ir IN $868 \mathrm{E}$

Pt-sequence ( $N=78$ with $[\mathrm{Xe}] 6 \mathrm{s4f^{14 }} 5 \mathrm{~d}^{9}$ neutral ground configuration) $\mathrm{Pt}$ IN $658 \mathrm{E} 868 \mathrm{E}$

Au-sequence ( $N=79$ with $[\mathrm{Xe}] 6 s 4 \mathrm{f}^{14} 5 \mathrm{~d}^{10}$ neutral ground configuration) Au OU O98E 198R

IN 507E 556E 658E 868E

FP 098E

Hg sequence ( $N=80$ with [Xe] $6 s^{2} 4 f^{14} 5 d^{10}$ neutral ground configuration)

$\mathrm{Hg}$ OU 396E 567T 574E 743R 758E 759E 771R

IN 261E 743R 759E $868 \mathrm{E}$

AI 186M 743R 759E

MI $690 \mathrm{E}$

FP $880 T$

Tl-sequence ( $N=81$ with $[\mathrm{Xe}] 6 \mathrm{~s}^{2} 4 \mathrm{f}^{14} 5 \mathrm{~d}^{10} 6 \mathrm{p}$ neutra] ground configuration)

$\mathrm{Tl}$ OU 129E 201R 333R 900E

IN $868 \mathrm{E}$

Pb--sequence ( $N=82$ with $[X e] 6 s^{2} 4 f^{14} 5 d^{10} 6 p^{2}$ neutral ground configuration)

$\mathrm{Pb}$ OU 201R 618E 743R

IN $438 \mathrm{E} 439 \mathrm{E}$ 556E 743R 868E

AI 488E 743R

MP 260E

At-sequence ( $N=85$ with $[\mathrm{Xe}] 6 \mathrm{~s}^{2} 4 \mathrm{f}^{14} 5 \mathrm{~d}^{10} 6 \mathrm{p}^{5}$ neutral ground configuration) At oU 314T

Rn-sequence ( $N=86$ with $[\mathrm{Xe}] 6 \mathrm{~s}^{2} 4 \mathrm{f}^{\mathrm{14}} 5 \mathrm{~d}^{10} 6 \mathrm{p}^{6}$ neutral ground configuration) Rn OU 057R 314T

Fr-sequence $(N=87$ with $[R n \mid 78$ neutral ground configuration)

Fr OU 314T 
Th-sequence ( $N=90$ with $[R n] 7 s^{2} 6 d^{2}$ neutral ground configuration)

Th OU 201R 333R

IN $556 \mathrm{E}$

U-sequence ( $N=92$ with $[R n] 7 s^{2} 5 f^{3} 6 d$ neutral ground configuration)

U OU O19E 793R

IN $439 \mathrm{E} 556 \mathrm{E}$

AI 558E 706E

FP O65E 359E 400E 554E 555E 558E

Isonuclear sequences

$\begin{array}{lll}\text { Many } Z & \text { IN } & 071 \mathrm{~T}(Z=01-26) \\ & & 257 \mathrm{R}(Z=44-99) \\ & 613 \mathrm{~T}(Z=02-36) \\ & & 645 \mathrm{R}(Z=06-08,10,12-14,16,18,20,26) \\ \mathrm{Ne} \text { ions } & \text { OU } & 266 \mathrm{~T} 491 \mathrm{~T} \\ \mathrm{Ar} \text { ions } & \text { OU } & 266 \mathrm{~T} 533 \mathrm{R} \\ \mathrm{Ca} \text { ions } & \text { oU } & 199 \mathrm{R} 793 \mathrm{R} \\ & \text { IN } & 199 \mathrm{R} \\ \mathrm{Fe} \text { ions } & \text { OU } & 199 \mathrm{R} 266 \mathrm{~T} \text { 541T } 820 \mathrm{~T} \\ & \text { IN } & 199 \mathrm{R} \\ \mathrm{Kr} \text { ions } & \text { OU } & 533 \mathrm{R} \\ \mathrm{Xe} \text { ions } & \text { OU } & 533 \mathrm{R} 820 \mathrm{~T} \\ \mathrm{U} \text { ions } & \text { OU } & 266 \mathrm{~T}\end{array}$

